\title{
LES FOUILLES RÉCENTES DU PECH DE L'AZÉ IV (DORDOGNE)
}

\author{
Alain TurQ ${ }^{1}$, Harold L. Dibble ${ }^{2}$, Paul GoldberG ${ }^{3}$, Shannon P. MCPHerron ${ }^{4}$, \\ Dennis SANDGATHE ${ }^{5}$, Heather JONES ${ }^{6}$, Kerry MAdDISON $^{7}$, Bruno MAUREILle $^{8}$, \\ Susan MENTZER ${ }^{9}$, Jack RINK ${ }^{10}$ et Alexandre STEENHUYSE ${ }^{11}$
}

\begin{abstract}
Mots-clés. Paléolithique moyen, Moustérien, Néandertal, micromorphologie, géoarchéologie, processus de formation des sites, feu, taphonomie, industrie lithique, restes humains.

Résumé. Le gisement moustérien du Pech de l'Azé IV se situe à Carsac (Dordogne) en Périgord, près de Sarlat, dans le sud-ouest de la France. Fouillé par F. Bordes durant les années 1970, il n'a jamais été véritablement publié si l'on excepte l'étude que nous avons consacrée en 2000 au matériel issu de ses fouilles. Les travaux de terrain effectués dans ce gisement entre 2000 et 2003 avaient pour but de faire une nouvelle lecture stratigraphique, de préciser les processus de formation du site et de dater la séquence. Nous présentons ici les premiers résultats concernant la géologie, ainsi qu'une description rapide des industries et les premières dates ESR, le programme engagé étant toujours en cours.

L'étude microscopique et macroscopique détaillée des huit principales couches stratigraphiques révèle que les dépôts sableux proviennent d'un même et vaste système de grottes englobant probablement l'ensemble des sites moustériens du Pech de l'Azé (I, II et IV). Le niveau inférieur, dépôt sombre et organique, est particulièrement intéressant. Il renferme de multiples surfaces de combustion souvent piétinées et peut-être des vidanges de foyers. Bien que déposés sous un climat tempéré, les sédiments d'origine anthropique sont bien conservés. $\mathrm{Si}$, dans la séquence, l'observation macroscopique a permis d’identifier des phénomènes de solifluxion, l'analyse microscopique n'a montré pratiquement aucun indice d'un climat froid. En ce qui concerne les industries, les nouvelles séries sont globalement comparables aux anciennes. Toutefois, quelques nouveaux éléments sont apparus, notamment la présence d'une technologie Quina dans la partie supérieure de la séquence.
\end{abstract}

Key-words. Middle Paleolithic, Mousterian, Neanderthal, micromorphology, geology, archaeology, site formation processes, lithic industries, human remains.

Abstract. The Mousterian site of Pech de l'Azé IV is located in Carsac (Dordogne) in the Perigord region of southwest of France, very near the town of Sarlat. The site was excavated during the 1970s by F. Bordes but it was never well published. In 2000 we published a study of the lithics from Bordes' excavations. From 2000 to 2003 we conducted new work at the site to re-study the stratigraphy in

1. Musée national de Préhistoire, F-24620 Les Eyzies. Courriel: alain.turq@culture.gouv.fr

2. Université de Pennsylvanie, 3451 Walnut Street, Philadelphie, PA 19104, USA. Courriel: hdibble@sas.upenn.edu

3. Département d'archéologie, Laboratoire de microstratigraphie, Université de Boston, 675 Commonwealth Ave., Suite 347, Boston, MA 02215, USA. Courriel : paulberg@bu.edu

4. Department of Human Evolution, Max Planck Institute for Evolutionary Anthropology, Deutscher Platz 6, D-04103 Leipzig, Germany. Courriel : mcpherron@eva.mpg.de

5. Department of Archaeology, Simon Fraser University, Burnaby, British Columbia, V3J-7N8, Canada.

6. School of Geography and Geology, McMaster University, USA.

7. Archaeometry and Geochronology (AGE) Laboratory, School of Geography \& Earth Sciences, McMaster University, Hamilton, Ontario, L8S 4K1, Canada. Courriel: kerry@chancellor-maddison.com

8. Pacea, Université de Bordeaux-I. Courriel: b.maureille@anthropologie.u-bordeaux1.fr

9. Department of Anthropology, University of Arizona, Tucson, AZ 85721-0030, USA. Courriel: smentzer@email.arizona.edu

10. School of Geography \& Earth Sciences, McMaster University, Hamilton, Ontario, L8S 4K1, Canada. Courriel: rinkwj@mcmaster.ca

11. Virginia Commonwealth University, School of world Studies, 312 N Shafer St., P.O. Box 842021, Richmond, Virginia 23284-2021, USA. 
order, to better understand the site's formation processes, and to obtain dates from the sequence. We present here some initial results on the geology, a description of the stone tool industries, and some initial ESR dates (the dating program is still in progress).

A detailed micro- and macroscopic study of the eight main stratigraphic levels shows that the sandy deposits come from a vast cave system that likely includes all the sites of Pech de l'Azé (I, II, and IV). The lowest level, which is characterized by dark, organic sediments, is particularly interesting. It includes within it multiple combustion zones that show evidence of having been subsequently trampled and perhaps emptied. Though the deposits come from a temperate climate, the sediments of anthropogenic origin are nevertheless well conserved. Macroscopically, there are traces of solifluction in the sequence, but the micromorphological analysis shows practically no evidence of a cold climate. As for the lithic industries, the new collection is in general comparable to that of Bordes. Nevertheless, there are some new elements including the presence of Quina technology in the upper part of the sequence.

Bien qu'étant l'une des archéo-séquences majeures du Périgord, le gisement du Pech de l'Azé IV est encore mal connu. C'est pour cette raison qu'il nous paraît important de présenter les premiers résultats avant même que l'ensemble des analyses ne soient achevées. Après une présentation détaillée du site (contexte géologique et historique des recherches), des méthodes de fouille mises en œuvre lors de notre intervention et des collections, nous nous intéresserons à la géologie du site, avant de donner les premiers éléments de chronologie numérique, puis une vue globale des industries lithiques, une approche des structures de combustion et enfin une étude des vestiges humains mis au jour. En guise de conclusion nous évoquerons les travaux en cours et leurs perspectives.

\section{PRÉSENTATION DU SITE CONTEXTE GÉOGRAPHIQUE ET GÉOLOGIQUE}

Le Pech de l'Azé IV appartient au complexe de sites du Paléolithique inférieur et moyen du Pech de l'Azé (fig. 1). Il est situé sur le flanc droit de la vallée partiellement sèche du ruisseau de Farge, tributaire de l'Enéa, affluent en rive droite de la Dordogne.

Le site est implanté au cœur du pays sarladais, entité géologique qui correspond à des formations sénoniennes détritiques accumulées dans une large dépression synclinale. Celle-ci, qui s'étire depuis Périgueux au nord/ nord-ouest jusqu'à Gourdon au sud/sud-est, a été recouverte à partir du Coniacien moyen, mais surtout durant le Santonien, par d'épaisses séries sableuses déposées dans un environnement de plage et d'estran (Platel, 1989). L'hétérogénéité lithologique de ces formations, qui peut conduire à trouver localement des faciès sableux non consolidés, est, au moins en partie, responsable du modelé spécifique actuel (Konik, 1999). Celui-ci se caractérise par

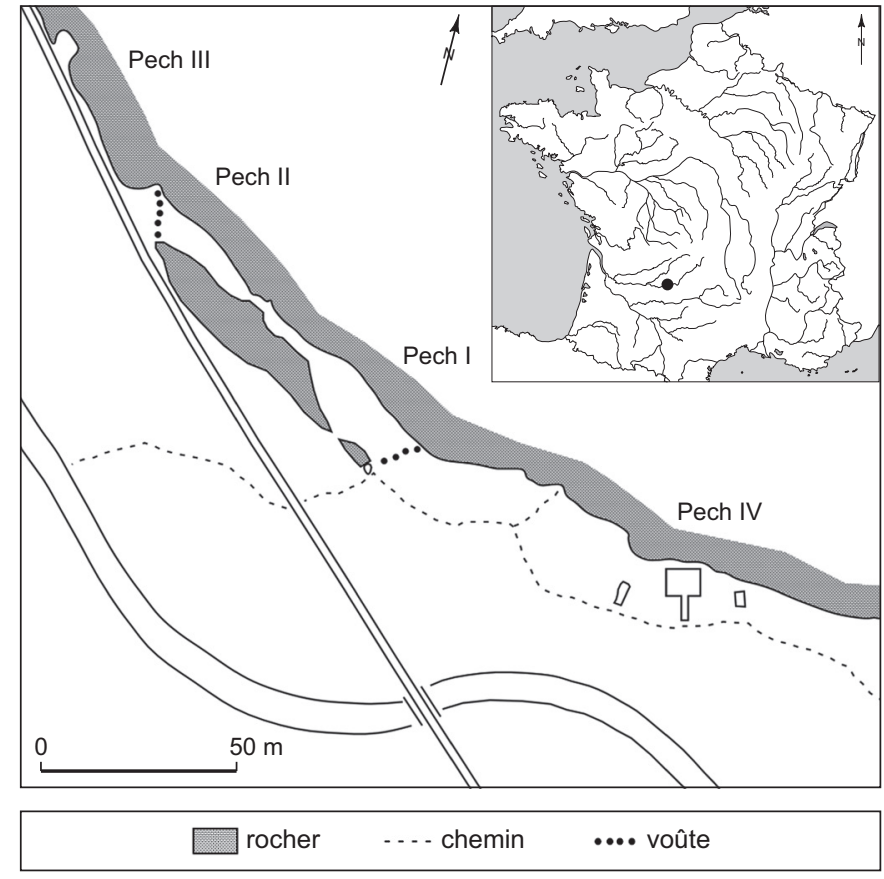

Fig. 1 - Pech de l'Azé à Carsac en Dordogne: localisation des différents gisements (DAO: S. McPherron, Max Planck Institute).

des buttes souvent couvertes de chênes verts, les «pechs» - dont l'étymologie est à rechercher dans le latin podium, signifiant point haut, hauteur - qui émergent dans des dépressions appelées "plaines», vouées à l'agriculture. Ce relief qui s'apparente à celui de certains karsts tropicaux particulièrement évolués est caractéristique d'une intense dissolution karstique sous une épaisse couche de sédiments meubles (Fenelon, 1951 et 1974). Dans ce type de modelé (karst à hums révélés), qui s'est développé dans un contexte de climat chaud durant le Cénozoïque, les «pechs» correspondent à des buttes de type mogote et les plaines à des paléo-poljés (Salomon, Astruc, 1992). Son aspect actuel a été parachevé durant le climat périglaciaire du 
Quaternaire. Selon S. Konik (1999) : «La localisation des "plaines" et des "pechs" dépend d'anisotropies lithologiques des strates calcaires dont ces derniers constituent les débris. La morphologie très plate des plaines s'explique par le fait que le plancher de la dissolution karstique a atteint le niveau des marnes du Coniacien inférieur. »

Le massif du Pech de l'Azé correspond à la partie haute d'une ride anticlinale (Turq et al., 1999) qui peut être observée le long de la vallée du ruisseau de Fargue et de l'ancienne voie de chemin de fer Sarlat-Gourdon, sur plus d'un kilomètre et demi de long. Aux deux extrémités, elle se termine par des paléo-karsts dans lesquels l'érosion a dégagé des reliefs ruiniformes (lames ou pics rocheux de plusieurs mètres de haut). En son centre, affleurent les marnes de la base du Coniacien inférieur et les bancs de silex qui les surmontent. La présence de cette structure peut, au moins en partie, expliquer la concentration particulièrement importante de sites préhistoriques dans ce secteur. La fracturation des calcaires qu'elle a entraînée a facilité la formation de réseaux karstiques qui sont par la suite devenus des structures d'accueil pour les hommes préhistoriques. De plus, elle explique le nombre de sources du secteur et l'affleurement des silex coniaciens.

Le complexe paléolithique du Pech de l'Azé se compose: - des gisements du Pech de l'Azé I et II qui occupent chacun l'une des extrémités d'une grotte allongée, parallèle à la vallée du ruisseau de Farge;

- de la petite grotte du Pech de l'Azé III située 30 mètres à l'ouest du Pech de l'Azé II;

- du Pech de l'Azé IV, abri effondré qui se trouve à 80 mètres à l'est du Pech de l'Azé I (fig. 1).

\section{HISTORIQUE DES RECHERCHES}

La grotte du Pech de l'Azé I fut découverte dès 1816 par F. V. de Jouannet (Cheynier, 1936). C'est très probablement le premier gisement en grotte qui ait été fouillé dans un but archéologique (Bordes, 1954, p. 428). En 1828, l'abbé Audierne visite ce site et nous apprend qu'une partie du remplissage de la grotte a été détruit quelques années auparavant (Audierne, 1863; Anonyme, 1908). En 1864, E. Lartet et H. Christy le décrivent sous le nom du «Pey de l'Azé». Durant la fin du XIX ${ }^{\mathrm{e}}$ s., plusieurs fouilleurs s'y succèdent et détruisent une partie importante du remplissage.

En 1909, D. Peyrony découvre, à l'entrée, près de la paroi, le crâne d'un jeune enfant néandertalien (Capitan, Peyrony, 1909; Patte, 1957 ; Ferembach et al., 1970 ; Maureille, Soressi, 2000). En 1929 et 1930, R. Vaufrey fouille, à l'avant de la grotte, Pech de l'Azé Ib, plusieurs niveaux qu'il attribue au Moustérien de tradition acheuléenne (MTA) (Vaufrey, 1933).

À partir de 1948, et pour plus d'une trentaine d'années, le complexe paléolithique du Pech de l'Azé est le domaine de F. Bordes. De 1948 à 1951, il entreprend des fouilles toujours à l'avant de la grotte du Pech de l'Azé I (Bordes, 1954, 1955, 1972 et 1978; Bourgon, 1957). L'analyse sédimentologique des dépôts permet de les attribuer au Würm II (Laville et al., 1980). Des fouilles récentes entreprises par M. Soressi (Maureille, Soressi, 2000; McPherron et al., 2001 ; Soressi et al., 2002) et de nouvelles dates ont confirmé que la séquence appartenait à la fin du Paléolithique moyen (Soressi et al., 2008).

En 1948, F. Bordes découvre le gisement du Pech de l'Azé II (Bordes, 1954), grotte déjà visitée par Jouannet (Cheynier, 1936) et il y conduit des fouilles de 1949 à 1951, puis de 1967 à 1969. Des niveaux d'Acheuléen méridional et plusieurs niveaux de Moustérien typique y sont identifiés. La géologie a fait l'objet de deux études (Goldberg, 1979; Texier, 2006). Les datations radiométriques publiées - quatre par les séries de l'uranium pour les sites du Pech de l'Azé I et II (Schwarcz, Blackwell, 1983) et vingt-neuf par résonance paramagnétique électronique (RPE) à partir de dents (Grün et al., 1991) - indiquent que les niveaux archéologiques du Pech de l'Azé II se situent entre les stades isotopiques 6 pour les niveaux 9 à 5 , et les stades isotopiques 5, 4 et 3 pour les niveaux 4 à 2 (Bordes, Bourgon, 1950 et 1951; Laville et al., 1980; Grün et al., 1999).

En 1950, F. Bordes découvre le Pech de l’Azé III, gisement situé à une trentaine de mètres du précédent, toujours au bord de la tranchée de chemin de fer en direction de Sarlat. Cette petite grotte contenait une séquence similaire à celles des niveaux inférieurs du Pech de l'Azé II (Bordes, Bourgon, 1951), à savoir de l'Acheuléen méridional, mais n'a livré que 169 objets lithiques.

Le Pech de l'Azé IV est, quant à lui, découvert en 1952 (Bordes, 1954). De 1953 à 1956, F. Bordes confie la fouille du gisement à B. Mortureux, archéologue amateur sarladais. Ce dernier creuse une longue tranchée perpendiculaire à la paroi, d'un mètre de large et de neuf mètres de long ${ }^{1}$. La présence de gros blocs le force à interrompre ses travaux. F. Bordes reprend les fouilles en 1970. La première année, il élargit la tranchée de son prédécesseur d'un mètre en direction de l'ouest, au nord de la falaise et fait, à l'est,

1. Sur la figure 2, les trois premiers mètres carrés situés en avant du site et correspondant à des zones remaniées n'ont pas été représentés. 


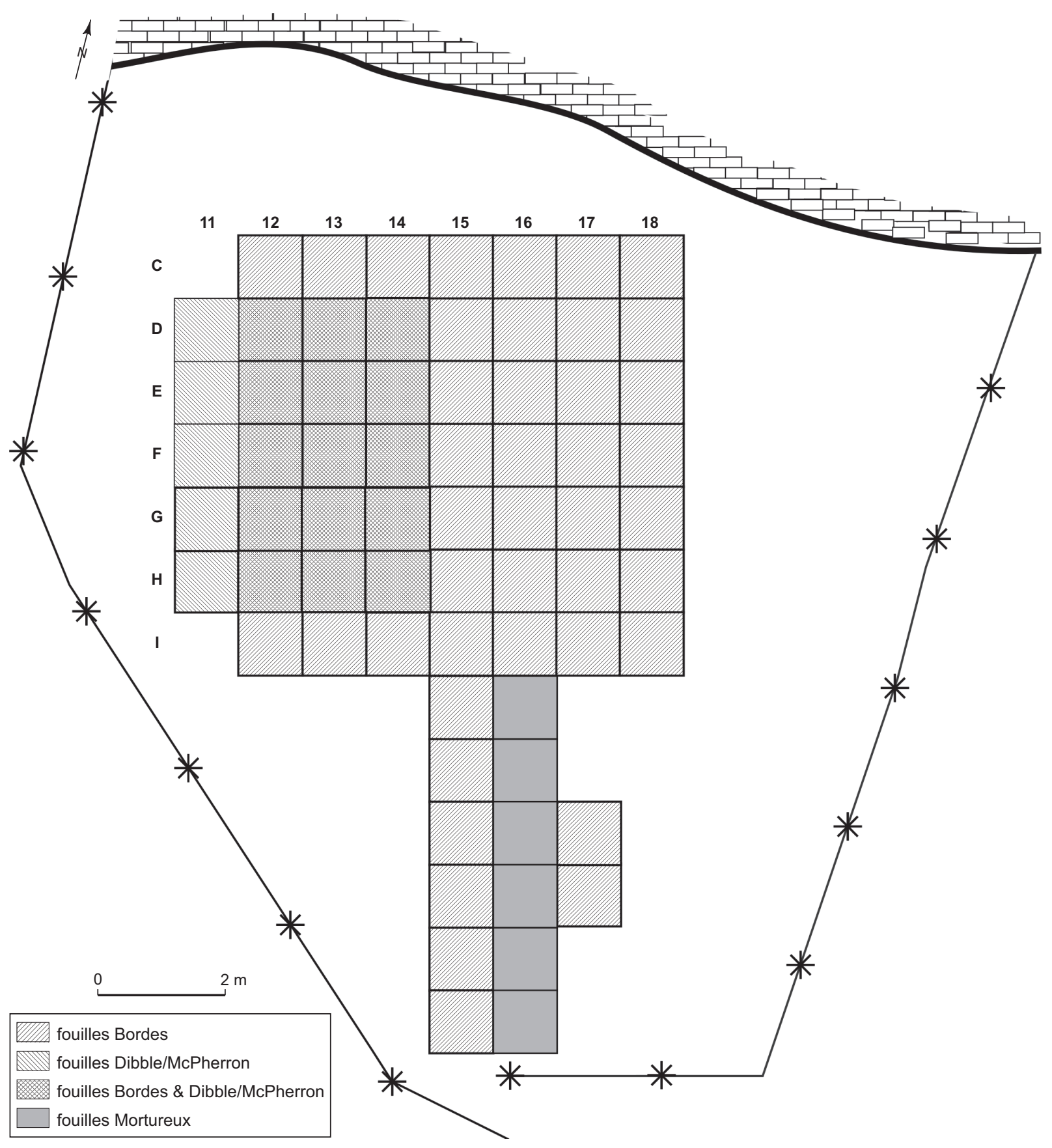

Fig. 2 - Cartographie des carrés d'intervention des différents fouilleurs dans le Pech de l'Azé IV (DAO: H. Dibble, Université de Pennsylvanie).

une encoche d'un mètre de large et deux mètres de long (fig. 2). Les années suivantes, il ouvre une zone de plusieurs mètres carrés près de la falaise. Seul un certain nombre de carrés du côté ouest (C12-I13 et G14-H14) n'ont pas été fouillés jusqu'au sol rocheux. Ils forment ainsi une série de marches descendantes vers la base du gisement. Vers la falaise, le substrat rocheux se situe à $7 \mathrm{~m}$ sous le niveau zéro de la fouille et à $4,5 \mathrm{~m}$ sous la surface originelle. En sept années (de 1970 à 1977), ce sont $52 \mathrm{~m}^{2}$ carrés qui ont été ouverts et un peu moins de $115 \mathrm{~m}^{3}$ de sédiments qui ont été 
explorés. Au vu de l'investissement en temps passé sur la fouille et du nombre d'objets recueillis, le Pech de l'Azé IV représente, après Combe-Grenal à Domme (Dordogne), le plus important gisement que F. Bordes ait fouillé. En raison de sa mort, survenue en 1981, il n'a pas pu mener à terme l'étude de ce site et ne nous a laissé, en tout et pour tout qu'une note (Bordes, 1975), dans laquelle il décrit sommairement la stratigraphie, le matériel lithique et la faune issus des fouilles de 1970-1973. L'industrie moustérienne est attribuée au Moustérien typique, au MTA et un type particulier qualifié d'Asinipodien (Bordes, 1975 et 1981), caractérisé par des nucléus et des éclats Levallois de taille très réduite et par un pourcentage relativement important d'éclats Kombewa.

En 1996, Harold L. Dibble et Shannon P. McPherron ont lancé un nouveau projet de recherche ayant pour but de faire une fouille afin de recueillir de nouvelles données, de mieux comprendre la datation et la formation du gisement et d'être en mesure d'estimer l'intégrité des anciennes collections, de les traiter et les analyser (McPherron, Dibble, 2000).

\section{LES FOUILLES DE F. BORDES}

Des fouilles que F. Bordes dirigea au Pech de l'Azé IV, dans un abri complètement effondré, il nous reste essentiellement la documentation, les collections et une seule note préliminaire (Bordes, 1975).

\section{LA STRATIGRAPHIE DU SITE}

L'unique description, très sommaire, dont nous disposons est un mélange de stratigraphies géologique et archéologique et touche plus particulièrement à la texture et à la composition archéologiques (Dibble, McPherron, 2001; Dibble et al., 2006). Par ailleurs, relevons que le fouilleur lui-même considérait que la stratigraphe du site était difficile: vers l'avant, d'énormes blocs ne permettent pas de suivre certaines couches; le plus souvent, les limites des couches sont indécises, avec passage graduel d'un sédiment à l'autre et variations latérales de faciès (fig. 3).

F. Bordes prend pour exemple la couche I2, composée en avant de menus éboulis concrétionnés et qui, en s'approchant de la falaise, devient plus meuble et passe à des sables rouges plus ou moins durcis.

La stratigraphie que nous allons rappeler ici montre de bas en haut la succession suivante:
- L'ensemble Z est avant tout une couche archéologique qui repose soit sur une mince couche de granules ou directement sur le rocher rougi, par le feu ou par une diagénèse en présence de matière organique, comme à Theopetra en Grèce (Karkanas et al., 1999). L'industrie est du Moustérien typique.

- La couche Y rougeâtre n'existe nettement qu'en avant et semble remplacé vers l'arrière par un éboulis: Moustérien typique.

- La couche X est noirâtre à grisâtre très riche avec au sommet des niveaux à silex cryoturbés: Moustérien typique.

- Une couche d'éboulis correspondant à l'effondrement de l'auvent ou de la paroi.

- Le complexe J3, composé de J3A, J3B, J3C, est renfermé dans un sable assez meuble, à rares éboulis, très riche en silex taillés, débris de faune et traces de foyers. Rougeâtre au sommet, la couche devient grisâtre puis noirâtre à la base. La couche J3 est un Moustérien typique et les couches J3A à J3C correspondent à l'Asinipodien.

- L'ensemble J1-J2: la couche J2 présente dans une matrice sableuse des éboulis arrondis et des silex concassés par cryoturbation. Ce phénomène semble plus important à l'avant du site que près de la falaise où les éboulis sont anguleux et les silex presque intacts. La couche J1 est constituée de sable rouge-brun clair avec gros éboulis épars témoignant d'un nouvel effondrement de la paroi. L'industrie, peu riche, est attribuable au Moustérien typique.

- L'ensemble I1-I2: la couche I2 est caractérisée par des petits éboulis plus nombreux qu'en I1. Ces deux couches, fortement concrétionnées, contiennent deux riches ensembles de Moustérien typique classique.

- L'ensemble H, composé des couches H1 et H2, est sableux avec des éboulis épars. Selon le fouilleur, il s'agit de deux niveaux de Moustérien typique.

- La couche G pose problème. En dehors de l'endroit où existent des poches riches en matériel archéologique, la couche est presque stérile. F. Bordes pense, en se basant sur la typologie, que les objets conservés proviennent de la couche F4.

- L'ensemble F est un éboulis menu vers l'arrière et un éboulis de taille moyenne en avant. Il est divisé de manière semi-artificielle en F1, F2, F3 et F4. L'industrie, en particulier en F4, est la plus riche du gisement. Elle est attribuable à un MTA avec une évolution interne du MTA A (niveau F4) au MTA B (niveaux F2 et F1).

- L'ensemble E et D est composé de sables rougeâtres avec éboulis: présence de Moustérien. 


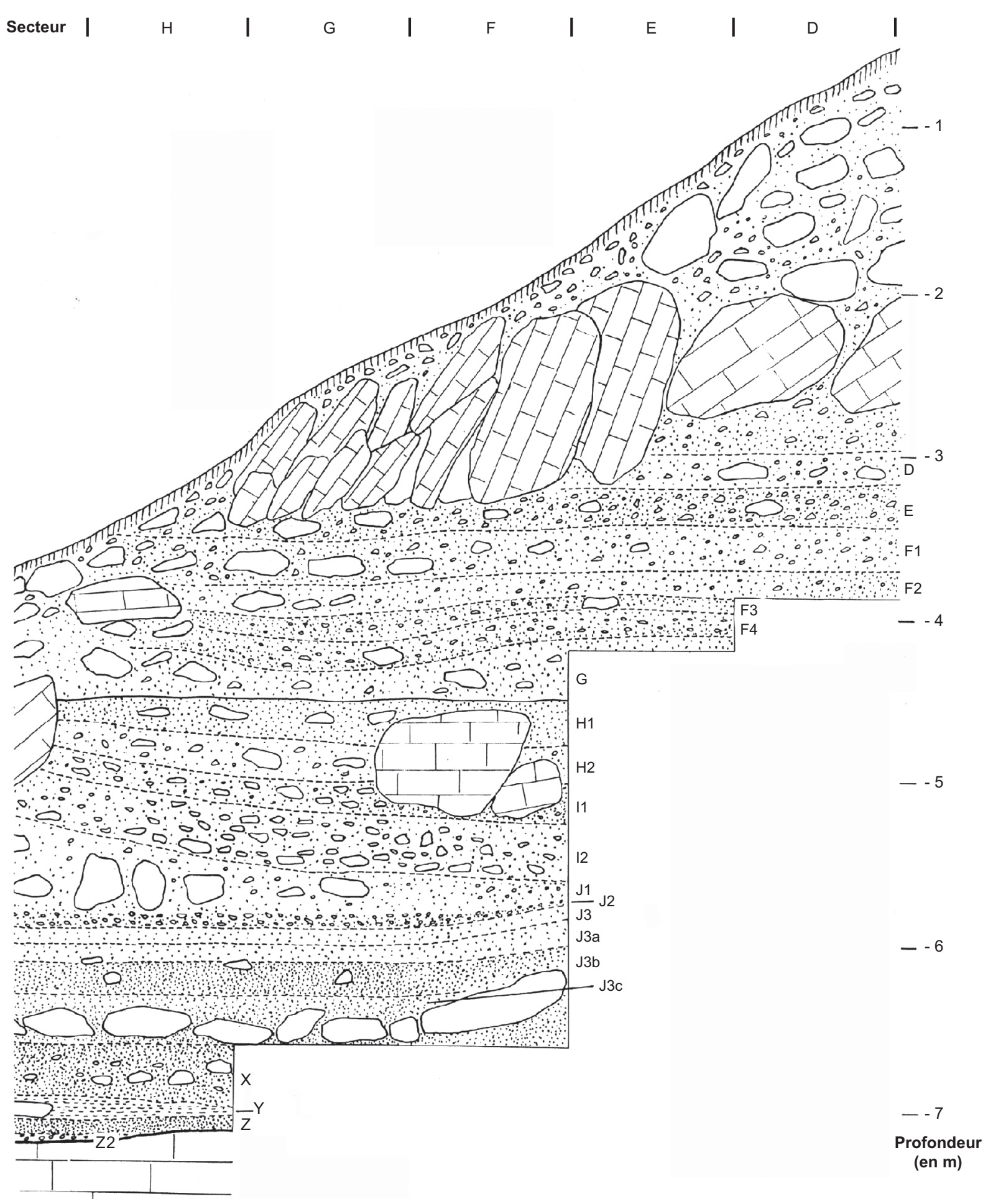

Fig. 3 - Stratigraphie du Pech de l’Azé IV relevée par F. Bordes (DAO: H. Dibble, Université de Pennsylvanie). 
- Les couches B et C de sables bruns sont remplacées, dans une partie de la fouille, par de gros blocs, ultime effondrement de la voûte ou de la paroi: quelques traces de Moustérien.

- La couche A2, composée de sable brun et d'éboulis, n’a livré qu'un seul éclat.

- La couche A1, couche végétalisée, contient quelques silex et des fragments de poterie datant du Moyen Âge, voire même de l'âge du Fer.

\section{LES DONNÉES DE LA FAUNE}

Les restes fauniques ne sont connus qu'à travers un bref résumé des espèces présentes dans chacun des niveaux (Bordes, 1975) et par le travail réalisé par G. Laquay sur les niveaux inférieurs (Laquay, 1981). Dans les niveaux Z à J2, le Cerf (Cervus elaphus) est dominant. Il est toujours abondamment représenté dans les niveaux supérieurs mais le Renne apparaît en J3A et domine de I2 à F4. Les Bovidés sont fréquents en J1, I2 et en F. Ils dominent en F1. Le Chevreuil est fréquent en $\mathrm{X}$, atteint un maximum en J3 et diminue dans les niveaux supérieurs. Le Cheval est aussi relativement fréquent en $\mathrm{Z}$ et en Y. Il diminue ensuite.

\section{PROPOSITION DE CORRÉLATIONS DU PECH DE L'AZÉ I AVEC LES AUTRES SITES DU COMPLEXE}

F. Bordes, en s'appuyant sur la faune et les industries lithiques mais surtout sur les travaux de Laville (1973a et b), proposa quelques corrélations (tabl. I). Il fit un parallèle entre les niveaux Z-G du Pech de l'Azé IV avec les niveaux supérieurs du Pech de l'Azé II. Les niveaux X, Y et Z correspondraient aux niveaux 4A-4E du Pech de l'Azé II, ce qui implique un âge d'environ 80000 ans; les effondrements du plafond du Pech de l'Azé IV entre les niveaux X et J3 correspondraient à ceux du niveau 3 au Pech de l'Azé II, situé aux environs de 65000 ans. Enfin, les niveaux J à G ont été rapprochés des sous-niveaux du niveau 2 du Pech de l'Azé II, pour lesquels les datations par résonance de spin électronique (RSE) ont donné un âge compris entre 54000 ans et 59000 ans (Grün et al., 1991); et les niveaux F ont été corrélés aux niveaux MTA du Pech de l'Azé I, datés entre 41000 ans et 51000 ans (Soressi et al., 2008). Ces parallèles ont généralement été fortement critiqués (Mellars, 1988; Texier, 2000; Bertran, Texier, 1995), ce qui nous conforte dans notre volonté d'obtenir de nouvelles dates radiométriques de la séquence.

\section{LES COLLECTIONS F. BORDES}

Lorsqu'en 1996, nous avons commencé à travailler sur le site, quel était l'état des collections (environ 6000 os et près de 60000 objets lithiques)?

Une moitié du matériel avait été lavée, étiquetée par carré et numéro d'identification, puis organisée par F. Bordes par niveaux et classes typologiques. L'autre moitié n'était ni lavée, ni marquée et les objets étaient sans provenance précise. Enfin, les petits éléments, objets lithiques et faune mélangés, étaient stockés dans des sacs en plastique marqués par carré, ou partie de carré, et par profondeur.

\begin{tabular}{|c|c|c|c|c|c|c|}
\hline \multirow{2}{*}{ Phase climatique } & \multicolumn{2}{|c|}{ Pech I } & \multicolumn{2}{|c|}{ Pech II } & \multicolumn{2}{|c|}{ Pech IV } \\
\hline & couche & industrie & couche & industrie & couche & industrie \\
\hline Würm II, Périgord III-VIII & $\begin{array}{l}7 \\
6\end{array}$ & MTA-A & & & $\begin{array}{l}\text { F1 } \\
\text { F2 }\end{array}$ & MTA-B \\
\hline Würm II, Périgord II & 5 & MTA-A/B & & & F3 & MTA-A/B \\
\hline Würm II, Périgord I & $\begin{array}{l}4 \\
3\end{array}$ & MTA-A & & & F4 & MTA-A \\
\hline Würm I, Périgord IX & & & $2 \mathrm{~A}-2 \mathrm{C}$ & moustérien & G & moustérien \\
\hline Würm I, Périgord VIII & & & $2 \mathrm{D}$ & moustérien & $\mathrm{H} 1-\mathrm{H} 2$ & typical \\
\hline Würm I, Périgord VII & & & $2 \mathrm{E}-2 \mathrm{~F}$ & typical & $|1-| 2$ & typical \\
\hline Würm I, Périgord V-VI & & & \begin{tabular}{|c|}
$2 G$ \\
$2 G^{\prime}$ top \\
\end{tabular} & moustérien & J1-J2 & typical \\
\hline Würm I, Périgord IV & & & $\begin{array}{c}2 G^{\prime} \\
\text { base }\end{array}$ & moustérien & $\begin{array}{c}\mathrm{J3} \\
\mathrm{J} 3 \mathrm{a}-\mathrm{C}\end{array}$ & $\begin{array}{c}\text { typical } \\
\text { asinipodien }\end{array}$ \\
\hline Würm I, Périgord III & & & 3 & typical & & \\
\hline Würm I, Périgord II & & & $\begin{array}{l}\mathrm{AA} \\
4 \mathrm{~B} \\
4 \mathrm{C}\end{array}$ & $\begin{array}{c}\text { typical } \\
\text { denticulés } \\
\text { typical }\end{array}$ & $x$ & typical \\
\hline Würm I, Périgord I & & & $\begin{array}{l}\mathrm{DD} \\
4 \mathrm{E}\end{array}$ & typical & $\begin{array}{l}\mathrm{Y} \\
\mathrm{Z}\end{array}$ & typical \\
\hline
\end{tabular}

Tabl. I - Corrélations chronologiques proposées par F. Bordes pour l'ensemble des sites du Pech en prenant en compte l'industrie, la faune et la géologie (d'après F. Bordes, 1975). 
Ces sacs et leur contenu ont été inventoriés, les objets lithiques de taille supérieure à 2,5 cm ont été récupérés et de nouveaux numéros d'identification leur ont été attribués.

Les carnets de fouille renfermant toutes les coordonnées, les dessins et les notes de fouilles furent maintenus en bon état par D. de Sonneville-Bordes et conservés au musée d'Aquitaine à Bordeaux. Au total, il s'agit d'environ 2500 pages de notes prises sur le terrain (plans divers et schémas stratigraphiques). Toutes ces données ont été informatisées. Nous avons enregistré, pour chaque artefact, le carré, le numéro d'identification, les coordonnées X (par rapport au bord ouest du carré), Y (par rapport au bord sud du carré), Z (par rapport au système de référence du site), un code indiquant la nature de l'artefact (outil retouché, éclat, nucléus, dent, etc.), une description du sédiment, le niveau, le nom du fouilleur et la date. Nous avons ensuite converti toutes les coordonnées dans un nouveau carroyage englobant l'ensemble du site, prenant comme référence le point zéro du site situé sur la falaise surplombant celui-ci.

\section{PRÉPARATION DES COLLECTIONS D'INDUSTRIE LITHIQUE}

En inventoriant et en analysant les collections, deux problèmes importants ont été rencontrés. Le premier est le nombre relativement important de doublons (objets présentant le même numéro) qui n'est apparu qu'après informatisation de l'ensemble de la collection. La production de doublons peut résulter de plusieurs facteurs: deux objets peuvent être ensachés ensemble sur le terrain (souvent lorsque le fouilleur est convaincu qu'il s'agit des morceaux d'un même objet). Lors des opérations de lavage et de marquage, des erreurs de lecture ou de reports du numéro d'identification peuvent se produire ${ }^{2}$, le dernier numéro d'identification utilisé étant oublié, les numéros d'identification déjà utilisés sont ainsi de nouveau employés. S'il est facile de créer des numéros en double, il peut être très difficile de corriger ces erreurs, surtout si cela n'est pas fait tout de suite. Ici, cela a été impossible. Nous avons donc décidé d'éliminer tous les doublons, soit près de $10 \%$ du matériel lithique.

Le second problème a été d'attribuer les objets à l'un des niveaux archéologiques. Nous avons utilisé pour cela

2. Pour remédier à ce problème au cours de nos propres projets sur le terrain, nous avons mis en place un système dans lequel une personne marque l'artefact et une deuxième lit le numéro et vérifie qu'il correspond bien à celui inscrit sur l'étiquette. De cette manière, la quasitotalité des erreurs de marquage est éliminée. trois sources d'information: les attributions réalisées par F. Bordes avant sa mort (les objets ont été stockés ensemble, le niveau étant indiqué sur leur unité de stockage), les notes prises sur le chantier par chaque fouilleur et la position des artefacts dans un système tridimensionnel. En utilisant l'ensemble de ces sources, il est possible d'attribuer un contexte stratigraphique à la quasi-totalité des artefacts.

Ce travail a par ailleurs mis en évidence un autre problème. Comme nous l'avons déjà mentionné, environ la moitié de la collection a été organisée par F. Bordes et stockée dans des tiroirs, chaque tiroir portant une étiquette indiquant le niveau (les objets eux-mêmes n'ayant pas été marqués selon le niveau). En regardant la position tridimensionnelle de ces objets (fig. 4), il est apparu clairement qu'au moins deux tiroirs avaient été mal identifiés (étant donné que ces niveaux étaient plus ou moins horizontaux). Sans cette vérification de la provenance exacte des objets selon les coordonnées $\mathrm{X}, \mathrm{Y}$ et $\mathrm{Z}$, cette erreur serait passée inaperçue et aurait été une source de confusions dans la collection (Dibble et al., 2005).

\section{INTÉGRITÉ DE LA STRATIGRAPHIE}

Le point le plus important à retenir est que la séquence stratigraphique telle qu'elle a été décrite par F. Bordes, et rappelée ci-dessus, s'étend sur tout le site (fig. 5). En d'autres termes, il est possible de suivre la plupart des niveaux de la coupe ouest à la coupe est en passant par la coupe nord. Les principales exceptions sont les niveaux supérieurs F qui ne sont pas représentés dans la partie est de la coupe nord (à cause de la présence du socle rocheux à cet endroit), alors qu'ils sont représentés de façon limitée dans la coupe est. De la même manière, les niveaux $\mathrm{X}, \mathrm{Y}$ et $\mathrm{Z}$ ne sont pas représentés dans la coupe nord à cause d'une remontée subite du substratum calcaire. Ainsi, contrairement aux rumeurs qui circulent depuis la fermeture du site dans les années 1980, il ne nous semble pas que l'intégrité stratigraphique du site puisse être remise en cause.

\section{LES FOUILLES DE 2000-2003}

De nouvelles fouilles ont été entreprises au Pech de l'Azé IV à partir de 2000. Durant les quatre campagnes de terrain, la plus grande partie de notre travail s'est concentrée sur la portion ouest du site, dans la continuité des fouilles de F. Bordes arrêtées en 1977. Cela a inclus la fouille d'une «banquette» laissée par le précédent fouilleur, principalement dans les carrés D12 à F14, et la fouille de la séquence 


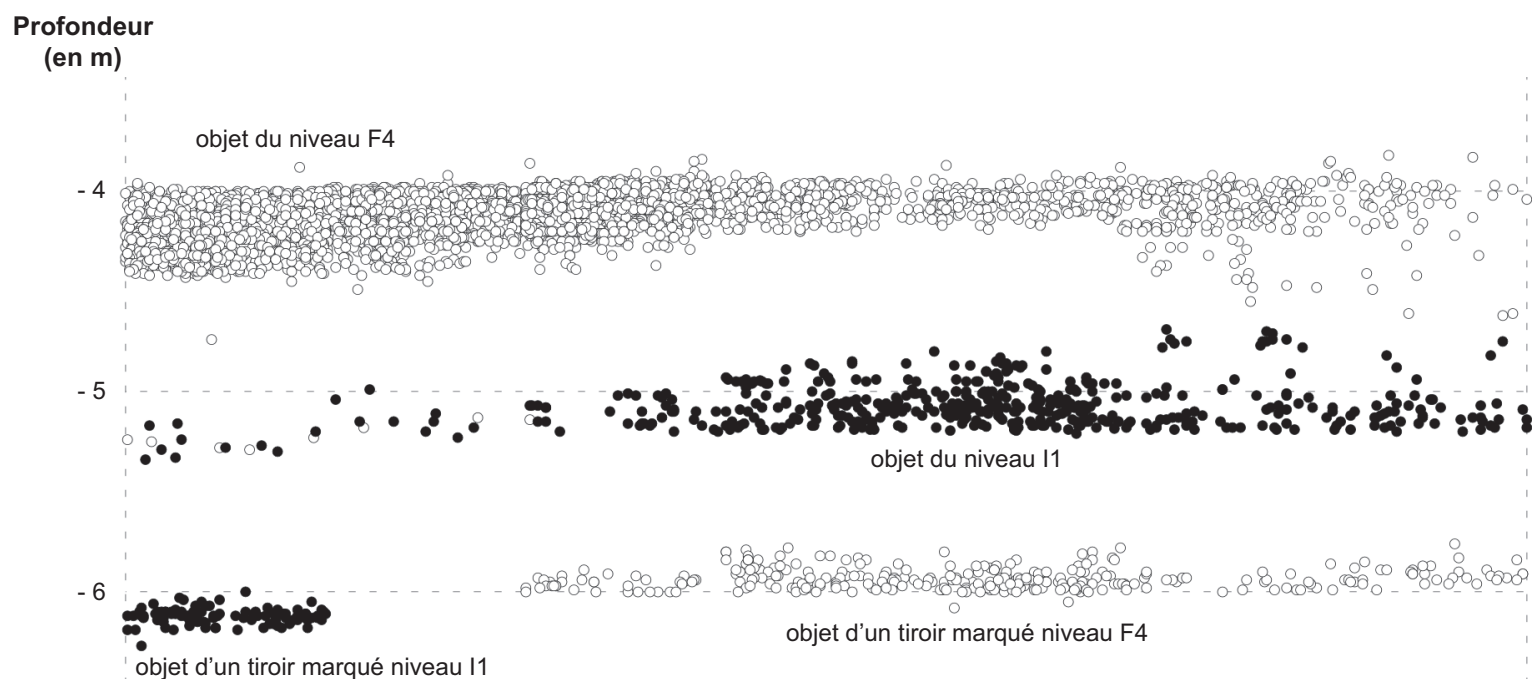

Fig. 4 - Illustration d'un problème de conservation des collections F. Bordes: erreur d'étiquetage de tiroir. Mise en évidence lorsque les artefacts ont été placés dans les diagrammes de projection à partir des données des carnets de fouilles (DAO: H. Dibble, Université de Pennsylvanie).

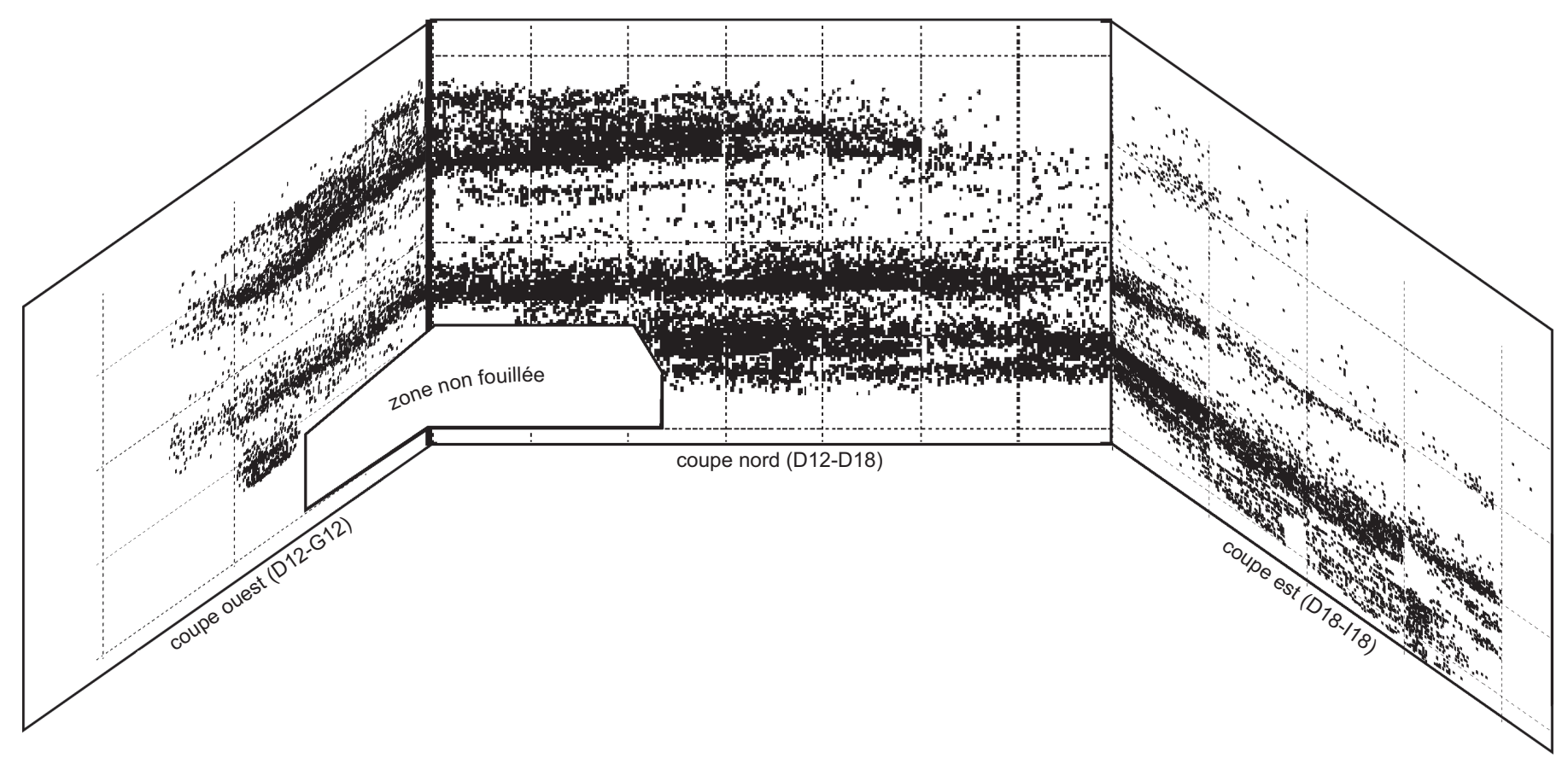

Fig. 5 - Diagrammes de projection des objets provenant de la proximité des trois coupes laissées par F. Bordes (DAO: H. Dibble, Université de Pennsylvanie). 
complète le long de la coupe ouest, définie par les carrés D11 à H11 (fig. 2). Nous avons également laissé une petite banquette composée des sédiments du niveau le plus inférieur (niveau 8 dans notre système stratigraphique) et qui contenait de nombreux foyers superposés (voir infra, p. 17-19). Nous avons pensé que leur potentiel serait mieux compris par les méthodes pouvant être développées dans l'avenir.

\section{MÉTHODOLOGIE}

La méthode de fouille utilisée est celle que nous employons depuis vingt ans sur les sites du Paléolithique ancien et moyen français de la Quina à Gardes-le-Pontaroux (Charente), Combe-Capelle bas à Saint-Avit-Sénieur (Dordogne), l'Epinette à Cagny (Somme), Fontéchevade à Montbron (Charente), Roc de Marsal à Campagne (Dordogne), et Chez-Pinaud à Jonzac (Charente-Maritime), parmi d'autres sites (Dibble, Lenoir, 1995; Dibble et al., 1997; McPherron, Dibble, 2002).

\section{TEGHNIQUES DE FOUILLE}

Les objets intéressants ainsi que les échantillons et les prélèvements sont tous enregistrés en trois dimensions mais traités de différentes façons en fonction de leur nature et leur type:

- Objet numéroté: il lui est assigné un numéro d'identification par carré et ses coordonnées;

- échantillon de tamis: la totalité du sédiment ainsi que les petits objets de moins de $25 \mathrm{~mm}$ récoltés dans les seaux pendant la fouille. Chaque seau possède son propre numéro d'identification par carré et il a pour localisation le centre de la zone en cours de fouille (généralement un quart de mètre carré) ;

- objets naturels (blocs, éboulis...) : ils sont enregistrés par carré avec un identifiant non numérique mais ils ne sont généralement pas conservés; plusieurs visées permettent d'obtenir un dessin schématique de l'objet;

- échantillons quotidiens de sédiment: ils sont localisés et possèdent un numéro unique d'identification par carré;

- échantillons pour datation par thermoluminescence, résonance de spin électronique (RSE) et ${ }^{14} \mathrm{C}$ : ils sont photographiés sur place, un numéro unique d'identification leur est attribué par carré;

- autres échantillons (micromorphologie, géophysique...) : ils possèdent un numéro unique d'identification par carré; - les points topographiques sont pris au sommet de chaque décapage.

\section{GRITÈRES DE DÉFINITION DES OBJETS NUMÉROTÉS}

Les objets appartenant aux catégories suivantes ont été enregistrés et numérotés :

- Les objets lithiques travaillés mesurant $25 \mathrm{~mm}$ ou plus, les nodules de silex au-delà de $10 \mathrm{~cm}$ dans leur dimension maximale et les outils retouchés quelle que soit leur dimension.

- Les galets de quartz de plus de $25 \mathrm{~mm}$.

- Les minéraux étrangers au site et aux sédiments qu'il renferme (ocre, manganèse...), peu importe leur taille.

- Les os entiers ou fragmentés supérieurs à $25 \mathrm{~mm}$ dans leur dimension maximale; toutes les dents et les petits os identifiables (sésamoïdes, carpes...), peu importe leur dimension.

Ajoutons que les objets retrouvés au cours du tamisage à l'eau ont un numéro d'identification associé à celui du seau de sédiments dans lequel ils se trouvaient.

\section{MÉTHODE D'ENREGISTREMENT DES OBJETS}

L'ensemble des coordonnées des objets est enregistré à l'aide d'une station totale (un théodolite électronique intégrant un télémètre laser). Cet outil est extrêmement précis (un millimètre avec une exactitude d'environ $+/-2 \mathrm{~mm}$ ) et rapide dès lors qu'il est couplé à un petit ordinateur portable (Dibble, 1987; McPherron, Dibble, 1987 et 2002; Dibble, McPherron, 1988 et 1992; Dibble et al., 1995).

La plupart des objets sont enregistrés avec une seule visée prise au centre et directement en dessous de celui-ci. De plus, tout objet déplacé lors de la fouille, quelle que soit sa taille, est enregistré avec une seule visée, correspondant au centre du carré. Les objets allongés sont enregistrés avec deux visées prises aux extrémités en plaçant la pointe du prisme sur la surface fouillée juste à côté de l'objet (McPherron, 2005).

Dès lors qu'un objet est enregistré dans ses trois dimensions et possède un numéro d'identification, d'autres informations sont prises en compte et entrées directement dans l'ordinateur relié à la station: le niveau archéologique dans lequel l'objet a été mis au jour; un code indiquant la nature de l'objet identifié; les noms du fouilleur et la date de la fouille. Ce code a été vérifié par nos soins au cours d'un inventaire général du matériel au laboratoire. L'utilisation de ce système informatisé d'enregistrement des données de terrain, ainsi que l'enregistrement de plusieurs points pour les objets de grande taille, éliminent la néces- 
sité de dessiner à la main tous les objets archéologiques au fur et à mesure de leur découverte.

\section{ÉTIQUETTES ET CODES-BARRES}

Une nouvelle méthodologie est employée pour la fouille du Pech de l'Azé IV: l'utilisation d'étiquettes avec codesbarres pour les objets numérotés (Dibble, McPherron, 2007). Cette technique permet d'une part de diminuer de façon importante le risque de doublons et d'autre part les erreurs d'entrée des données dans l'ordinateur. Enfin la vitesse d'enregistrement est sérieusement accrue. Dès qu'un objet est découvert et enregistré, il est mis en sachet individuel avec une étiquette séquentielle. Ce système permet une double vérification du numéro d'identification de l'étiquette créé par l'ordinateur au moment de son enregistrement par la station totale.

$\mathrm{Au}$ laboratoire, dès lors que l'objet est lavé et marqué, une nouvelle étiquette est imprimée. La première est scannée et le logiciel recherche dans la base de données les informations associées à cet objet. Une nouvelle étiquette prenant en compte la nature de l'objet (osseuse, lithique, manuport...) est éditée et placée à l'extérieur d'un nouveau sachet. L'utilisation de ces étiquettes n'élimine en aucun cas la nécessité de marquer l'objet avec son numéro d'identification et son carré. Elles sont davantage utilisées comme un moyen de suivre le niveau d'analyse effectué sur chaque objet. Ce système s'est révélé non seulement très efficace au niveau du traitement des données, mais aussi au niveau de la conservation du matériel en permettant de réduire les éventuels dégâts causés par le stockage.

\section{ÉCHANTILLONS DU TAMISAGE À L'EAU}

Les seaux servent à associer les objets de petite taille (moins de $25 \mathrm{~mm}$ ), ceux déplacés durant la fouille avant l'enregistrement, et le matériel archéologique récupéré au cours du tamisage à l'eau, avec le carré d'origine. Tous possèdent un numéro d'identification qui permet de replacer globalement et de façon tridimensionnelle les objets qu'ils renferment.

Les seaux contiennent environ sept litres de sédiments qui proviennent normalement d'une même zone de $50 \mathrm{~cm}$ de diamètre et d'un même niveau. Ils sont ramenés au laboratoire pour être passés à l'eau à travers deux tamis, l'un à maille de $6 \mathrm{~mm}$ et l'autre de $2 \mathrm{~mm}$. Après séchage, les refus de tamis sont triés et le matériel, os et lithique, mis en sachets séparément. Les objets trouvés au cours de cette opération, qui auraient dû être enregistrés, sont isolés. Un numéro d'identification leur est attribué, leurs coordonnées sont celles du seau. Ils sont alors traités comme tous les autres objets archéologiques.

\section{PREMIERS RÉSULTATS}

\section{LES PROCESSUS DE FORMATION DU SITE (STRATIGRAPHIE ET MICROMORPHOLOGIE DES SOLS)}

L'un des objectifs majeurs du projet de reprise des fouilles au Pech de l'Azé IV était notamment d'analyser les processus de formation du site, leurs implications et leurs effets sur les assemblages archéologiques. L'approche géo-archéologique dans son ensemble vise à reconstruire les caractéristiques du site au cours de son occupation et déterminer les processus naturels qui ont pu le modifier au cours du temps, à mettre en corrélation les sections est et ouest du site et à identifier l'origine et l'environnement des dépôts des sédiments rougeâtres, et les relations entre Pech de l'Azé I, II et IV (Goldberg, 1979; Goldberg, Macphail, 2003; Ortoli, 2004). L'analyse micromorphologique du sol complétée par des analyses géochimiques, comme la spectroscopie infrarouge à transformée de Fourier (FTIR) et la microsonde électronique (non abordée ici), permettent de préciser l'état de conservation du site: les effets du gel et du dégel, la dissolution des carbonates et les activités humaines, telles que la vidange des foyers et le piétinement.

\section{MÉTHOdeS}

Les méthodes macroscopiques de terrain incluent les comparaisons entre le Pech de l'Azé IV et la lithologie locale au moyen de cartes, l'observation sur le terrain, et les travaux précédents effectués sur la stratigraphie du Pech de l'Azé II par H. Laville (1973) et P. Goldberg (1979). Les caractéristiques macroscopiques des sédiments ont été utilisées sur le terrain pour reconnaître les divisions stratigraphiques originelles de F. Bordes et vérifier leur pertinence.

La principale technique d'analyse employée est la micromorphologie du sol. Elle étudie la structure des sédiments en lames minces, en utilisant un microscope pétrographique afin de reconstituer la formation des unités géologiques, archéologiques ou pédologiques. En 2001, 2002 et 2003, environ deux cents blocs de sédiments intacts ont été prélevés selon la procédure décrite par P. Goldberg 
et R. Macphail (2003). Des échantillons de sédiments en vrac ont également été recueillis pour d'éventuelles analyses FTIR, diffraction des rayons X sur poudre (XRD). Ces prélèvements ont été transportés à l'université de Boston, séchés pendant une semaine dans un four à $60^{\circ} \mathrm{C}$, puis imprégnés dans un mélange de résine de polyester non accélérée de styrène ( 7 pour 3 ) et dans un catalyseur MEKP qui a permis leur durcissement après une période de quatre à cinq jours. Après une seconde cuisson d'une journée dans un four à $60^{\circ} \mathrm{C}$, les blocs ont été tranchés à l'aide d'une scie diamantée, des zones sélectionnées et préparées en lames minces de $50 \mathrm{~mm}$ x $75 \mathrm{~mm}$ en vue d'analyses au Spectrum Petrographics.

$\mathrm{Au}$ total, ce sont environ 129 lames minces qui ont été étudiées avec un microscope pétrographique à grossissement variable, en lumière naturelle $(\mathrm{LN})$ et en lumière polarisée (LP), ainsi qu'en lumière incidente oblique (OIL), utilisée pour distinguer les matières organiques (charbons brûlés ou humifiés). Une visionneuse de microfiche a également été employée pour analyser la forme des vides et déterminer la microstructure des sédiments. L'autofluorescence a révélé la présence de phosphates primaires (os, coprolithes) et secondaires (croûtes apatitiques). Les analyses FTIR des échantillons de sédiments en vrac ou en poudre ont été réalisées à l'université d'Arizona à l'aide d'un spectromètre Thermo Nicolet avec un cristal ATR en diamant (réflectance totale atténuée).

\section{OBSERVATIONS SUR LA NATURE DU SITE}

F. Bordes décrit initialement Pech de l'Azé IV comme l'effondrement d'un abri sous roche qui était géomorphologiquement sans rapport avec les grottes communicantes du Pech de l'Azé I et II.

La nouvelle fouille a révélé que Pech de l'Azé IV est, en fait, une grotte effondrée, identique à celles du Pech de l'Azé I et II, ce qui permet de penser que toutes ces cavités appartiendraient au même système karstique et ont pu l'être, à un moment de leur histoire. Cette hypothèse s'appuie sur une série de données:

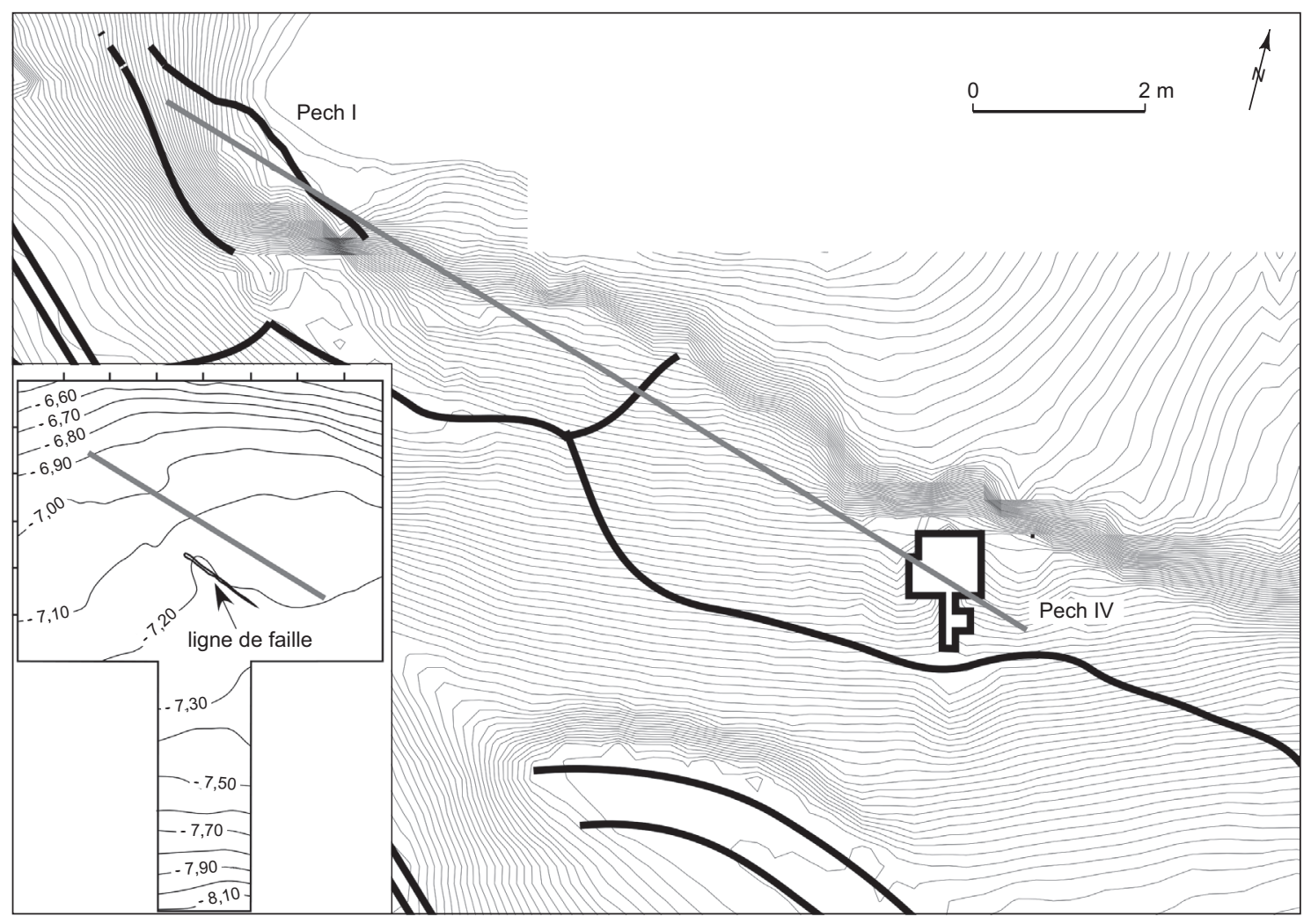

Fig. 6 - Relevé topographique du secteur entre le Pech de l'Azé I et IV; en bas à gauche, topographie du sol rocheux atteint lors des fouilles; en gris, l'axe karstique à l'origine de la formation du Pech de l'Azé IV (DAO: H. Dibble, Université de Pennsylvanie). 
- La pente du socle rocheux s'oriente de l'intérieur vers l'entrée (du nord au sud), elle est associée aux dépôts de sédiments qui présentent la même inclinaison (fig. 6). En effet, les sédiments d'un abri sous roche s'accumulent communément au-dessous du surplomb, formant un cône de talus à partir duquel ils sont transportés en aval ;

- les artefacts et les os présentent la même orientation, s'inclinant vers le sud en direction de l'entrée. De plus, les grands axes des artefacts ont tendance à être légèrement orientés de l'est vers l'ouest, ce qui pourrait évoquer un faible débit interne entre Pech I, II et IV (McPherron, 2005); - les vestiges macroscopiques de la grotte originelle, y compris des fragments de stalagmite et de travertin (formés par les lignes de gouttière dans l'espace de l'ancienne grotte), ont été retrouvés dans des sédiments qui ne sont pas recouverts par le toit actuellement (fig. 7).

Après la fouille des niveaux les plus bas, nous avons observé une microfaille du socle rocheux (fig. 8). Elle est parallèle à l'orientation des axes de Pech de l'Azé I et II (fig. 6). Sa présence permet d'envisager, dans le prolongement du site, l'existence d'un possible réseau karstique. N'oublions pas que nous sommes dans un anticlinal (Turq et al., 1999), il n'est donc pas rare d'observer, dans ce type de structure, le développement de karst à partir de failles ou fissures.

Les changements de la morphologie de la grotte supposée ont contribué, au fil du temps, à une sédimentation géogénique du site, comme le montrent les épisodes plus ou moins importants d'effondrement du toit. Certains épisodes et l'accumulation de gros blocs résultant de l'écroulement du plafond ont, sans aucun doute, modifié la structure d'accueil des hommes et l'organisation des dépôts.

\section{RÉVISION DE LA LITHOSTRATIGRAPHIE}

La stratigraphie actuelle prend en compte des critères lithostratigraphiques tels que la couleur, la composition, la texture, la morphologie des grains et la structure interne.

Sur le socle rocheux repose la couche 8 , couche massive à grossière, de couleur brun rougeâtre foncée, composée de sables organiques gras (fig. 9). Elle renferme quelques niveaux rougeâtres centimétriques, et des lentilles apparemment plus riches en cendres et en charbons de bois (fig. 10). Le matériel grossier est principalement d'origine anthropique et contient peu d'éléments de calcaires. La base, localement très poreuse, est indurée par le carbonate, alors que le sommet présente une forte augmentation des composants anthropiques. Cette couche est particulièrement développée dans le sud-ouest du site (carrés G12 à G14, H11 à H14, I12 à I14), l'action du feu a affecté plus de $20 \%$ des artefacts.

La couche 7 se caractérise par la présence de nombreux fragments de silex aux bords endommagés. Plus claire que la couche 8 , elle semble contenir moins de matériel organique et de fraction fine, malgré des proportions similaires des autres composants anthropiques (os brûlés, cendres et silex). Son épaisseur varie entre $5 \mathrm{~cm}$ et $10 \mathrm{~cm}$. Le contact entre 7 et 8 est généralement plat et net (fig. 11), mais peut être localement ondulé. Dans la partie nord du site, la couche 7 repose directement sur le socle rocheux, alors que dans la partie sud, elle est présente par intermittence, comme des taches entre les blocs d'effondrement du toit.

La couche 6 se subdivise en deux parties. À la base, $6 \mathrm{~B}$ correspond à de grandes plaques calcaires issues de l'effondrement du plafond, elles reposent directement sur la couche 7 (fig. 11). Les blocs les plus grands, concentrés dans la partie ouest du site, peuvent avoir une épaisseur d'environ 20 à $30 \mathrm{~cm}$ et atteindre jusqu'à $120 \mathrm{~cm}$ de long. Cette couche renferme également des petits blocs calcaires, des galets et des graviers, ainsi que des composants anthropiques (en particulier sur le profil est).

La partie supérieure, 6A, est formée de fragments de l'effondrement du plafond contenus dans un sable jaunerouge à brun foncé. Cette couche présente des indices de bioturbation ou de cryoturbation (comme l'inclinaison des artefacts).

Entre les couches 6 et 5 se place une phase de lacune stratigraphique.

La couche 5 est également subdivisée en $5 \mathrm{~A}$ et $5 \mathrm{~B}$. Globalement, elle s'incline vers l'ouest et le sud-ouest et présente une matrice limoneuse à sablonneuse rouge jaunâtre. Son contact avec $6 \mathrm{~A}$ est net et irrégulier.

La base 5B, dont l'épaisseur varie entre 15 et $40 \mathrm{~cm}$, contient des clastes et plusieurs galets calcaires exceptionnellement bien arrondis et durs. Initialement interprétés comme résultant de la cryoturbation, ces galets calcaires sont aujourd'hui envisagés comme étant des éléments retravaillés et issus des dépôts phréatiques grossiers de l'intérieur de la grotte. Le même type d'événement a été documenté dans le sud-ouest de la Suisse (Braillard et al., 2004).

La couche 5A contient des blocs angulaires issus de l'effondrement du plafond. Ils ne résultent pas d'un seul événement, mais plutôt d'une série de petits épisodes. Au sud, vers l'entrée de la grotte, leur imbrication indique qu'un gros bloc est tombé en premier lieu de la voûte à l'intérieur. D’autres blocs se sont ensuite détachés du plafond affaibli et 


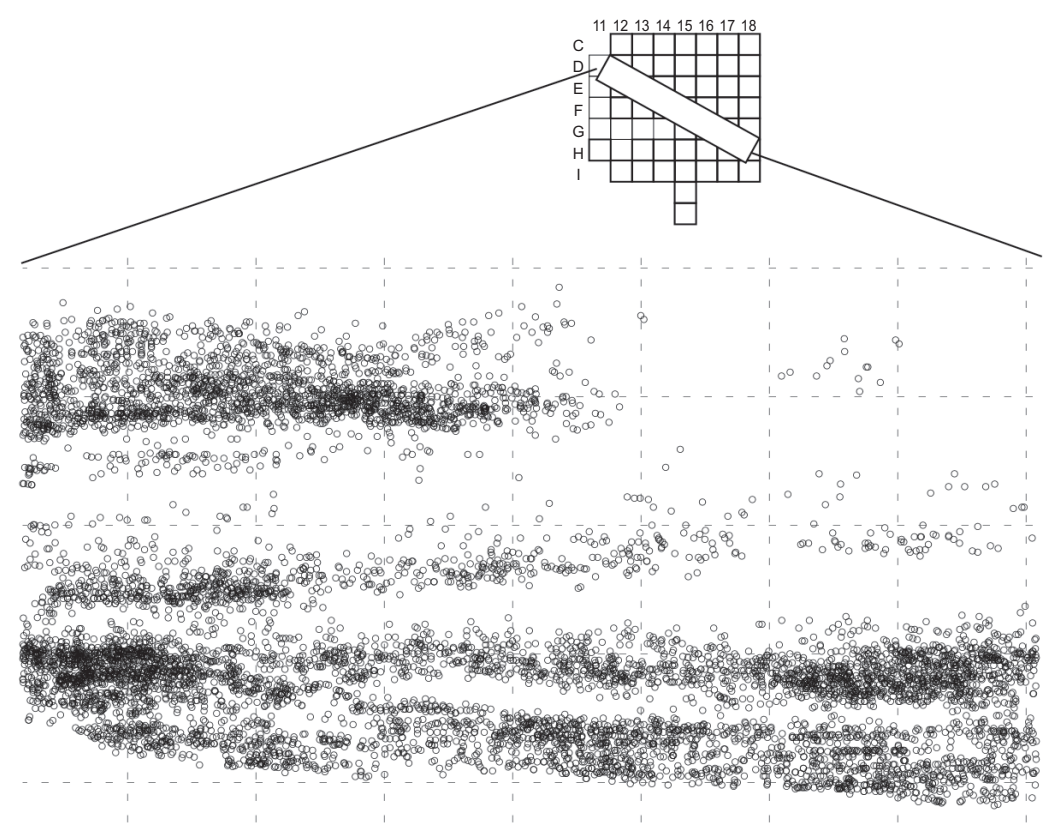

$\begin{array}{ll}0 \quad 2 m & a\end{array}$

Fig. 7 - a, diagramme de projection du matériel lithique montrant un pendage dans le sens de l'axe supposé de l'ancien réseau karstique; $\boldsymbol{b}$, localisation en plan des objets archéologiques roulés qui se répartissent aussi selon cet axe supposé (DAO: H. Dibble, Université de Pennsylvanie).

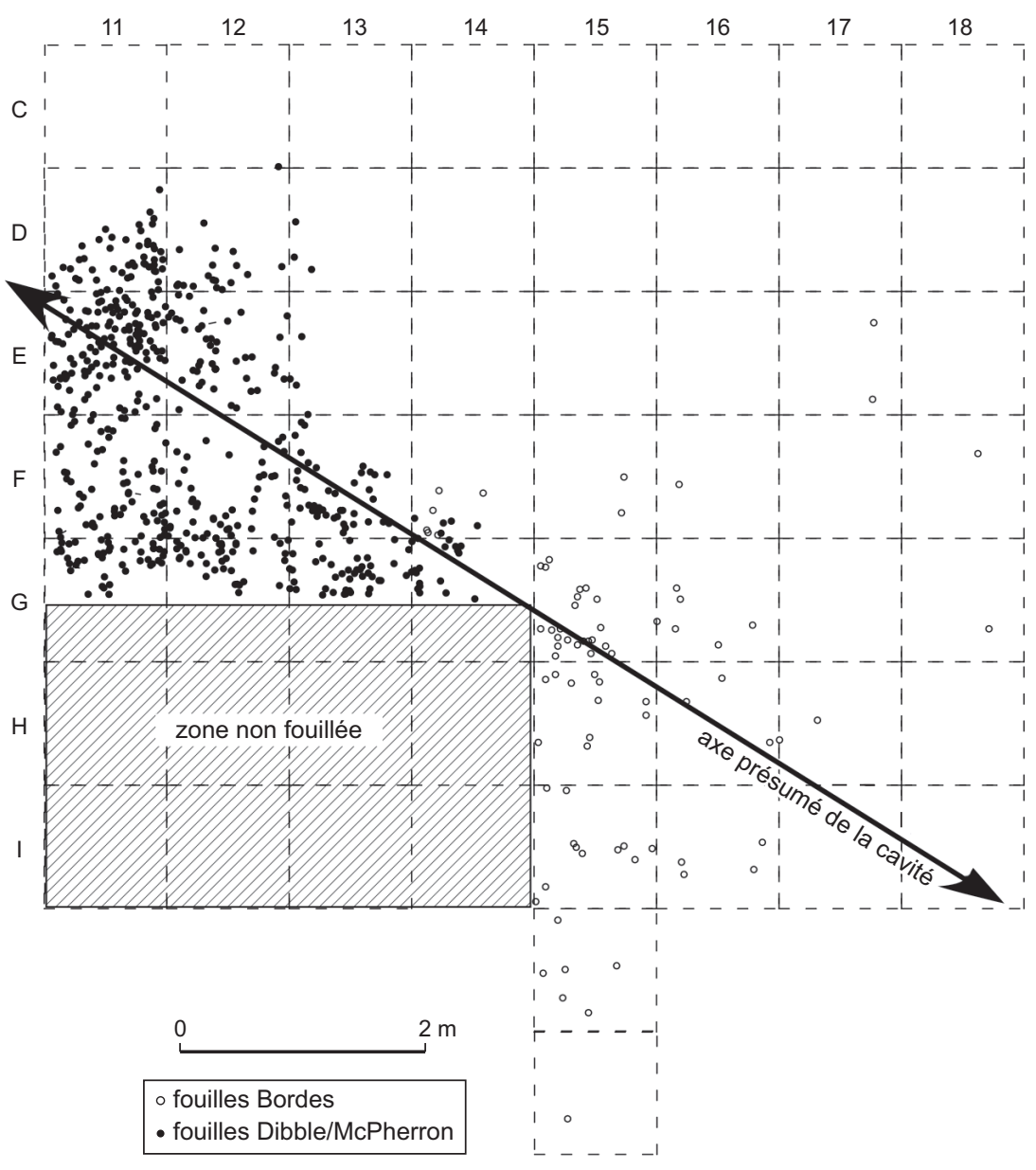

b 


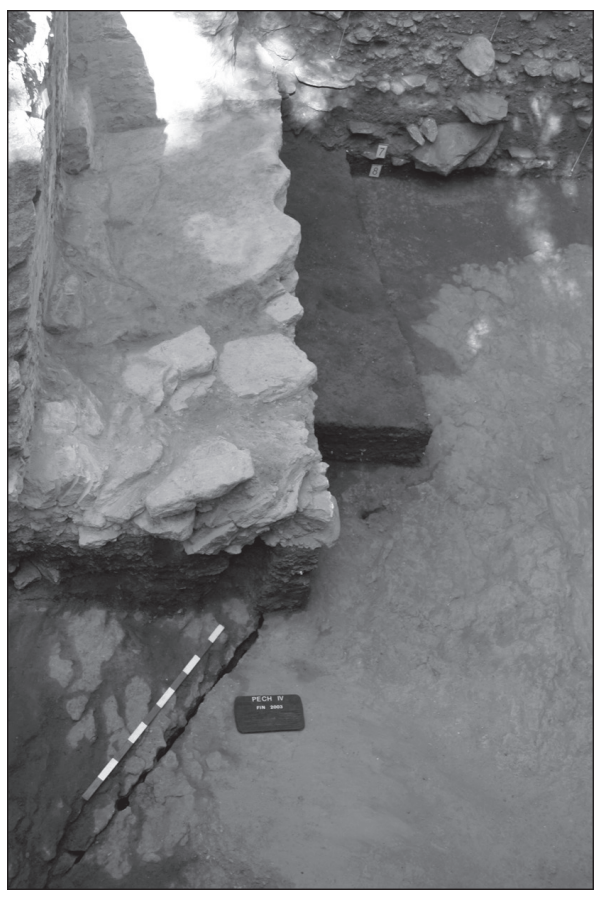

- Fig. 8 - Photographie de la microfaille observée dans le socle rocheux (cliché: P. Goldberg, Université de Boston).

- Fig. 9 - Photo et relevé de la coupe ouest, carrés H11-D11 (cliché et DAO: P. Goldberg, Université de Boston).

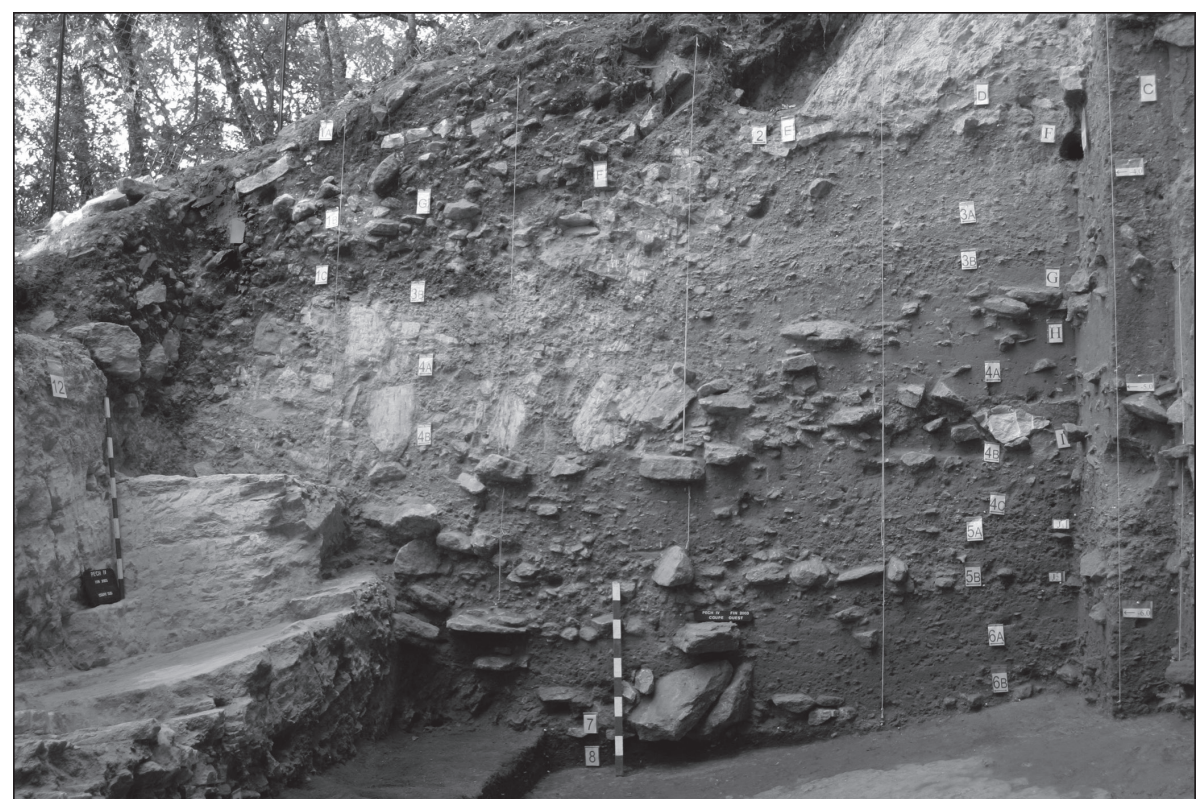

a

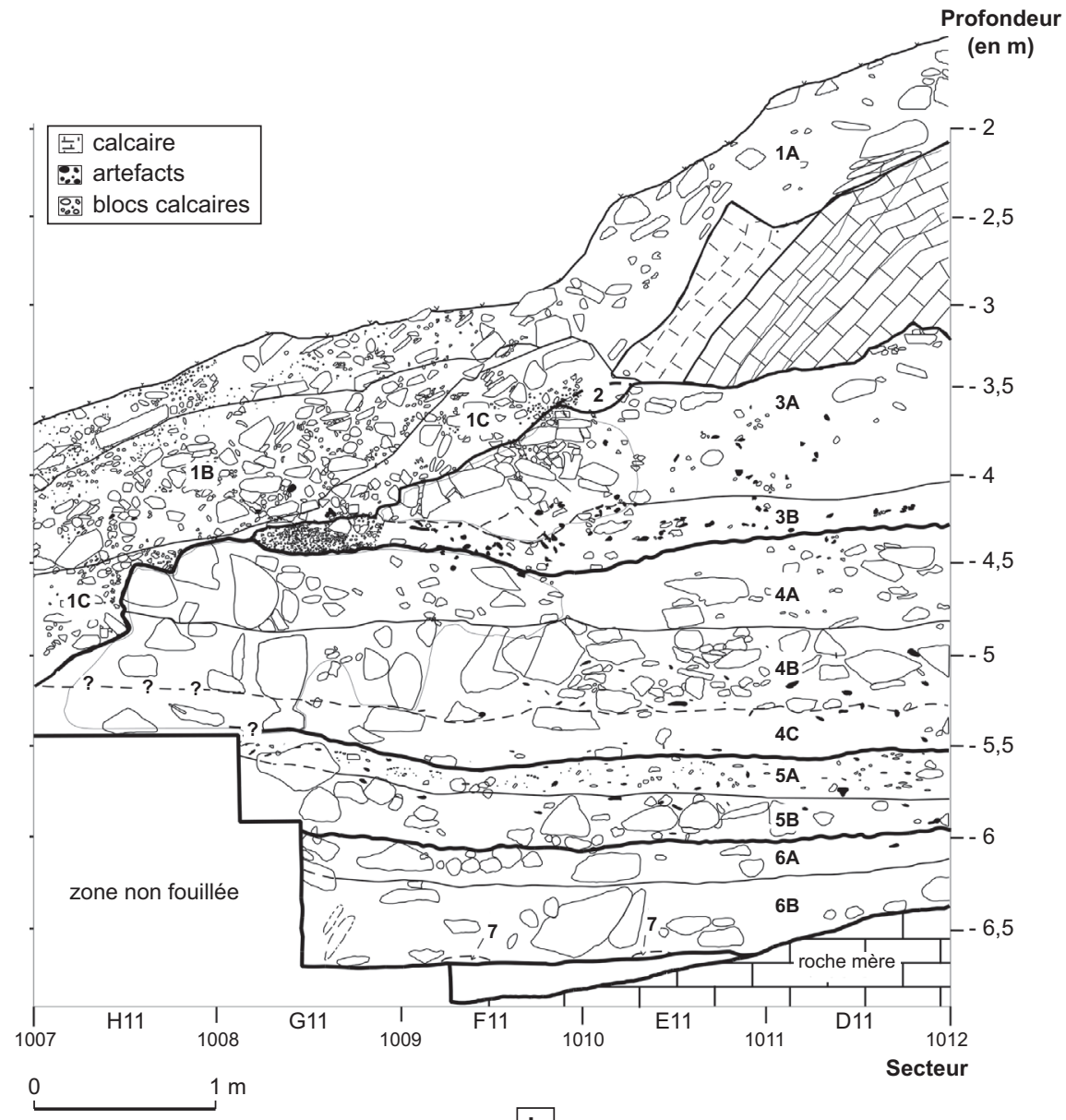

b 


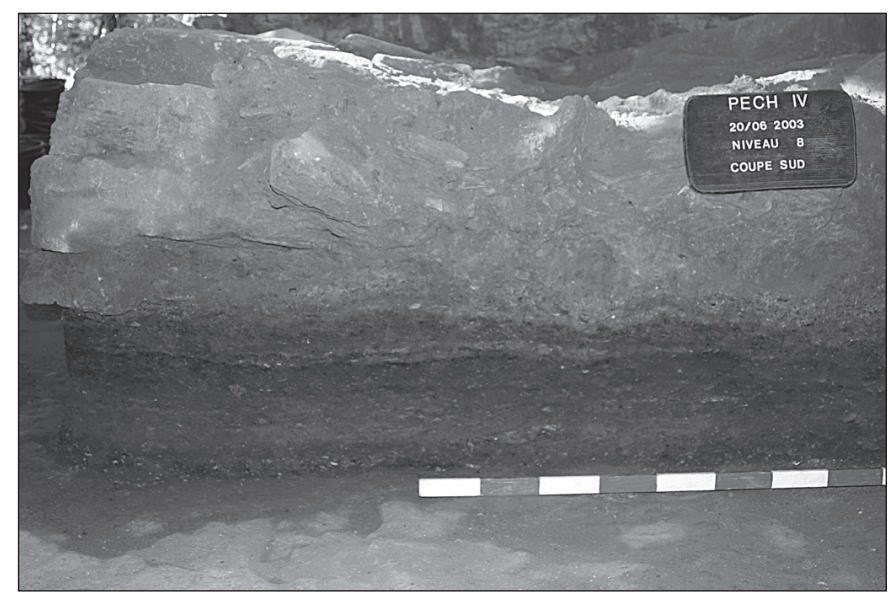

Fig. 10 - Coupe de la couche 8 renfermant des lentilles de cendres et de charbons (cliché: P. Goldberg, Université de Boston).

sont tombés, de l'intérieur vers l'extérieur. Les blocs anciens se retrouvent donc au nord et les plus récents au sud.

La couche 4 de $95 \mathrm{~cm}$ à $130 \mathrm{~cm}$ de puissance présente, dans une matrice sablonneuse rouge, de petits blocs d'effondrement du plafond. Elle est subdivisée en 4A, 4B et 4C. Concentrés dans les parties sud du site, ils sont plus fréquents dans la partie inférieure de 4B et 4C. Les parties sud de $4 \mathrm{~A}$ et $4 \mathrm{~B}$ ont été cimentées par le carbonate, probablement à cause du ruissellement de l'eau après la disparition du surplomb.

Entre les couches 4 et 3, on note une nouvelle lacune stratigraphique.

La couche 3 se distingue de la couche 4 par des sédiments plus compacts et cimentés renfermant moins de fragments calcaires. Ces derniers sont arrondis et semblent être quelque peu dissous. La matrice est rouge et sablonneuse, mais contient plus de calcite que dans la couche sous-jacente, sauf dans les sections les plus au nord. La partie inférieure de la couche (3B) s'enfonce ou repose avec un contact net sur la couche 4 . Ici, aussi, les parties sud sont plus cimentées que les parties nord en raison de la position de la ligne de gouttière.

La couche 2 est présente par endroits le long de la coupe ouest. C'est un ensemble de galets calcaires, de tailles et de formes variées, enrobés dans une matrice calcaire limoneuse à sablonneuse. Le contact entre les couches 2 et 3 semble dessiner une dépression (un bassin ou une tranchée), produite par l'eau cascadant de l'ancien sommet de la grotte. Ce réceptacle a été comblé par des gros blocs roulant du sommet et du versant, ainsi que par l'effondrement de la couche 3 . Ce phénomène pourrait expliquer la

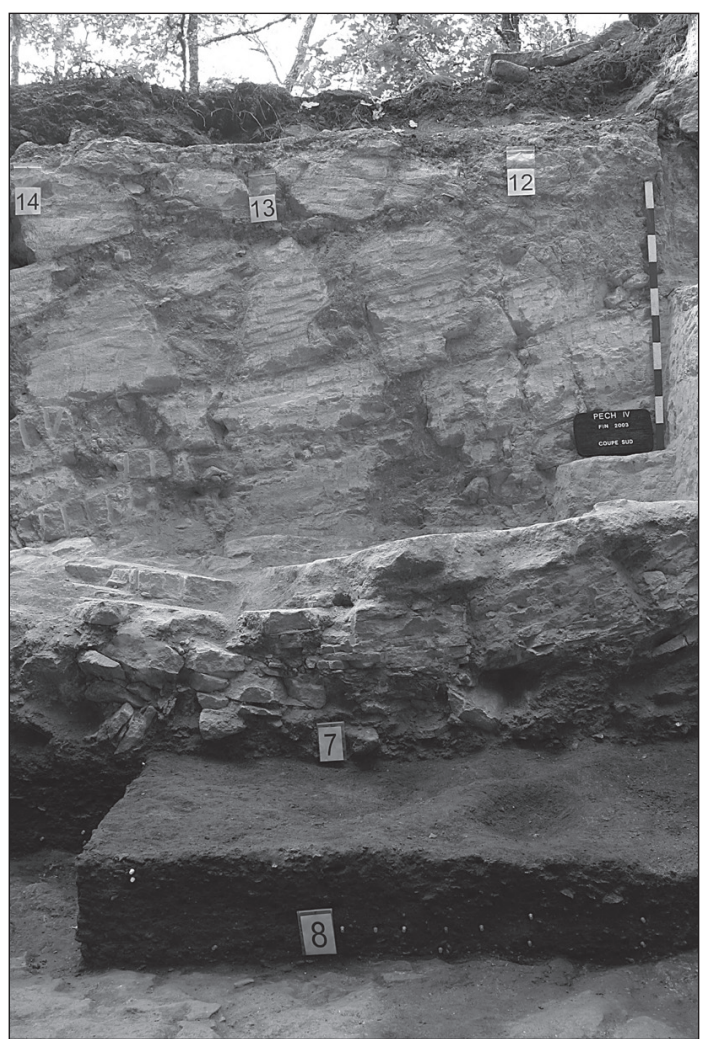

Fig. 11 - Couches $6 A$ et $6 B$ (effondrement de la voûte) sur la coupe sud au niveau des carrés H14-H12 (cliché: P. Goldberg, Université de Boston).

dispersion des objets lithiques retrouvés dans la couche 2 . La largeur de cette couche est d'environ un mètre et sa hauteur d'environ $75 \mathrm{~cm}$.

Entre les couches 2 et 1, nous avons affaire à une des phases principales de lacune stratigraphique et d'érosion.

La couche 1 résulte d'un lessivage du sol en pente et de dépôts dus à la chute du toit pendant les périodes historiques qui ont localement érodé quelques-uns des niveaux inférieurs. Elle contient des blocs calcaires mal classés dans une matrice limoneuse à sablonneuse riche en matière organique. En tant que partie du sol moderne, la couche 1 contient de nombreuses racines et des galeries de vers.

\section{RÉSULTATS MINÉRALOGIQUES ET MICROMORPHOLOGIQUES}

La micromorphologie des dépôts a révélé des caractéristiques à la fois géogéniques et anthropiques.

Dans toutes les couches, la majeure partie de la fraction grossière non anthropique est composée de fragments 
calcaires du substrat rocheux et de grains de quartz allant du limon au sable grossier. Les fragments calcaires sont souvent arrondis et la plupart ont des limites indistinctes ou des grains irréguliers. Des éléments naturels moins communs sont présents: grains de muscovite, glauconite, sable de carbonate, fragments de travertin et concrétions pédogénétiques d'oxyde de fer et de manganèse. La fraction fine géogénique est composée d'argiles et d'accumulations de calcite micritique secondaire ayant une structure cristalline biréfringente.

Les couches supérieures du site (1 à 5) diffèrent sensiblement des couches inférieures (6 à 8), en couleur, en pourcentage de matériel grossier et en abondance de fragments calcaires. Bien que certains échantillons contiennent des os brûlés, aucun ne possède de charbon, de fragments de cendres ou de matrice organique (quelques charbons étaient néanmoins visibles lors de la fouille de la couche 3 ). Les matériaux anthropiques sont essentiellement des fragments d'os isolés et vraisemblablement de rares morceaux de coquillages terrestres. Ces matériaux ont souvent la taille d'un grain de sable et sont subangulaires à arrondis.

Les couches 5 à 1 contiennent moins de matrice et moins de fragments de calcaire que les sous-jacentes. La distribution des fractions fines et grossières tient compte de ces différences: elle est chitonique à gefurique dans les couches supérieures (Stoops, 2003), et elle est porphyrique à gefurique dans les couches inférieures.

Dans les couches inférieures (6 à 8), les fractions fines et grossières contiennent de fortes proportions en matériel anthropique, y compris des os et des fragments de dents, de silex et des cendres, mais uniquement dans les couches 7 et 8 . Les os et les fragments de dents constituent la plus grande proportion de la fraction grossière anthropique. Ces fragments sont anguleux à arrondis, relativement petits, visibles tant sur le terrain qu'en lame mince. En moyenne, leur taille est comprise entre $0,25 \mathrm{~cm}$ et $2 \mathrm{~cm}$. Les os sont à la fois frais et brûlés, présentant une gamme de couleur allant du jaune crème au brun foncé, et une apparence blanchâtre pour les calcinés.

L'os brûlé est présent dans presque toutes les coupes des niveaux 7 et 8 . Dans la plupart des cas, il est mélangé de façon homogène avec l'os non brûlé, la stratification ou la séparation de ces deux types n'est généralement pas de mise. D'autre part, des bandes centimétriques de sédiments anthropiques de couleur sombre sont visibles tant sur le terrain que sur lame mince et correspondent à de grandes structures de combustion grossièrement circulaires comme celles fouillées en 2002. En dépit de cette morphologie, ces structures brûlées en bandes sont plus complexes que les structures de combustion découvertes dans les sites du Paléolithique moyen du Proche-Orient, tel que Kébara (Meignen et al., 1989 et 2007).

Plusieurs fragments d'os compact et spongieux de la couche 8 montrent in situ des cassures, dont des morceaux de forme angulaire peuvent être remontés sur le terrain. Ailleurs, les plus fragiles sont intacts. Ces fragments présentent également les traits caractéristiques d'une action chimique postdépositionnelle: beaucoup sont partiellement ou totalement tachetés par le fer. Les changements chimiques postdépositionnels qui ont produit ces «taches» ne semblent pas avoir affecté la préservation des fragments d'os, puisque ces derniers se sont maintenus pendant la fouille et les bords des grains restent distincts au microscope.

Le charbon de bois est présent dans les couches 7 et 8 . Les gros éléments sont rares et montrent occasionnellement une préservation de la structure cellulaire, telle que les vésicules, alors que les fragments plus petits sont généralement amorphes avec un diamètre inférieur à $0,25 \mathrm{~mm}$. Il est important de noter que pour le charbon de bois les gros fragments sont plus rares que pour les os brûlés.

Les cendres de bois des couches 7 et 8 sont présentes sous trois formes principales. Dans la première, elles sont bien conservées. Ce sont des couches presque pures d'environ $5 \mathrm{~mm}$ d'épaisseur contenant des grains de calcite rhomboédrique intacts. Très compactes et massives, elles sont visiblement feuilletées et cimentées par le carbonate micritique secondaire. Certaines couches cendreuses contiennent des vides d'entassement, alors que d'autres présentent des fissures, dissociées par endroits en fragments angulaires. Dans la seconde forme, il s'agit de morceaux isolés de strates cendreuses cimentées. Les fragments sont arrondis mais présentent encore des litages et sont souvent orientés de façon aléatoire, ce qui suggère, dans une certaine mesure, qu'ils ont été remaniés. La troisième forme se caractérise par une matrice meuble. Cette cendre contient également des grains rhomboédriques calcitiques, mais elle a un aspect plus poudreux en raison de l'incorporation d'autres matériaux, tels que des grains argileux et des fragments de charbon. Les cendres mélangées à la matrice recouvrent souvent de grands fragments de calcaire, le silex et l'os sous les couches de cendres intactes. Quelques échantillons contiennent des cendres mélangées à la matrice tout au long de la section. Les lentilles de cendres visibles sur les coupes sont composées de couches de cendres cimentées et de la matrice riche en cendres meubles. 
La matrice de la couche 7 a une couleur plus claire, ce qui donne à la couche 8 une apparence généralement plus sombre et plus organique sur le terrain. À l'échelle microscopique, la différence de couleur est due à la présence de fragments de charbon de granulométrie limoneuse mélangés à la matrice de la couche 8 . D'autre part, la matrice de la couche 7 ne contient que des oxydes de fer et des cendres. La base de la couche 8 est également plus claire que les parties intermédiaires et supérieures. Cette différence de couleur provient des concentrations plus élevées de carbonate secondaire poreux dans les sédiments qui recouvrent le substrat calcaire.

Dans l'ensemble de la séquence, les matériaux biogénétiques sont pratiquement absents, à l'exception d'un seul fragment de coprolithe observé dans une lame, juste audessus du socle rocheux.

\section{LES PROCESSUS DE FORMATION DU SITE}

Les lames minces, en particulier celles des couches supérieures, contiennent une fraction grossière qui reflète principalement l'origine géologique des sédiments, alors que la fraction fine, si elle est présente, correspond surtout à l'activité anthropique et/ou aux processus postdépositionnels de dissolution et de précipitation. Sur une base identique de granulométrie, de classe et d'état d'émoussement, il est évident que les sables quartzeux, ou carbonatés, et les fragments de calcaire proviennent de l'encaissant. La plupart des composants mineurs de la fraction grossière, tels que la glauconite et le mica, sont présents sous forme de traces dans le bed-rock, où ils apparaissent sous forme de grains frais ou dégradés; dans ce dernier cas, ils sont associés aux taches de fer de la calcite. De nombreux échantillons micromorphologiques montrent des fragments de calcaire activement dissous qui se distinguent par les bords ondulés des grains disparaissant localement dans le sédiment de la matrice. Quelques fragments calcaires se sont également dégradés de l'intérieur et les petites cavités sont remplies par du sédiment fin. Dans certains échantillons, le carbonate de ces fragments a été complètement dissous. Il est remplacé par des concentrations clastiques de sable de quartz, muscovite et glauconite. Par conséquent, la quasi-totalité des sédiments grossiers du site sont soit des dérivés locaux, soit générés sur place.

À l'exception des couches 7 et 8 , un élément de la fraction fine des sédiments est également issu de l'altération du calcaire et de la scission de ses composants insolubles. La couleur rougeâtre générale de la matrice provient des grains d'oxyde de fer et de manganèse de granulométrie limoneuse et argileuse. Ces grains sont aussi présents dans le substrat calcaire, mais il n'est pas certain que sa dissolution soit la seule et unique source du fer dans les sédiments. La plupart des grottes et des abris sous roche reçoivent du matériel provenant du manteau altéritique qui les recouvre: de fines particules qui s'infiltrent par les fissures de la roche. Une partie de la matrice se compose de minéraux argileux pouvant avoir la même origine, ou ayant pu se former sur place à partir de l'altération de minéraux grossiers, tels que la glauconite et la muscovite.

Beaucoup de lames minces contiennent aussi du matériel carbonaté fin, d'origine secondaire. Il s'accumule sous forme de taches avec une morphologie en tuf, ou dans les espaces vides avec des revêtements micritiques. De fortes concentrations cimentées peuvent aussi indiquer d'anciennes localisations de la ligne de gouttière. La dissolution active des fragments de calcaire et les accumulations de carbonate secondaire indiquent que l'environnement sédimentaire du Pech de l'Azé IV a été alternativement défavorable et propice à la préservation de la calcite primaire. Cette observation est particulièrement pertinente en ce qui concerne l'origine et la préservation des structures de combustion des couches 7 et 8 .

Les composants anthropiques et leur association fournissent une preuve évidente que la combustion est survenue au cours de la mise en place des dépôts des couches 8,7 et peut-être 6 . Cette combustion décelée par les lentilles de cendres intactes correspond probablement à la série de structures de combustion fouillées pendant la saison 2002. Les cendres sont principalement composées de calcite, ce qui indique qu'elles n'ont été sujettes à aucune diagenèse due au phosphate. L'étude minéralogique FTIR confirme également l'absence de phosphate minéral authigènétique dans ces sédiments. L'absence de diagenèse suggère que le site n'a pas contenu de source de phosphate, souvent présent sous la forme d'excréments d'animaux, comme le guano de chauve-souris. Contrairement au Pech de l'Azé II, le site n'a pas été occupé par des hyènes, et s'il a été occupé par des chauves-souris, leur présence n'a pas affecté les zones d'activités centrales (Goldberg, 1979). La présence de grains rhomboédriques dans les couches de cendres - essentiellement des calcites pseudomorphes provenant de l'oxalate de calcium présent à l'origine dans le bois indique également que les cendres sont bien conservées.

Nous interprétons la présence d'os cassés in situ comme une indication de piétinement, ce qui suggère que cela correspond à des zones fréquentes d'activités (l'entrée du 
site par exemple), par opposition aux régions périphériques (Schiegl et al., 2003). Une telle conclusion n'est pas surprenante compte tenu de l'emplacement de l'entrée de la grotte et l'accumulation des structures de combustion. Un piétinement intense peut aussi contribuer à la compacité des couches cendreuses et explique le mélange des os brûlés et non brûlés, des cendres et des charbons de bois. Toutefois, on ne peut pas exclure que ce dernier soit le résultat d'activités de nettoyage des foyers telles que la vidange ou la redistribution des matériaux brûlés avant un nouveau feu. La concentration d'os brûlés, de cendres et de charbon dans la couche 8, à l'intérieur de l'ancienne ligne de ruissellement, montre que les activités de combustion semblent avoir été limitées à ce secteur.

Les fissures présentes dans certaines couches de cendres montrent que ces dernières sont cimentées, un processus probablement produit juste après leur dépôt (Karkanas et al., 2000). Les couches 7 et 8 présentent également les caractéristiques associées à la dissolution du carbonate. Comme mentionné plus haut, de nombreux fragments de calcaire semblent avoir été activement dissous. De plus, les fragments de cendres cimentées contiennent souvent des sillons, caractéristique de l'épuisement en calcite (Bullock et al., 1985). Cependant, par rapport aux couches 5 à 1 , le carbonate des niveaux inférieurs est relativement bien préservé. Cette préservation peut provenir de la protection physique et de la capacité d'amortissement des blocs du toit de la couche $6 \mathrm{~B}$ contre l'action des eaux souterraines.

L'étude micromorphologique montrant que les sédiments sont le produit interne de la dissolution du substrat calcaire plaide en faveur de l'idée que le Pech de l'Azé IV est une grotte effondrée. De plus, un des échantillons micromorphologiques contient un morceau de travertin. À partir de là, nous pensons que Pech de l'Azé I, II et IV, à un moment donné de leur histoire, ont fait partie d'un même réseau karstique, système phréatique préquaternaire, qui suivait le bord de la colline. Progressivement, l'érosion des versants l'a détruit, colmaté ou caché. Au niveau de la coupe-témoin du Pech de l'Azé IV, probable exutoire d'un conduit, plusieurs épisodes de la chute du toit ont été enregistrés (par exemple les couches 6B, 5A, 4B, 4C et 1). Ces épisodes ont abouti à la migration vers le nord de la ligne de gouttière de la grotte. Ce phénomène est lisible en microstratigraphie. De petits dépôts de travertins et d'accumulations secondaires de calcite se sont déplacés en direction du fond de la grotte au fur et à mesure que l'on monte dans la stratigraphie. L'effondrement final du toit a entraîné la réduction du porche de quelques mètres.
Ce porche a été enlevé au cours des fouilles de F. Bordes, mais il reste visible sur la coupe-témoin ouest des fouilles actuelles.

F. Bordes a également observé les indices d'une altération postdépositionnelle des sédiments du Pech de l'Azé IV, en particulier le processus de cryoturbation. Ce dernier est identifiable au microscope sous la forme de petites masses de sédiments, compactes et arrondies, qui forment une microstructure granulaire ou désagrégée. D’un point de vue macroscopique, ce processus se marque par la présence de galets arrondis, l'inclinaison des artefacts, les mélanges et les lentilles de glace, autant d'éléments observés dans le gisement proche du Pech de l'Azé II (Goldberg, 1979; Texier, 2006). Au Pech de l'Azé IV, s'il existe des fragments de calcaire émoussés, les échantillons micromorphologiques associés ne révèlent pas d'agglomération des sédiments à grain fin. Ainsi, les blocs de calcaire arrondis, retrouvés dans la couche 6 , ne l'ont pas été in situ par l'action du gel-dégel, mais pourraient être des clastes hérités des processus phréatiques non périglaciaires de la période préquaternaire. La couche 5 présente une répartition des blocs calcaires qui suggère un processus de solifluxion, ou un mouvement en pente douce d'eau chargée de sédiments sous des conditions climatiques froides. Cependant, les lames minces ne montrent pas de microstructures ou de structures attestant ce fait.

\section{SÉQUENCE DE FORMATION DU SITE}

Lorsque l'occupation du Pech de l'Azé IV a commencé, la morphologie du site correspondait davantage à une grotte qu'à un abri sous roche. Le premier dépôt est de nature anthropique; l'activité liée à l'utilisation du feu a eu lieu directement sur la surface du socle rocheux, juste sous le porche existant à cette époque. Cette activité a inclus le dépôt de matériaux anthropiques mélangés aux sédiments de la grotte et à l'organisation des structures de combustion. La plupart des cendres sont présentes sous forme de lentilles et d'amas de grains, ce qui implique une chronologie intéressante des événements. Vraisemblablement, les cendres se sont accumulées en couches fines correspondant à des foyers occasionnels ou à des actes isolés de vidange des foyers, associés au piétinement. Immédiatement après le dépôt, le tassement gravitationnel a généré quelques cendres meubles qui ont filtré à travers le profil du sédiment et se sont fixées principalement sur les parties supérieures des gros grains. Les autres couches de cendres ont été rapidement cimentées par l'action de l'eau, par le ruissellement 
depuis le porche, et par les précipitations déviées par le vent à l'intérieur de la grotte. Après la cimentation, les couches cendreuses ont été brisées par le piétinement, et certains des sédiments se sont mélangés, peut-être au cours des activités de vidange des foyers qui ont suivi. Les fragments de cendres ont ensuite été soumis à la dissolution de la calcite, comme en témoignent les fissures et quelques fragments très poreux.

Le premier effondrement important du toit (couche 6B) a généré des blocs de calcaire massifs et plats qui ont protégé les structures de combustion des nouveaux piétinements et ont agi comme un tampon chimique. L'eau, en le traversant, s'est saturée en carbonate et a localement cimenté le bas de la couche 8 lorsqu'elle a atteint le sol rocheux de la cavité, l'indurant avec la calcite cristallisée (le spath). Cet épisode d'effondrement du toit a vraisemblablement changé la morphologie de la grotte et lui a donné une configuration plus ouverte: les sédiments, immédiatement au-dessus des blocs de calcaire, contiennent des concrétions de fer et de quartz d'origine pédogénétique. Les activités de combustion ont peut-être continué encore quelque temps ou ont été déplacées, comme pourraient le suggérer les os brûlés de la couche 6 trouvés dans la coupe est. Cependant, les sédiments résultants des structures de combustion n'ont pas été protégés de la dissolution du carbonate, et les seuls vestiges sont des os brûlés et des petits fragments occasionnels de charbon. On observe aussi une accumulation de sable non calcaire provenant d'une source sédimentaire interne, probablement des restes de remplissages endokarstiques, comme dans le cas de Pech de l'Azé II.

La couche $6 \mathrm{~A}$ a subi une érosion marquée dans les carrés nord (D11 à D14, E11 à E14, F11 à F14), suivie d'un remplissage de sable caillouteux.

La forte inclinaison de la couche 5 combinée à l'orientation chaotique des pierres et à l'épaississement de la couche 5B dans le carré G11, suggèrent un lobe possible de solifluxion s'amoncelant contre les éléments de la chute du toit, bien que de telles caractéristiques ne soient pas visibles au microscope. Cette phase (couche 5) marque le dernier épisode majeur de l'effondrement des blocs à l'entrée (carrés I, J et K) et le début d'un type différent d'effondrement: le retrait du porche vers le nord. À ce moment, la morphologie du site ressemble plus à un abri sous roche qu'à une grotte, tout comme F. Bordes l'avait observé.

L'argile limoneuse brun rougeâtre s'est accumulée dans la partie nord de la section, tandis que les blocs imbriqués du toit marquent le retrait du porche vers le sud. Les zones cimentées du sédiment représentent les positions des anciennes lignes d'égouttement, qui se sont déplacées vers le nord à chaque épisode d'effondrement.

La troncature et l'érosion de la couche 4A par du sable angulaire caillouteux, avec un contact net, indiquent la possibilité d'une cryoclastie. Ces dépôts de climat froid sont tronqués latéralement par un chenal qui s'est formé par l'écoulement de l'eau des lignes de gouttière existant alors. Le dépôt de la couche 2 a rempli ce chenal avec des morceaux centimétriques de substrat calcaire, composés de blocs angulaires et de galets émoussés issus du retrait du toit et de l'effondrement de la pente de la colline adjacente. Cette couche contient également quelques matériaux retravaillés de la couche 3 qui se sont écroulés des bords du canal. L'effondrement final du toit s'est produit au cours des temps historiques, comme le montre la rotation des blocs du toit dans le carré D11. L'érosion finale du coteau et l'accumulation de colluvions ont enterré le site sous des pierres centimétriques dans un sable argileux organique brun foncé.

Nous notons enfin que certains des processus post et syndépositionnels peuvent faire la lumière sur la détérioration des bords constatée sur les objets lithiques. La couche 7 par exemple, constituée de silex très abrasés et roulés dans une matrice sableuse, résulte normalement de la cryoturbation ou de la solifluxion. Malheureusement, suite à la déformation due aux chutes de pierres, il est difficile de déterminer si la couche était d'épaisseur uniforme ou variable, et si elle était sous la forme d'un lobe de sédiment qui aurait coulé $\mathrm{du}$ fond de la grotte; l'absence de pendage ne contribue pas non plus à déterminer l'origine exacte de cette unité. En tous cas, tout ceci fait penser à la couche 5 déformée et cryoturbée du Pech de l'Azé II (Laville et al., 1980).

La situation est assez semblable pour le niveau 5B. À l'arrière de la grotte (D, E, F), les couches reposent de façon non concordante sur la couche 6 avec un contact net, mais ondulé et irrégulier. Elles sont composées de lithoclastes de calcaire arrondis et de silex aux arêtes endommagées. Vers l'ouest, ces dépôts se juxtaposent et recouvrent les grands blocs localisés en G, H, I et J. Ces derniers correspondent au délitage de l'auvent rocheux au sud de la ligne $\mathrm{H}$ qui protégeait le site. De ces relations stratigraphiques, il semble que la couche $5 \mathrm{~B}$ (et 5A) est légèrement postérieure à la chute du toit, et représente une période majeure de la solifluxion/cryoturbation des sédiments provenant de l'intérieur de la grotte, comme le montre l'émoussement des clastes et leur juxtaposition contre les blocs. Une telle situation peut être suggérée pour le contact 3B/4, qui est net, et dans lequel la couche $3 \mathrm{~B}$ est riche en éboulis angulaires dérivant également de l'intérieur de la grotte. 


\section{LES PROCESSUS DE FORMATION DU SITE À PARTIR DU REGISTRE ARGHÉOLOGIQUE}

Si l'évaluation des processus de formation du gisement peut être abordée par la géologie et l'archéozoologie, il est intéressant de prendre en compte les données issues du seul registre archéologique. Pour les objets lithiques, on peut observer leur état de conservation, la composition et la fragmentation, leur état de fraîcheur (usure des bords, des arêtes) et analyser les mesures de fabrique.

\section{Orientations des objets (analyse des fabriques)}

Il est désormais reconnu qu'une évaluation des orientations des clastes constitue une bonne estimation du processus de formation d'un dépôt (Lenoble, Bertran, 2004; McPherron, 2005). Cette notion est basée sur la modélisation des orientations qui indique le type et l'intensité de l'altération postdépositionnelle. Comme évoqué plus haut, l'enregistrement des vestiges a été réalisé à Pech de l'Azé IV avec une station totale. Nous sommes désormais capables d'intégrer nos données à un large référentiel comparatif provenant d'un bon nombre de sites paléolithiques issus de différents contextes. En complément, les mesures concernant l'orientation sont analysées suivant les méthodes préconisées par Lenoble et Bertran (2004), afin de pouvoir comparer nos données géologiques et archéologiques avec leur référentiel expérimental.

L'agencement des objets est évalué par la double observation de l'orientation (angulation mesurée horizontalement selon le grand axe de l'objet par rapport à un nord géographique ou arbitraire) et de l'inclinaison (mesure d'angle prise cette fois sur un plan vertical du grand axe par rapport à un plan horizontal). Un report sphérique (diagramme de Schmidt) et des diagrammes circulaires (diagramme de Rose) permettent de visualiser les résultats. D.I. Benn (1994) a montré que la configuration des indices calculés à partir de valeurs informatisées (Watson, 1965; Woodcock, 1977), et reportés sur un diagramme triangulaire, peut être utilisée pour distinguer l'orientation des mesures de fabrique, et pour présenter des données issues d'un référentiel expérimental et de contextes archéologiques (Bertran et al., 1997; Lenoble, Bertran, 2004).

Le test d'uniformité de Rayleigh (tabl. II) indique que la plupart des niveaux montrent une orientation préférentielle des artefacts et un pendage sélectif. Les diagrammes de Rose et Schmidt représentés dans la fig. 12 indiquent que dans ces cas, les artefacts tendent à se regrouper dans une
Tabl. II - Moyennes des angles d'inclinaison et d'orientation par couche. Le nombre d'objets, la moyenne, la déviation standard (DS) et le test d'uniformité de Rayleigh sont indiqués pour chacune d'elles. Les valeurs du test de Rayleigh inférieures à.05 indiquent que les angles ne sont pas distribués de façon uniforme.

\begin{tabular}{|c|c|c|c|c|c|c|c|c|}
\hline \multirow{2}{*}{ Couche } & \multirow{2}{*}{ Nombre } & \multicolumn{3}{|c|}{ Orientation } & \multicolumn{3}{c|}{ Inclinaison } & \multirow{2}{*}{ L } \\
\cline { 3 - 8 } & & moyenne & DS & Rayleigh & moyenne & DS & Rayleigh & \\
\hline 1B & 127 & 150,71 & 90,40 & $\mathbf{0 , 0 0}$ & 22,30 & 18,56 & 0,00 & 0,44 \\
\hline 1C & 26 & 149,54 & 86,04 & 0,06 & 23,74 & 25,29 & 0,00 & 0,42 \\
\hline 1D & 21 & 203,22 & 112,06 & 0,64 & 31,21 & 21,30 & 0,00 & 0,48 \\
\hline 2 & 40 & 48,23 & 107,46 & 0,31 & 26,55 & 20,14 & 0,00 & 0,42 \\
\hline 3A & 324 & 166,78 & 107,46 & $\mathbf{0 , 0 0}$ & 24,50 & 23,44 & 0,00 & 0,39 \\
\hline 3B & 591 & 199,82 & 119,42 & $\mathbf{0 , 0 0}$ & 13,61 & 17,19 & 0,00 & 0,24 \\
\hline 4A & 312 & 174,21 & 108,63 & $\mathbf{0 , 0 0}$ & 11,43 & 15,32 & 0,00 & 0,23 \\
\hline 4B & 118 & 171,72 & 115,57 & 0,13 & 13,99 & 15,26 & 0,00 & 0,24 \\
\hline 4C & 1147 & 155,18 & 107,79 & $\mathbf{0 , 0 0}$ & 8,70 & 9,30 & 0,00 & 0,22 \\
\hline 5A & 772 & 173,97 & 138,72 & 0,11 & 9,21 & 10,57 & 0,00 & 0,15 \\
\hline 5B & 219 & 89,58 & 136,97 & 0,49 & 15,11 & 17,57 & 0,00 & 0,22 \\
\hline 6A & 1228 & 151,49 & 111,23 & $\mathbf{0 , 0 0}$ & 11,82 & 13,09 & 0,00 & 0,24 \\
\hline 6B & 1110 & 196,85 & 105,90 & $\mathbf{0 , 0 0}$ & 8,35 & 9,33 & 0,00 & 0,22 \\
\hline 7 & 52 & 115,38 & 81,46 & $\mathbf{0 , 0 0}$ & 12,63 & 15,69 & 0,00 & 0,40 \\
\hline $\mathbf{8}$ & 493 & 154,18 & 97,33 & $\mathbf{0 , 0 0}$ & 7,59 & 9,48 & 0,00 & 0,26 \\
\hline
\end{tabular}

partie du graphique, ils sont alors unimodaux. Les orientations moyennes des niveaux significatifs sont d'environ 164 degrés, ce qui correspond assez bien à l'orientation suggérée par le système karstique dont le Pech de l'Azé IV faisait partie (fig. 13).

Le diagramme de Benn expose la variabilité des couches dans l'échantillon correspondant à ce qui était déjà connu sur ces niveaux essentiellement par la géologie mais également par des mesures telles que l'altération des bords des artefacts (fig. 14). La plupart des niveaux s'inscrivent dans le plan, suggérant une faiblesse d'alignements postdépositionnels. Plusieurs des niveaux se placent vers le centre du diagramme révélant des taux élevés d'isotropie. Les niveaux 1B, 1C et 1D sont des dépôts holocènes qui recouvrent les principaux dépôts archéologiques et sont clairement des coulées de versant. Sur le diagramme de Benn, ils sont comparables aux données publiées sur les coulées de débris (Benn, 1994; Lenoble, Bertran, 2004). Le niveau 2, qui s'inscrit près du centre du graphique, est une petite poche de matériel d'origine indéterminée qui semble géologiquement remanié. Les niveaux 3A et 3B sont intéressants car sédimentologiquement similaires, mais ils sont localisés graphiquement de façon très différente. La distinction entre ces deux niveaux se base principalement sur l'augmentation de la densité en graviers et en artefacts lithiques dans la couche 3B d'environ $15 \mathrm{~cm}$ d'épaisseur à la base du dépôt. 


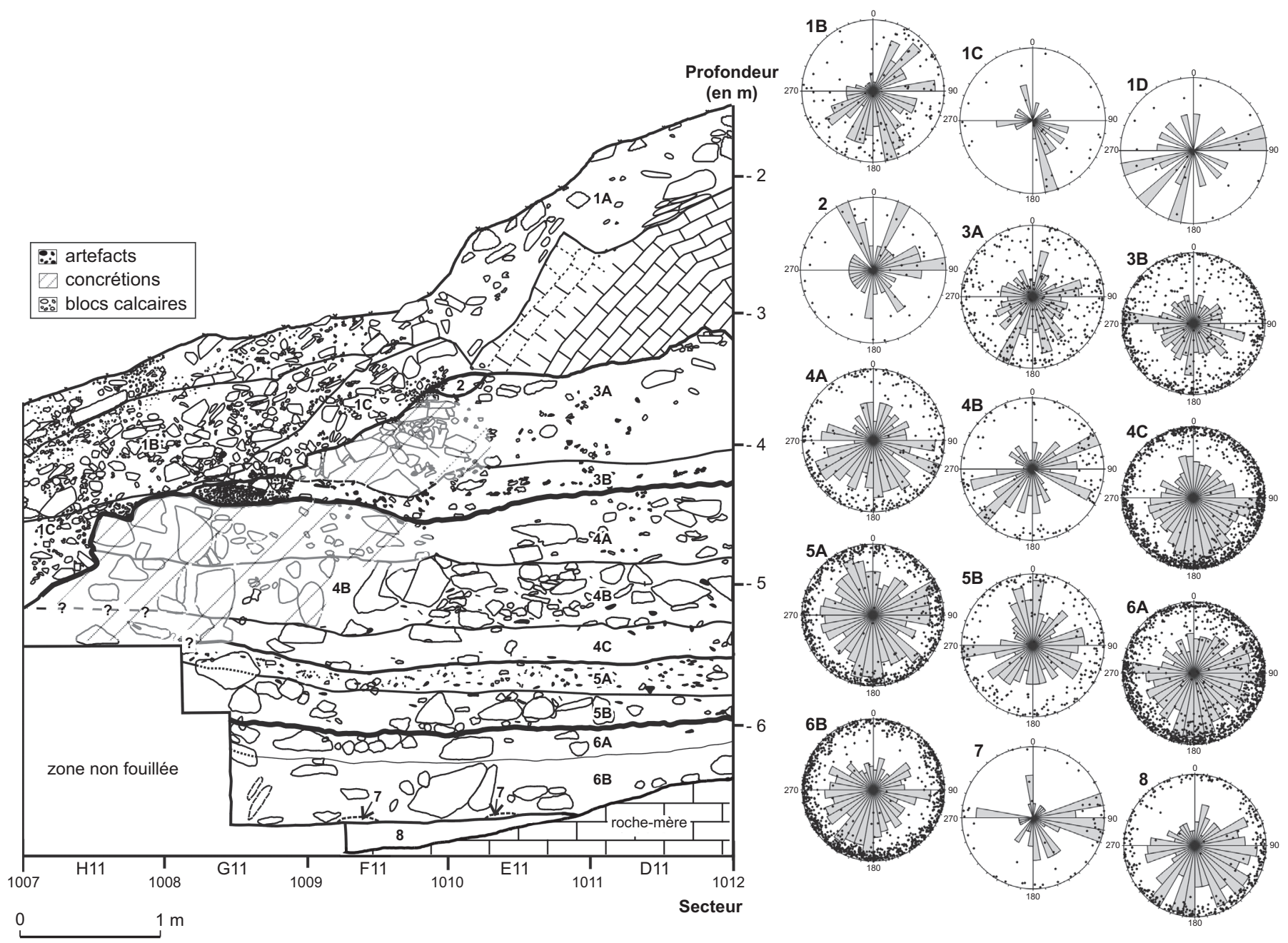

Fig. 12 - Orientation des objets (fabriques) par couche (DAO: S. McPherron, Max Planck Institute).

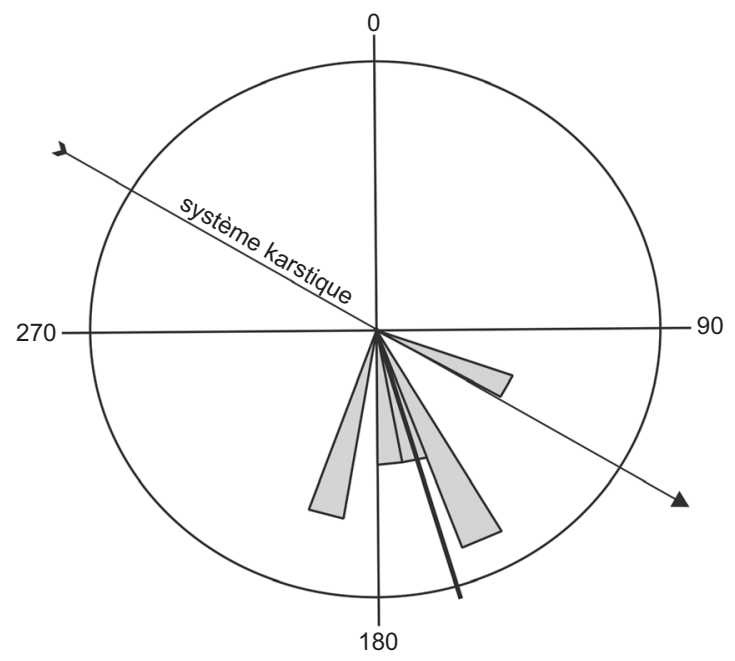

Fig. 13 - Axes privilégiés d'orientation des objets par rapport à celui du karst (DAO: S. McPherron, Max Planck Institute).

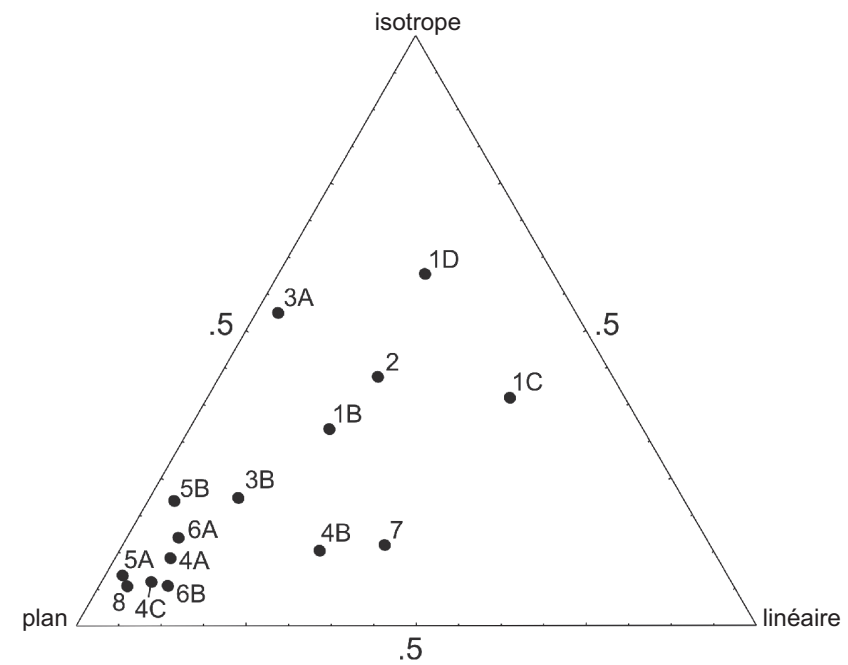

Fig. 14 - Diagramme de Benn pour l'ensemble des couches (DAO: S. McPherron, Max Planck Institute). 
La transition de ce dépôt avec celui de $75 \mathrm{~cm}$ d'épaisseur du niveau 3A est graduelle; les données sur l'orientation appuient clairement cette double division (McPherron et al., 2005). Le niveau 3A est beaucoup plus isotrope que 3B. Ce point est clair par rapport aux valeurs moyennes du pendage qui sont près de deux fois celles du niveau 3B, et les plus élevées parmi les niveaux archéologiques pléistocènes. Le niveau 3B serait également plus proche du plan si seuls le carré D11 et une partie de E11 avaient été inclus dans l'analyse. En effet, il y a un pendage dans les dépôts des carrés F11/E11 qui augmente l'isotropie ${ }^{3}$.

Les niveaux $4 \mathrm{~B}$ et 7 , non holocènes, ont les plus petits échantillons, ce qui peut affecter leurs caractéristiques. La taille de l'échantillon du niveau 7 provient de son contexte sédimentaire plus que de sa densité en artefacts. En effet, il s'agit d'un des niveaux du site le plus riche, les objets étant disposés les uns sur les autres dans une matrice très meuble. Celle-ci rend très difficile la fouille des artefacts sans en modifier l'orientation. De plus, la plupart des artefacts présentaient des bords fortement endommagés et étaient cassés en morceaux angulaires sans orientation claire. Cette donnée correspond aux observations géologiques qui plaident en faveur d'un niveau ayant subi une importante altération postdépositionnelle: la cryoturbation étant le facteur le plus probable (voir supra, p. 19). Les orientations des artefacts, dans ce cas, sont compatibles avec la solifluxion et une coulée de débris (Hills, 1972; Benn, 1994).

Sur la base des faits géologiques, le niveau 5B présente également des preuves de solifluxion. Cependant, dans ce cas, les orientations des artefacts n'en rendent pas compte. Un examen plus attentif de la distribution verticale des objets endommagés de ce niveau a montré que sa partie inférieure avait été plus touchée que sa partie supérieure. Des travaux sont actuellement en cours pour diviser cette unité en deux afin que leurs caractéristiques, y compris leurs orientations, puissent être analysées séparément. Enfin, le niveau 4B se distingue des niveaux au-dessus et en dessous par la grande quantité de blocs d'effondrement qu'il contient. Cette différence pourrait expliquer la séparation graphique du niveau 4B des niveaux $4 \mathrm{~A}$ et $4 \mathrm{C}$, bien que l'hypothèse des blocs d'effondrement rendant des artefacts plus linéaires dans leur orientation ne soit pas claire. Il se peut que la linéarité suive l'orientation ou le pendage des blocs d'effondrement.

3. Dans ce type d'analyse, il faut être conscient des changements horizontaux dans les unités de dépôt. Ce type d'analyse peut justement être utilisé pour détecter ces changements au sein d'une unité.
En résumé, les données sur les orientations du Pech de l'Azé IV montrent des exemples d'interniveaux qui, à l'exception du niveau 5B, concordent assez bien avec d'autres sources d'information pour les processus de formation du site. Les dépôts holocènes sont distincts des pléistocènes dans la grotte et les subdivisions géologiques des couches sont confirmées par les données des orientations. Ces données archéologiques dérivées correspondent assez bien avec des données géologiques déjà publiées par Lenoble et Bertran (2004), et indiquent que la plupart des niveaux du Pech de l'Azé IV présentent des altérations postdépositionnelles minimes.

\section{État de conservation et de fracturation}

Le degré d'intégrité (état de conservation des bords et des arêtes) et de fracturation de chaque artefact lithique supérieur à $25 \mathrm{~mm}$ est systématiquement pris en compte. Ces données renseignent sur les facteurs de déformation postdépositionnelle qui peuvent être d'origine naturelle (cryoturbation) ou comportementale (piétinement) (McBrearty et al., 1998). Ces informations sont également intéressantes pour l'étude technotypologique (pseudooutils, denticulés naturels, etc.)

Nous distinguons quatre catégories de détérioration des bords ou des arêtes: objet intact, un bord détérioré, deux bords détériorés, objet roulé. Pour la fracturation, nous avons comparé le nombre d'éclats complets avec le nombre de fragments proximaux afin d'éviter d'augmenter artificiellement le pourcentage de pièces fracturées en comptant deux fois le même éclat cassé en un fragment proximal et en un fragment distal.

De façon générale, les couches du Pech de l'Azé IV ont produit entre $20 \%$ et $30 \%$ d'objets aux bords endommagés (tabl. III). Deux niveaux, 5B et 7, montrent cependant des concentrations très élevées des dommages de bord. Comme nous l'avons vu à la page 20, les couches $5 \mathrm{~B}$ et 7 ont déjà été identifiées comme ayant subi d'importantes altérations postdépositionnelles. Les niveaux $6 \mathrm{~B}$ et $6 \mathrm{C}$, souvent difficiles à distinguer de la couche 7 , ont également produit un nombre élevé d'objets aux bords endommagés par rapport aux autres couches, même si $6 \mathrm{C}$ présente un très petit échantillon.

Les résultats de la fracturation suivent plus ou moins les mêmes modèles (tabl. IV). Le niveau 7 a un taux très élevé de fracturation, comme les niveaux $6 \mathrm{~A}$ et $6 \mathrm{~B}$, probablement pour les mêmes raisons. En général, les niveaux inférieurs, y compris le niveau 8, ont des taux plus élevés de 
Tabl. III - État de conservation des objets lithiques par couche.

\begin{tabular}{|c|c|c|c|c|c|}
\hline Couche & Nombre & Objet intact & $\begin{array}{c}\mathbf{1} \text { bord } \\
\text { détérioré }\end{array}$ & $\begin{array}{c}\mathbf{2} \text { bords } \\
\text { détériorés }\end{array}$ & Objet roulé \\
\hline 3A & 1079 & 78,1 & 16,0 & 5,7 & 0,1 \\
\hline 3B & 1826 & 71,0 & 21,4 & 7,6 & 0,0 \\
\hline 4A & 130 & 69,2 & 17,7 & 13,1 & 0,0 \\
\hline 4B & 31 & 71,0 & 22,6 & 6,5 & 0,0 \\
\hline 4C & 399 & 68,9 & 24,3 & 6,8 & 0,0 \\
\hline 5A & 903 & 66,9 & 25,8 & 7,2 & 0,1 \\
\hline 5B & 355 & 40,0 & 30,4 & 22,8 & 6,8 \\
\hline 6A & 1308 & 67,4 & 22,8 & 9,6 & 0,2 \\
\hline 6B & 1181 & 48,0 & 31,0 & 19,6 & 1,4 \\
\hline 6C & 8 & 25,0 & 25,0 & 50,0 & 0,0 \\
\hline 7 & 1845 & 17,6 & 22,9 & 44,6 & 14,9 \\
\hline $\mathbf{8}$ & 1258 & 72,1 & 20,1 & 6,6 & 1,2 \\
\hline
\end{tabular}

Tabl. IV - État de fracturation des objets lithiques par couche.

\begin{tabular}{|c|c|c|c|c|}
\hline \multirow{2}{*}{ Couche } & \multicolumn{2}{|c|}{ Éclat entier } & \multicolumn{2}{c|}{ Fragments proximaux } \\
\cline { 2 - 5 } & nombre & pourcentage & nombre & pourcentage \\
\hline 3A & 828 & 75,2 & 273 & 24,8 \\
\hline 3B & 1344 & 72,3 & 516 & 27,7 \\
\hline 4A & 98 & 74,2 & 34 & 25,8 \\
\hline 4B & 26 & 83,9 & 5 & 16,1 \\
\hline 4C & 282 & 69,3 & 125 & 30,7 \\
\hline 5A & 654 & 70,9 & 268 & 29,1 \\
\hline 5B & 228 & 62,6 & 136 & 37,4 \\
\hline 6A & 879 & 63,8 & 499 & 36,2 \\
\hline 6B & 718 & 57,7 & 526 & 42,3 \\
\hline 6C & 3 & 37,5 & 5 & 62,5 \\
\hline 7 & 841 & 42,5 & 1136 & 57,5 \\
\hline 8 & 803 & 58,1 & 578 & 41,9 \\
\hline
\end{tabular}

fracturation. Dans ce cas, cette tendance est probablement liée à la combustion. Les niveaux 8 à $6 \mathrm{~A}$ ont le pourcentage le plus élevé en silex chauffés alors qu'il diminue considérablement pour les niveaux suivants.

Ainsi, prises dans leur ensemble, les indications apportées par l'orientation des artefacts, la détérioration des bords et la fracturation soutiennent les observations géologiques qui suggèrent une altération postdépositionnelle peu importante de ces dépôts, à l'exception du niveau 7 et d'une partie du niveau 5B. Pour le niveau 8, cette observation est appuyée par la présence de structures intactes de combustion, qui indique une altération minime en dehors du piétinement possible.

\section{PROPOSITION DE CORRÉLATION ENTRE DEUX SÉQUENCES STRATIGRAPHIQUES}

La lecture de la séquence stratigraphique mise au jour au cours de nos travaux a été réalisée de façon complètement indépendante de l'interprétation avancée par F. Bordes (1975). Ainsi, la question de la correspondance entre les deux séquences stratigraphiques, celle de F. Bordes et la nôtre, demeure posée. Il nous reste également à évaluer à quel point notre collection correspond à celle collectée par F. Bordes (McPherron, Dibble, 2000).

\section{DONNÉES STRATIGRAPHIQUES}

Nous disposons uniquement de la note préliminaire avec une description très sommaire des couches pour les fouilles de F. Bordes, ce qui n'a pas facilité notre approche.

Les deux fouilles ont atteint la roche-mère et des plages rubéfiées ont été observées. Le tableau $\mathrm{V}$ donne une synthèse des corrélations entre les deux séquences stratigraphiques prenant en compte les éléments de géologie (principales phases d'effondrement, surface d'érosion) et les données issues des industries (état du matériel archéologique, corrélations des principales couches archéologiques)

Bien que nous soyons en présence d'une stratigraphie de dépôts de pente, deux grandes phases d'éboulement facilement repérables ont été identifiées par les deux équipes: l'éboulis entre les couches X et J3C de F. Bordes et la couche 6 de $\mathrm{H}$. Dibble et $\mathrm{S}$. McPherron vers la base de la séquence, et celui correspondant à la couche $\mathrm{J} 1 / 5 \mathrm{~A}$ vers le milieu.

Pour le reste, nous avons dû concentrer notre attention sur les seuls documents disponibles: le matériel archéologique (état de conservation, caractéristiques technologiques et typologiques) et les coordonnées cartésiennes des objets enregistrées dans les carnets de fouille. Nous avons privilégié l'outil d'analyse des diagrammes de projection.

\section{DONNÉES ARGHÉOLOGIQUES}

En un premier temps, nous avons examiné la répartition stratigraphique des objets roulés, et mis en relation les couches F2 et F3 de F. Bordes avec la couche 3A de H. Dibble et S. McPherron et J2/6B et le sommet de la couche $\mathrm{X} / 7$ (fig. 15).

Nous avons ensuite comparé les principaux niveaux archéologiques des deux fouilles, ce qui a permis de mettre 


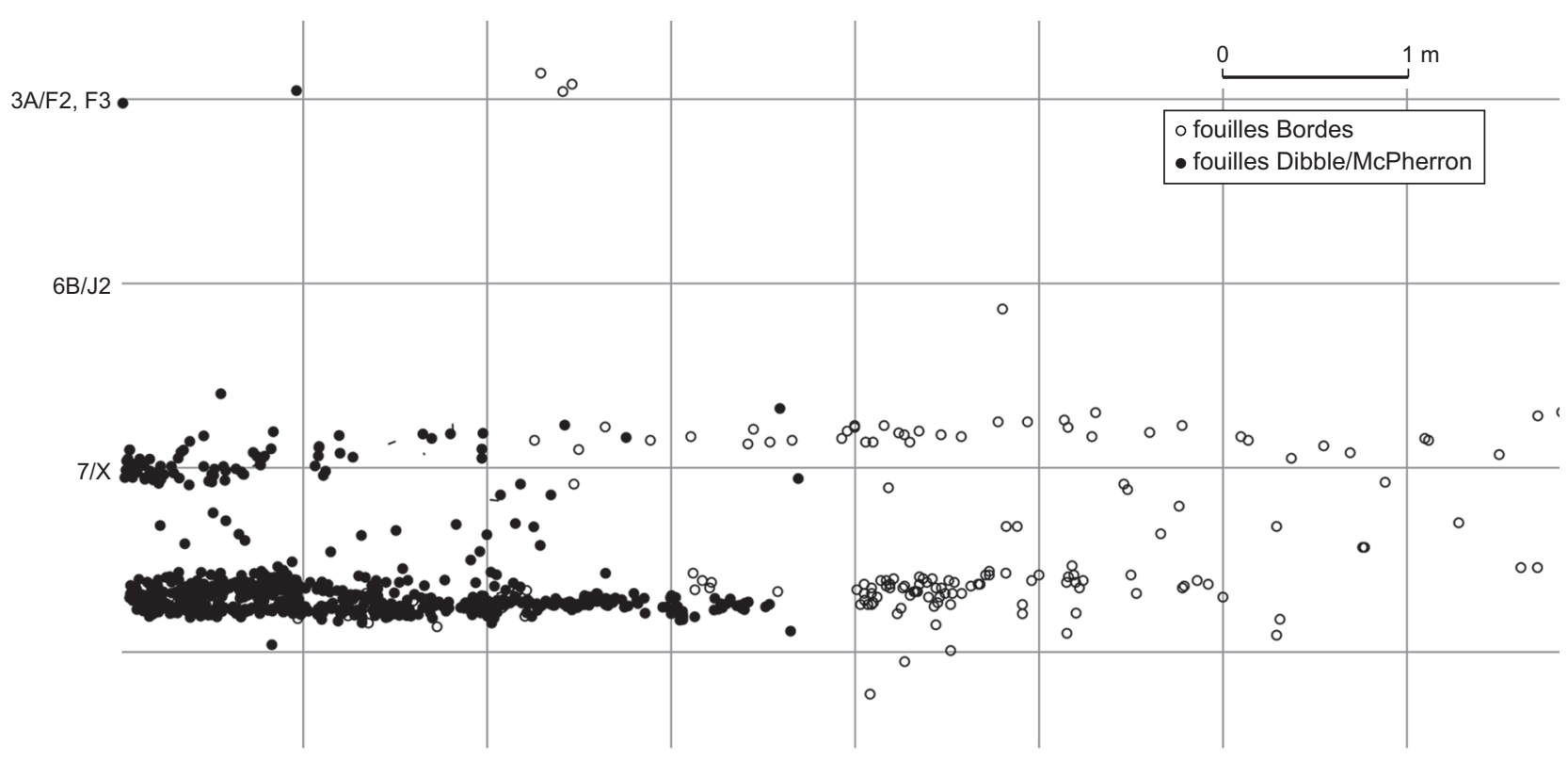

Fig. 15 - Diagramme de projection des objets roulés des deux fouilles (DAO: H. Dibble, Université de Pennsylvanie).

Tabl. V - Tableau synthétique de corrélations des deux séquences stratigraphiques.

\begin{tabular}{|c|c|c|c|}
\hline $\begin{array}{c}\text { Stratigraphie } \\
\text { Bordes }\end{array}$ & \begin{tabular}{|c|} 
Stratigraphie \\
archéologique \\
Dibble, McPherron
\end{tabular} & \begin{tabular}{|c|} 
Niveaux \\
archéologiques \\
Dibble, McPherron
\end{tabular} & $\begin{array}{l}\text { Lithostratigraphie } \\
\text { Golberg, Dibble, } \\
\text { McPherron }\end{array}$ \\
\hline $\mathrm{A} 2$ & \multirow{5}{*}{$\begin{array}{l}1 \mathrm{~B} \\
1 \mathrm{C} \\
1 \mathrm{D}\end{array}$} & & \multirow{5}{*}{1} \\
\hline B & & & \\
\hline C & & & \\
\hline $\mathrm{D}$ & & & \\
\hline $\mathrm{E}$ & & & \\
\hline F1 & 2 & & 2 \\
\hline F2 & \multirow{2}{*}{$3 \mathrm{~A}$} & & \multirow{2}{*}{$3 \mathrm{~A}$} \\
\hline F3 & & & \\
\hline F4 & $3 B$ & & $3 A$ base $+3 B$ \\
\hline G & \multirow{2}{*}{$4 \mathrm{~A}$} & 4A1 & \multirow{2}{*}{$4 \mathrm{~A}$} \\
\hline $\mathrm{H} 1 / \mathrm{H} 2$ & & $4 \mathrm{~A} 2$ & \\
\hline \multirow[t]{2}{*}{11} & 4B & & sommet 4B \\
\hline & \multicolumn{3}{|c|}{ effondrement } \\
\hline 12 & $4 \mathrm{C}$ & $4 \mathrm{C} 1$ et $4 \mathrm{C} 2$ & $4 B$ base $+4 C$ \\
\hline $\mathrm{J} 1$ & $5 \mathrm{~A}$ & $5 \mathrm{~A} 1$ & $5 \mathrm{~A}$ \\
\hline $\mathrm{J} 2$ & $5 B$ & & $5 B$ \\
\hline J3A & \multirow{2}{*}{$6 \mathrm{~A}$} & 6A1 & \multirow{2}{*}{$6 \mathrm{~A}$} \\
\hline J3B & & & \\
\hline \multirow[t]{2}{*}{$\mathrm{J} 3 \mathrm{C}$} & $6 B$ & & $6 \mathrm{~A}+$ sommet $6 \mathrm{~B}$ \\
\hline & \multicolumn{3}{|c|}{ effondrement } \\
\hline X sommet & 7 & & $6 \mathrm{~B}$ base +7 \\
\hline \multicolumn{4}{|l|}{$X$ base } \\
\hline $\mathrm{Y}$ & \multirow{2}{*}{8} & & \multirow{2}{*}{8} \\
\hline Z & & & \\
\hline
\end{tabular}

corrélation à partir des couches à silex roulés corrélation à partir des principales couches archéologiques en parallèle les couches F4 et 3B, I1-I2 et 4B-4C, J3a-J3c et $6 \mathrm{~A}-6 \mathrm{~B}, \mathrm{X}$ base-Y-Z et 8 . La corrélation des couches XYZ et de la couche 8 est également confirmée par le pourcentage très élevé d'objets brûlés, plus de $20 \%$ de l'industrie lithique de ces niveaux.

Dans ce cadre bien établi (tabl. V), nos travaux de terrain et l'analyse du matériel des séries F. bordes permettent d'apporter des nuances sur certains rapprochements ou certaines subdivisons, voire de discuter des attributions culturelles.

Il semblerait que nous n'ayons pas reconnu certaines subdivisions faites par F. Bordes. Toutefois, la quasi-totalité de ses subdivisions sont des distinctions arbitraires et ne représentent pas des changements observables dans les sédiments. Ceci est confirmé par le fait que les subdivisions, regardées sur la coupe, montrent des limites strictement horizontales et plates.

Les projections verticales indiquent que, d'une part, les subdivisions de la couche J proposées par F. Bordes sont arbitraires et d'autre part, la distinction entre I1 et I2 n'est pas très claire, I1 n'étant probablement qu'une simple dilatation vers le haut moins riche qu'en I2 (fig. 16).

Un réexamen du matériel lithique de la couche $\mathrm{G}$ nous amène à proposer une interprétation différente du précédent fouilleur. Si sur les bases de la typologie F. Bordes faisait un parallèle entre ces objets et ceux de la couche F4, nos recherches indiquent qu'elle est plus proche des couches I. 

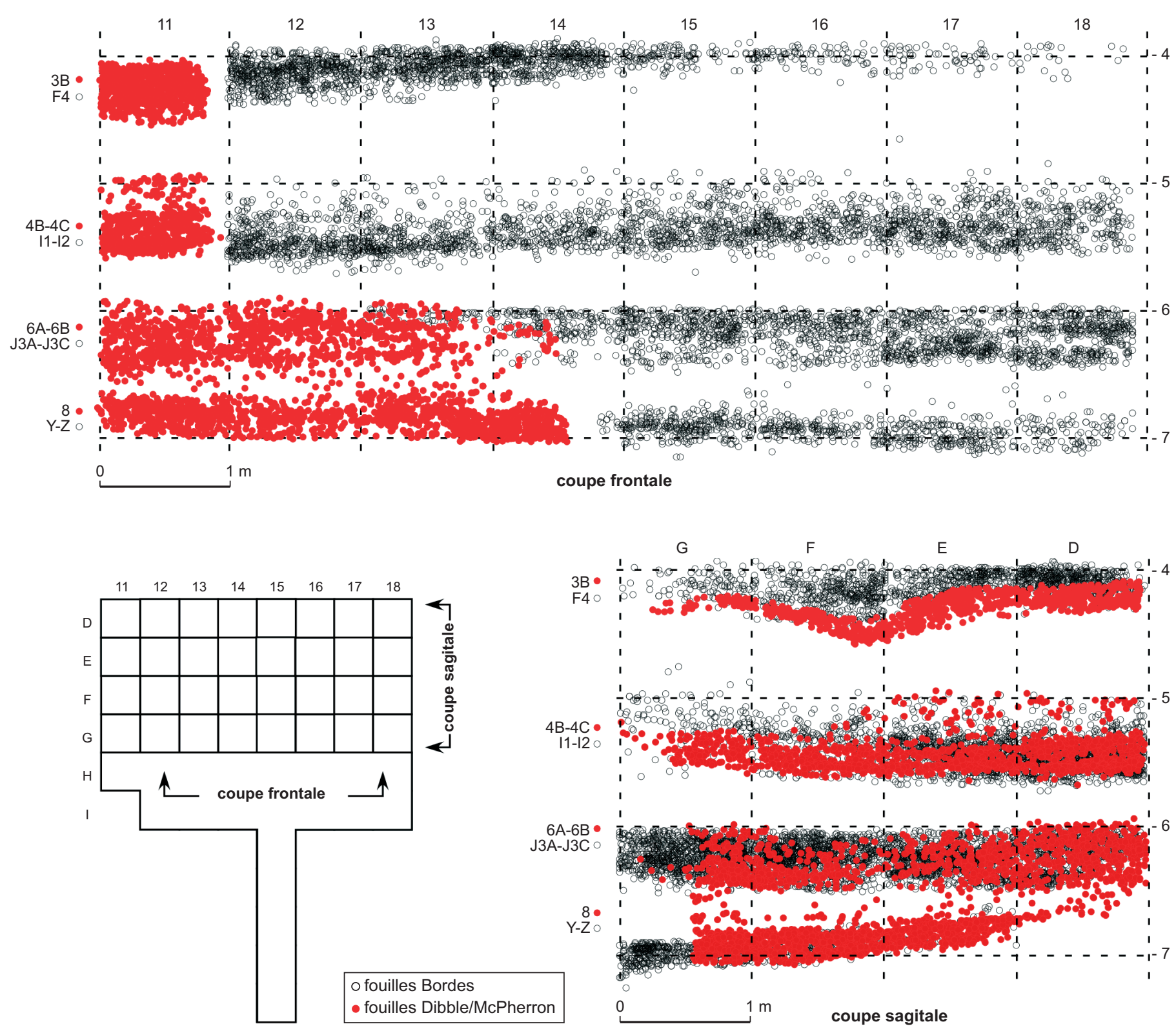

Fig. 16 - Diagramme de projections verticales des objets des principales couches archéologiques (DAO: H. Dibble, Université de Pennsylvanie).

Les couches $\mathrm{F}$, divisées à nouveau arbitrairement en quatre sous-niveaux, constituent le dernier niveau moustérien du Pech de l'Azé IV (McPherron et al., 2005). L'industrie, en particulier en F4, est la plus riche du gisement. Sur la base de la présence de quelques bifaces, F. Bordes attribua ces assemblages au Moustérien de tradition acheuléenne avec une évolution interne du MTA A (niveau F4) au MTA B (niveaux F2 et F1). Après une étude détaillée de la coupe, il est très probable que la stratigraphie de ces niveaux supérieurs soit en réalité bien plus complexe que la description qu'en avait donnée F. Bordes. Nous avons en effet observé et séparé stratigraphiquement un grand nombre de chenaux latéraux d'écoulement près de la coupe la plus au sud des dépôts ${ }^{4}$ dans les bandes $\mathrm{G}$ et $\mathrm{H}$.

4. Il n'est pas sûr que F. Bordes ait parfaitement identifié et séparé ces chenaux. 
Tabl. VI - Inventaire du matériel issu de nos fouilles.

\begin{tabular}{|c|r|c|c|c|r|r|}
\hline Couche & Silex & Galet & Manuport & Minéraux & Os & Total \\
\hline $\mathbf{1}$ & 348 & 0 & 0 & 0 & 224 & 572 \\
\hline $\mathbf{2}$ & 231 & 0 & 0 & 0 & 181 & 412 \\
\hline 3A & 1638 & 0 & 4 & 62 & 746 & 2450 \\
\hline 3B & 2938 & 2 & 0 & 0 & 956 & 3896 \\
\hline 4A & 240 & 3 & 1 & 0 & 811 & 1055 \\
\hline 4B & 49 & 3 & 1 & 10 & 412 & 475 \\
\hline 4C & 650 & 3 & 1 & 20 & 3828 & 4502 \\
\hline 5A & 1452 & 3 & 0 & 39 & 2637 & 4131 \\
\hline 5B & 718 & 144 & 1 & 60 & 1033 & 1956 \\
\hline 6A & 2497 & 8 & 3 & 84 & 4917 & 7509 \\
\hline 6B & 2490 & 1 & 1 & 123 & 5342 & 7957 \\
\hline $\mathbf{7}$ & 4207 & 3 & 0 & 113 & 340 & 4663 \\
\hline $\mathbf{8}$ & 2590 & 0 & 1 & 34 & 1731 & 4356 \\
\hline Total & $\mathbf{2 0 ~ 0 4 8}$ & $\mathbf{1 7 0}$ & $\mathbf{1 3}$ & $\mathbf{5 4 5}$ & $\mathbf{2 3 1 5 8}$ & $\mathbf{4 3 9 3 4}$ \\
\hline
\end{tabular}

Tabl. VII - Correspondance des ensembles archéologiques issus des diverses fouilles.

\begin{tabular}{|c|c|c|}
\hline Bordes & Dibble/McPherron & Couche globale \\
\hline F1, F3 & $3 A$ & I-A \\
\hline F4 & $3 B$ & I-B \\
\hline $\mathrm{G}, \mathrm{H} 1-\mathrm{H} 2$ & $4 \mathrm{~A}$ & II-A \\
\hline $\mathrm{G}, \mathrm{H} 1-\mathrm{H} 2$ & $4 \mathrm{~A}$ & II-A \\
\hline $\mathrm{G}, \mathrm{H} 1-\mathrm{H} 2$ & $4 \mathrm{~A}$ & II-A \\
\hline $\mathrm{G}, \mathrm{H} 1-\mathrm{H} 2$ & $4 \mathrm{~A}$ & II-A \\
\hline $\mathrm{H}$ & $4 \mathrm{~B}$ & II-B \\
\hline 11 & & II-C \\
\hline 11 & $4 C$ & $\mathrm{II}-\mathrm{C}$ \\
\hline 11 & & II-C \\
\hline 12 & $5 \mathrm{~A}$ & III-A \\
\hline $\mathrm{J} 2, \mathrm{~J} 3$ & $5 \mathrm{~B}$ & III-B \\
\hline J3A, J3B & $6 \mathrm{~A}$ & IV-A \\
\hline J3C & $6 B$ & IV-B \\
\hline $\mathrm{x}$ & pas de correspondance & \\
\hline pas de correspondance & 7 & \\
\hline $\mathrm{Y}-\mathrm{Z}$ & 8 & $\mathrm{~V}$ \\
\hline
\end{tabular}

Pour les couches I1 et I2, les analyses ultérieures suggèrent que certaines lentilles de cet ensemble représentent des occupations moustériennes de type Quina (Turq et al., $\left.2008^{5}\right)$.

La méthode d'enregistrement des données, plus précise que celle utilisée par F. Bordes, nous a permis d'identifier des niveaux archéologiques qui avaient pu lui échapper comme ceux individualisés dans les couches $4 \mathrm{~A}$ et $4 \mathrm{C}$. La

5. Depuis la rédaction de cet article, A. Turq a revu une grande partie du matériel issu des fouilles F. Bordes: dans les couches $\mathrm{H}$ et I, et seulement dans celles-ci, il a pu noter la présence de matériel archéologique (débitage et outillage). couche 7, caractérisée par l'aspect roulé du matériel, n'avait pas été individualisée par notre prédécesseur (McPherron et al., 2005).

La séquence des dépôts du Pech de l'Azé IV comprend huit grandes unités stratigraphiques, cinq d'entre elles étant composées au minimum de deux sous-unités. Les couches 1 et 2 sont des dépôts holocènes, dont une grande partie sont des sédiments pléistocènes remaniés provenant du haut de la pente du site, et contiennent peu de matériel archéologique. Les couches 3 à 8 sont pléistocènes avec du Paléolithique moyen. Elles sont très riches en matériel lithique et en faune et représentent une base de données importante sur le comportement et l'adaptation des Néandertaliens. Au cours des dernières fouilles, plus de 20000 objets lithiques et à peu près le même nombre de restes fauniques ont été retrouvés (tabl. VI).

Avant de présenter les industries, l'une de nos préoccupations principales est de voir si les séries issues de nos fouilles correspondent bien à celles de F. Bordes et si nous n'avons pas créé des assemblages fondamentalement différents. En s'appuyant sur les corrélations proposées, il nous est paru important d'effectuer des comparaisons entre les deux séries (tabl. VII). Ces comparaisons sont effectuées par unité stratigraphique correspondante, en tenant compte des différences entre elles ${ }^{6}$.

Bien entendu, il est possible que l'assemblage des fouilles récentes, concentrées sur la partie ouest du site, soit légèrement différent de celui produit par F. Bordes en raison de la variation spatiale sur le site.

Il existe deux tests pertinents pour évaluer la configuration finale de la provenance exacte des sous-collections de F. Bordes: la comparaison de la composition de la collection en termes de grandes catégories lithiques (fig. 17) et la comparaison de la taille des objets (fig. 18). Ces évaluations montrent que les assemblages sont très proches. De même, la taille moyenne des artefacts, séparés par catégories, montre très peu de différences significatives (tabl. VIII). Les artefacts des fouilles de F. Bordes ont tendance à être parfois un peu plus grands que ceux des fouilles récentes. En outre, nous ne savons pas dans quelle mesure cela est dû à la variabilité latérale inhérente au site.

Les objets des deux fouilles ont ensuite été séparés en quatre groupes de taille, puis pesés. La correspondance entre les pourcentages relatifs des fractions de poids est, encore une fois, très bonne (fig. 19).

6. Dans les figures 17, 18 et 19, les appellations des couches correspondent à ce qui est appelé couche globale dans le tableau VII. 

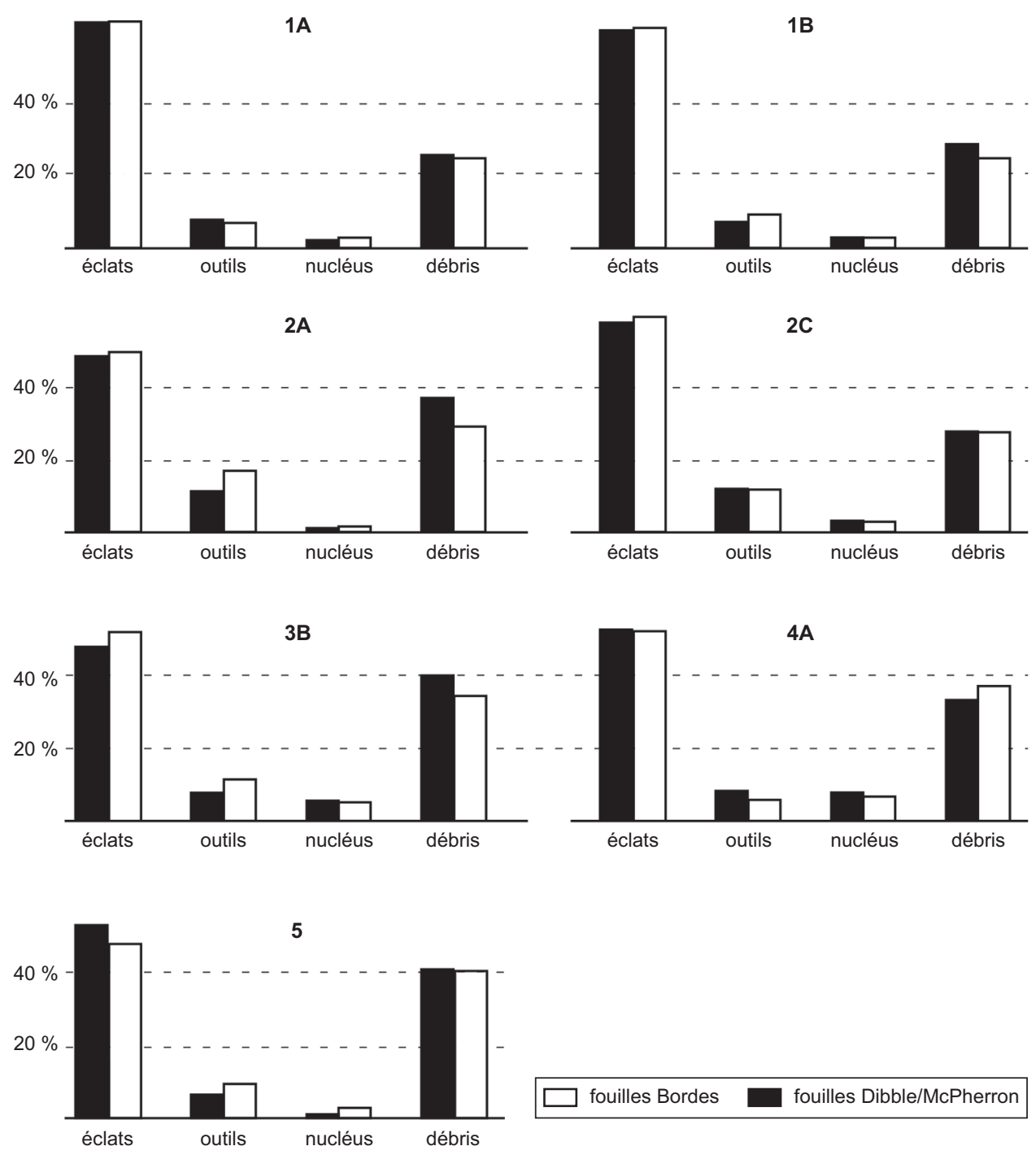

Fig. 17 - Comparaison de la composition globale des ensembles lithiques issus des fouilles de F. Bordes et de H. Dibble et S. McPherron (DAO: H. Dibble, Université de Pennsylvanie).

\section{CHRONOLOGIE}

L'un des buts du projet du site du Pech de l'Azé IV était d'obtenir des datations absolues ${ }^{7}$. Les sites du Pech de l'Azé I et II ont déjà été datés (voir supra, p. 3) et par conséquent, les trois sites peuvent être reliés dans un même cadre chronologique. Les matériaux datés, tels que silex brûlés, os, charbons et dents, sont en nombre relativement important à travers la séquence stratigraphique, excepté pour le niveau $6 \mathrm{~A}$ où les silex brûlés sont moins fréquents. Les

7. Ceci afin de compléter les données existantes (Bowman et al., 1982). travaux AMS et TL sont encore en cours. Nous rapportons ici les résultats de trois dates ESR de la base du niveau 3B.

Trois échantillons ont été sélectionnés pour dater cette couche: E11-846 et E11-999 pour le niveau 3A et E11-1447 pour le niveau 3B. L'échantillon E11-846 a par la suite été divisé en deux: E11-846A et E11-846B, puisqu'ils ne semblaient pas provenir de la même dent. Cela peut se produire puisque les échantillons sont prélevés en bloc avec leur sédiment, et il est possible que deux dents aient été prélevées dans ce bloc, il s'agit alors de deux fragments. L'échantillon E11-999A provient d'une dent complète, les E11-1447A et E11-1447B d'une dent presque complète. Ainsi, les résultats 


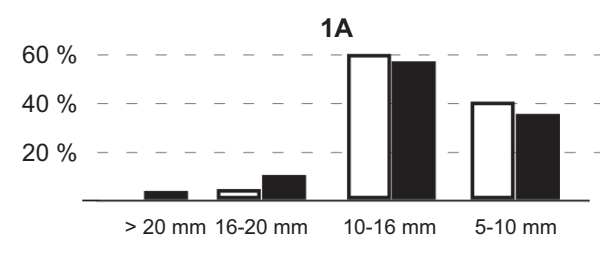

2A

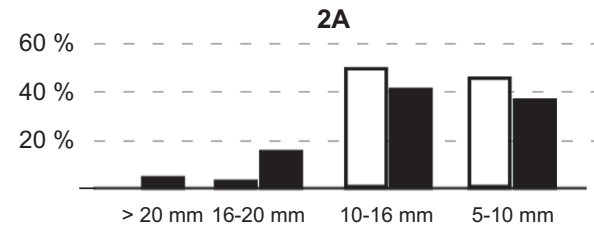

3A

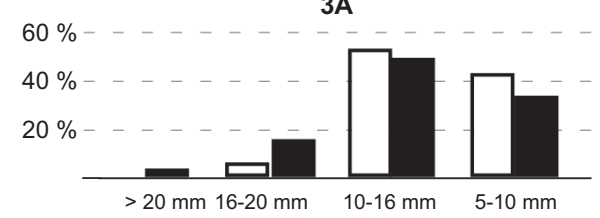

4A

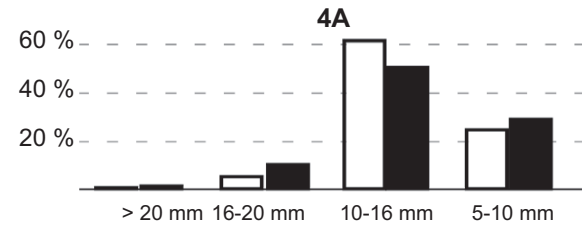

$1 \mathrm{~B}$

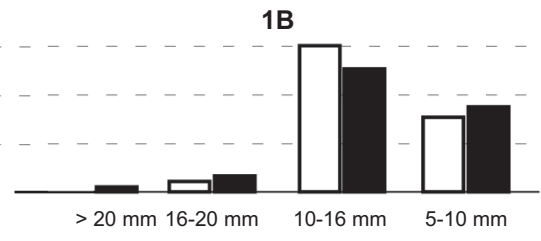

$2 \mathrm{C}$

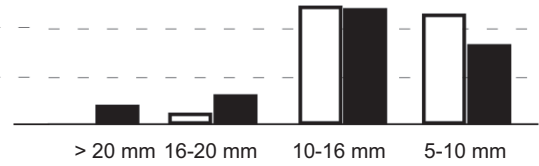

3B

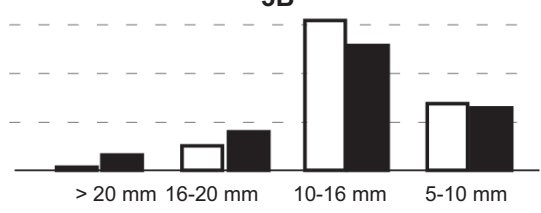

5

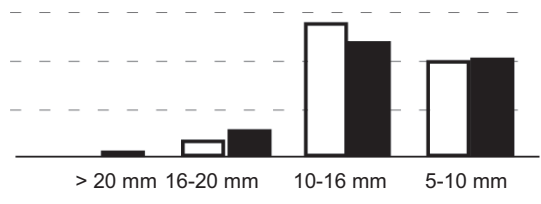

fouilles Dibble/McPherron

Fig. 18 - Comparaison des classes dimensionnelles des ensembles lithiques issus des fouilles de F. Bordes et de H. Dibble et S. McPherron (DAO: H. Dibble, Université de Pennsylvanie).

Tabl. VIII - Comparaison des moyennes des longueurs des outils, des éclats et des nucléus. Les résultats en gras correspondent à une différence significative $(P<.05)$.

\begin{tabular}{|c|c|c|c|c|c|c|}
\hline \multirow{2}{*}{$\begin{array}{l}\text { Couche } \\
\text { globale }\end{array}$} & \multicolumn{2}{|c|}{ Outils } & \multicolumn{2}{|c|}{ Éclats } & \multicolumn{2}{|c|}{ Nucléus } \\
\hline & $\begin{array}{c}\text { nouvelle } \\
\text { collection }\end{array}$ & \begin{tabular}{|c|} 
collection \\
Bordes
\end{tabular} & $\begin{array}{c}\text { nouvelle } \\
\text { collection }\end{array}$ & $\begin{array}{c}\text { collection } \\
\text { Bordes }\end{array}$ & $\begin{array}{c}\text { nouvelle } \\
\text { collection }\end{array}$ & $\begin{array}{c}\text { collection } \\
\text { Bordes }\end{array}$ \\
\hline I-A & 39,8 & 44,4 & 32,4 & 33,8 & 42,6 & 46,6 \\
\hline I-B & 37,9 & 43,1 & 32,7 & 35,1 & 45,8 & 49,4 \\
\hline II-A & 49,7 & 50,9 & 34,6 & 35,8 & 47,6 & 55,0 \\
\hline II-B & 52,2 & 53,8 & 36,6 & 38,8 & 84,5 & 53,5 \\
\hline II-C & 54,8 & 58,8 & 33,8 & 40,3 & 52,0 & 52,3 \\
\hline III-A & 56,5 & 55,7 & 36,4 & 43,1 & 49,8 & 51,7 \\
\hline III-B & 50,8 & 51,8 & 37,0 & 39,2 & 47,8 & 44,9 \\
\hline IV-A & 50,0 & 41,4 & 38,5 & 36,4 & 40,7 & 39,7 \\
\hline V & 47,7 & 48,7 & 35,4 & 37,4 & 41,9 & 47,2 \\
\hline
\end{tabular}

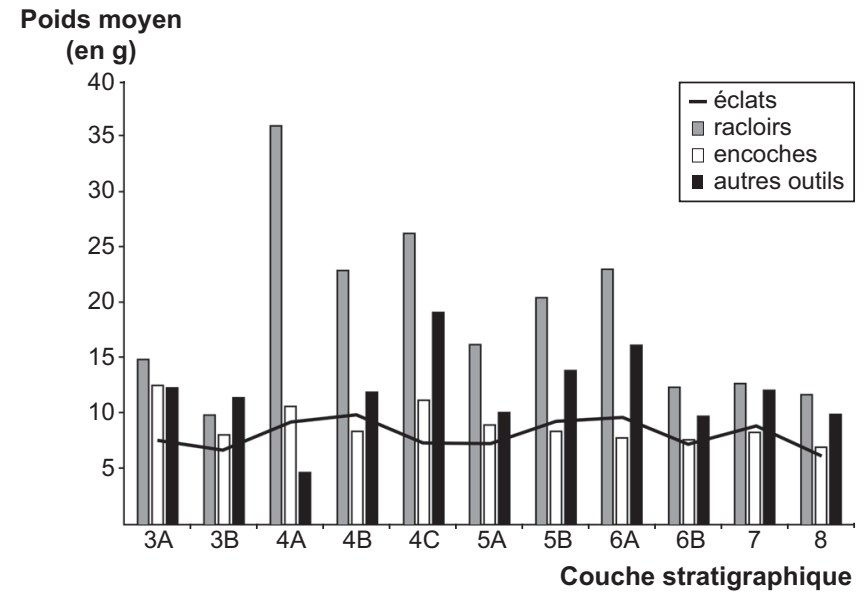

Fig. 19 - Moyenne du poids des grands groupes typologiques par couche (DAO: H. Dibble, Université de Pennsylvanie). 
Tabl. IX - Situation et dates par résonance paramagnétique électronique (RPE) pour les fragments d'émail de dent au Pech de l'Azé IV avec un taux d'humidité actuel de $7 \%$ et en prenant en compte une erreur de $10 \%$.

\begin{tabular}{|c|c|c|c|}
\cline { 2 - 4 } \multicolumn{1}{c|}{} & Couche & EU âge (ka) & LU âge (ka) \\
\hline E11-846A & $3 \mathrm{~A}$ & $56 \pm 7$ & $60 \pm 8$ \\
\hline $\mathrm{E} 11-846 \mathrm{~B}$ & $3 \mathrm{~A}$ & $50 \pm 7$ & $51 \pm 7$ \\
\hline $\mathrm{E} 11-999 \mathrm{~A}$ & $3 \mathrm{~A}$ & $47 \pm 6$ & $48 \pm 7$ \\
\hline $\mathrm{E} 11-1447 \mathrm{~A}$ & $3 \mathrm{~B}$ & $48 \pm 8$ & $49 \pm 8$ \\
\hline $\mathrm{E} 11-1447 \mathrm{~B}$ & $3 \mathrm{~B}$ & $50 \pm 6$ & $52 \pm 7$ \\
\hline $\begin{array}{c}\text { Moyenne arithmétique } \\
(\mathbf{n}=\mathbf{4}) \text { sans 846A }\end{array}$ & & $\mathbf{4 9} \pm \mathbf{2}$ & $\mathbf{5 0 \pm \mathbf { 2 }}$ \\
\hline $\begin{array}{c}\text { Moyenne arithmétique } \\
(\mathbf{n}=\mathbf{5})\end{array}$ & & $\mathbf{5 0 \pm 3}$ & $\mathbf{5 2} \pm \mathbf{5}$ \\
\hline
\end{tabular}

Tabl. $\mathbf{X}$ - Situation et dates RPE pour les fragments d'émail de dent au Pech de l'Azé IV avec l'hypothèse d'un taux d'humidité élevé à $20 \%$.

\begin{tabular}{|c|c|c|c|}
\cline { 2 - 4 } \multicolumn{1}{c|}{} & Couche & EU âge (ka) & LU âge (ka) \\
\hline $\mathrm{E} 11-846 \mathrm{~A}$ & $3 \mathrm{~A}$ & $62 \pm 8$ & $67 \pm 9$ \\
\hline $\mathrm{E} 11-846 \mathrm{~B}$ & $3 \mathrm{~A}$ & $56 \pm 8$ & $57 \pm 8$ \\
\hline $\mathrm{E} 11-999 \mathrm{~A}$ & $3 \mathrm{~A}$ & $53 \pm 7$ & $54 \pm 7$ \\
\hline $\mathrm{E} 11-1447 \mathrm{~A}$ & $3 \mathrm{~B}$ & $55 \pm 9$ & $55 \pm 9$ \\
\hline $\mathrm{E} 11-1447 \mathrm{~B}$ & $3 \mathrm{~B}$ & $56 \pm 7$ & $59 \pm 8$ \\
\hline $\begin{array}{c}\text { Moyenne arithmétique } \\
(\mathbf{n}=\mathbf{4} \text { ) sans 846A }\end{array}$ & & $\mathbf{5 5} \pm \mathbf{1}$ & $\mathbf{5 6} \pm \mathbf{2}$ \\
\hline $\begin{array}{c}\text { Moyenne arithmétique } \\
(\mathbf{n = 5})\end{array}$ & & $\mathbf{5 6} \pm \mathbf{3}$ & $\mathbf{5 8} \pm \mathbf{5}$ \\
\hline
\end{tabular}

représentent probablement les âges de quatre dents différentes (tabl. IX et X).

L'échantillon E11-846A avait la plus grande quantité d'absorption en uranium pendant son enfouissement (6,6 ppm dans la dentine), alors que les autres avaient de plus petites valeurs variant de $0,9 \mathrm{ppm}$ à environ $3,2 \mathrm{ppm}$. Ce taux élevé a mené à l'extension des estimations d'âge EU (Early Uptake) et LU (Linear Uptake), alors que dans les autres dents il n'y a pratiquement aucune différence. Aucun des fragments d'émail n'a eu de quantité significative d'uranium incorporé.

Seul E11-846A montre un écart significatif par rapport à la tendance des quatre autres âges des échantillons, mais son âge dépend sans aucun doute du véritable modèle d'Uptake. Pour l'instant, nous avons laissé le résultat au niveau du calcul des âges moyens, puisqu'il n'a presque aucun effet de toute façon (dans l'incertitude).

En utilisant le contenu d'humidité actuelle, les âges moyens (et $\pm 1 \sigma$ de la moyenne arithmétique) sont $50 \pm 3 \mathrm{ka}$ pour l'hypothèse EU et $52 \pm 5$ ka pour l'hypothèse LU, provoquant une fourchette de l'âge entre $47 \mathrm{ka}$ à $57 \mathrm{ka}$ en utilisant $\mathrm{n}=5$ comme moyenne. Si nous excluons l'extérieur, l'âge EU est de $49 \pm 2$ ka et l'âge LU de $50 \pm 2$ ka, nous donnant une fourchette de 47-52 ka. Les âges calculés sont tout à fait sensibles à la teneur en humidité des sédiments qui sont employés dans le calcul parce qu'il y a une très petite dose associée à l'uranium dans la dent. Si nous faisons l'hypothèse d'une teneur moyenne beaucoup plus élevée en humidité de $20 \%$ et la laissant varier entre $10 \%$ et $30 \%$ (en utilisant $20 \pm 10 \%$ dans le calcul), les dates augmentent. Dans ces conditions hypothétiques, les âges moyens EU et LU sont respectivement de $55 \pm 1$ ka et $56 \pm 2 \mathrm{ka}$, nous donnant une fourchette de dates de 54-58 ka, en incluant toutes les données obtenues, nous avons des dates moyennes EU et LU respectivement de $56 \pm 2$ ka et $58 \pm 3 \mathrm{ka}$, pour une fourchette d'âge de $54 \mathrm{ka}$ à $60 \mathrm{ka}$. Le meilleur choix pour la teneur en humidité est considéré comme étant l'évaluation de l'humidité actuelle car, de toute évidence, les conditions durant la dernière glaciation étaient plus sèches qu'aujourd'hui. Les valeurs de l'âge moyen avec $n=5$ constituent le meilleur choix, plutôt que les valeurs avec $n=4$. Effectivement, l'annexe ne peut pas être statistiquement exclue en se basant sur le fait que sa valeur se situe autour de $\pm 2 \sigma$ de la moyenne arithmétique tout en comprenant des incertitudes. Par contre, il n'y en a pratiquement aucune liée à la prise en uranium dans les quatre dents inférieures, ainsi la datation U-série n'est pas nécessaire pour l'affiner. Les quatre dates inférieures sont bien groupées et semblent suggérer clairement que E11-846A ne peut pas être aussi vieux que les âges types EU et LU le suggèrent.

Les datations sont basées sur une teneur en humidité de $7 \%$ (la moyenne de huit échantillons prélevés de la coupe du carré E11 = 7,03 $\pm 2,97 \%$ ). Une incertitude dans la teneur en humidité de sédiment de $\pm 10 \%$ a été employée pour le calcul de la dose bêta du sédiment, alors qu'on assumait que le taux de la dose gamma plus cosmique (déterminé à l'aide de six dosimètres de thermoluminescence [TLD] implantés à proximité) possède une erreur totale de $\pm 15 \%$. La couverture fournie par les TLD était excellente pour les dents mises au jour. Cette erreur est environ deux fois plus grande que la valeur $\pm 1 \sigma$ obtenue comme la variance de la valeur des six dosimètres mesurés. L'erreur ajoutée est incluse pour traiter des variations locales du non expliqué par les dosimètres et toute variation du contenu en humidité dont la durée de l'enfouissement ne peut être déterminée.

Nous avons eu depuis une nouvelle datation concernant la couche 8 qui est en cours de publication: 99,9 $\pm 5,4 \mathrm{ka}$ (Dibble et al., 2009). À ce stade et dans l'attente des résultats d'analyse des autres échantillons et des données de 
Tabl. XI - Synthèse des données chronologiques.

\begin{tabular}{|c|c|c|c|}
\hline $\begin{array}{c}\text { Stratigraphie } \\
\text { Dibble/McPherron }\end{array}$ & Datations & Données paléontologiques & \begin{tabular}{|c|} 
Proposition \\
Bordes
\end{tabular} \\
\hline 2 & & & Würm II \\
\hline \multicolumn{4}{|c|}{ lacune stratigraphique et phase d'érosion } \\
\hline $3 \mathrm{~A}$ & 54 à $60 \mathrm{Ka}$ & bovidés dominant & Würm II \\
\hline $3 B$ & & renne dominant & Würm II \\
\hline \multicolumn{4}{|c|}{ lacune stratigraphique et phase d'érosion } \\
\hline $4 \mathrm{~A}$ & & renne dominant & Würm I \\
\hline $4 \mathrm{~B}$ & & renne dominant & Würm I \\
\hline $4 \mathrm{C}$ & & renne dominant & Würm I \\
\hline $5 \mathrm{~A}$ & & renne & Würm I \\
\hline 5B & & cerf dominant, apparition du renne & Würm I \\
\hline \multicolumn{4}{|c|}{ lacune stratigraphique } \\
\hline $6 \mathrm{~A}$ & & cerf dominant, chevreuil à son maximum & Würm I \\
\hline $6 B$ & & cerf dominant, chevreuil & Würm I \\
\hline 7 & & cerf dominant, chevreuil, cheval & Würm I \\
\hline 8 & $99,9 \pm 5,4$ & cerf dominant, chevreuil, cheval & Würm I \\
\hline
\end{tabular}

paléontologie, nous avons souhaité synthétiser les données (tabl. XI).

Les quelques éléments de datation semblent confirmer les premières approches faites par F. Bordes. Si l'on peut attribuer l'ensemble de base (couches 8 à $6 \mathrm{~A}$ ) à une phase du stade isotopique 5 et le sommet de la séquence (au moins la couche 3A) au début du stade isotopique 3, nous ne sommes pas en mesure de replacer pour l'heure l'ensemble médian (couche 4A à 5B).

\section{LES INDUSTRIES LITHIQUES}

Avant même de parler des industries lithiques, il nous paraît nécessaire de présenter le potentiel des ressources en matières premières lithiques. De plus, si l'ensemble du matériel a été étudié, nous souhaitons avant la publication finale poursuivre le travail sur les séries en essayant notamment des remontages pour, d'une part, valider l'intégrité des couches archéologiques observées à la fouille et d'autre part, acquérir des informations complémentaires aux premières analyses technologiques. Par ailleurs, l'approche techno-économique dans laquelle nous voulons nous engager, qui doit prendre en compte les données régionales, ne pourra être véritablement lancée que lorsque nous disposerons des résultats concernant la faune et les datations. Pour ces raisons, notre présentation des industries se réduit à une approche très globale s'appuyant essentiellement sur la méthode F. Bordes et est accompagnée d'une abondante illustration au trait.

\section{LES MATIÈRES PREMIÈRES LITHIQUES}

Toute étude de l'origine des matières premières lithiques commence par l'estimation des ressources du secteur géographique du site et donc par une approche géologique (Demars, 1982; Morala, 1983; Geneste, 1985; SéronieVivien, Séronie-Vivien, 1987; Turq, 2000). Le Sarladais correspond à un synclinorium orienté sud-est/nord-ouest, occupé par des formations du Crétacé supérieur (Turonien, Coniacien, Santonien et base de Campanien) (fig. 20). Au sein de cette structure, on observe plusieurs rides anticlinales dont celle du Pech de l'Azé (Turq et al., 1999) qui a contribué au développement du karst ayant servi de structure d'accueil pour les Paléolithiques et a permis aux niveaux à silex de la base du Coniacien d'affleurer. Cette ride anticlinale, observée uniquement le long du ruisseau de Farge, ne mesure que deux kilomètres.

Dans l'environnement proche, les silex du Coniacien apparaissent dans les calcaires, à quelques mètres des sites du Pech de l'Azé sur les pentes du coteau, de part et d'autre du vallon situé en avant du site mais aussi de façon plus ou moins régulière sur l'ensemble du cour de l'anticlinal. On les retrouve, toujours in situ, vers le nord, le long de la vallée de l'Énéa (à $7 \mathrm{~km}$ vers Saint-Nathalène), au sud/sudest, le long de la vallée de la Dordogne, sur la rive gauche légèrement en amont de Domme $(5 \mathrm{~km})$ et sur la rive droite à Vitrac $(4 \mathrm{~km})$. En position secondaire, ils sont présents dans les altérites à quelques centaines de mètres à l'ouest du Pech de l'Azé, mais surtout dans les alluvions du ruisseau 


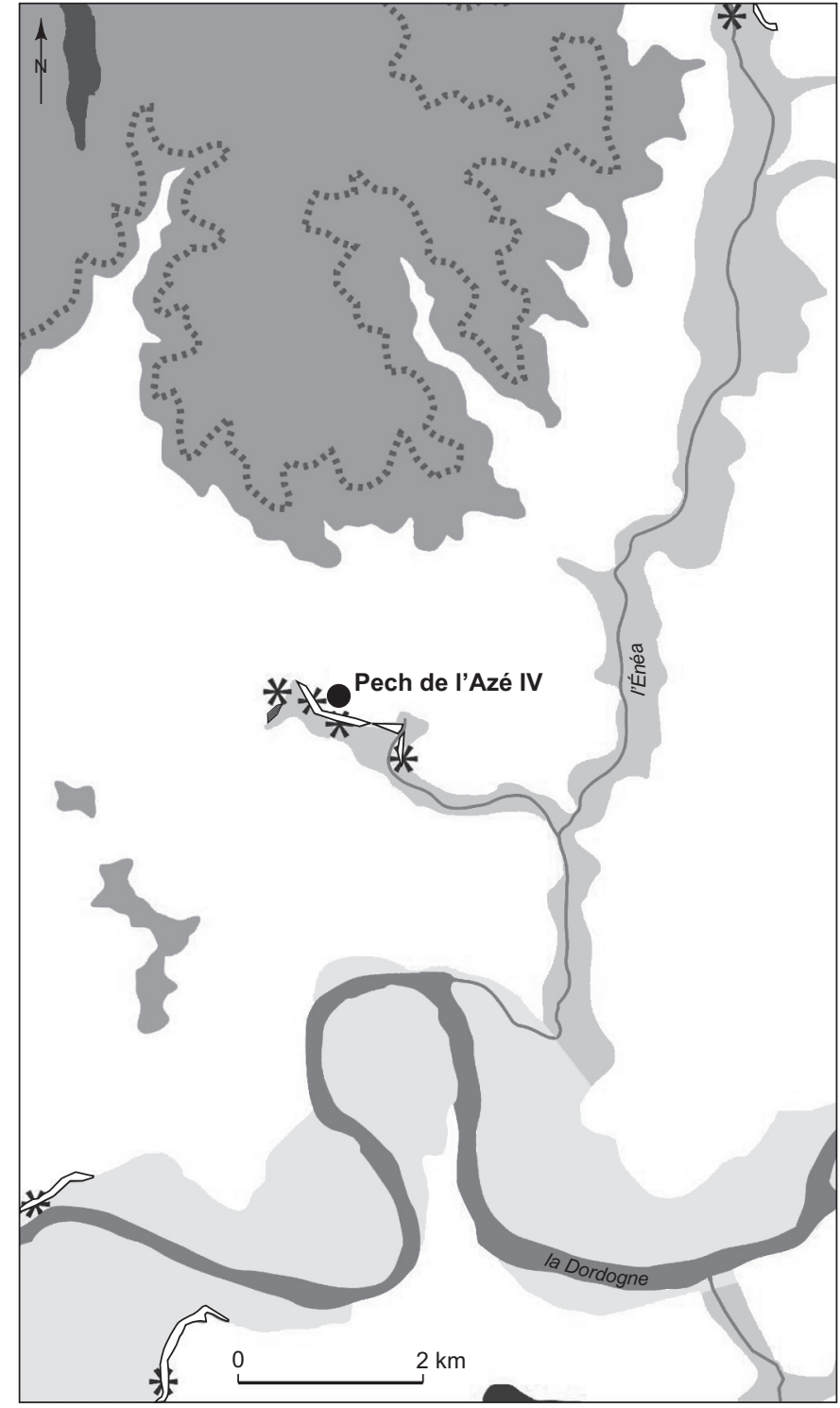

* gîte de silex in situ
affleurement des bancs de silex de la base du Coniacien
affleurement des bancs de silex de la base du Santonien
formations alluviales de l'Énéa (silex blond, gris-noir du Sénonien et calcédoine)
formations alluviales de la Dordogne (silex blond, gris-noir du Sénonien, jaspes,
silex jurassiques, silex tertiaires, quartz, etc.)
formation tertiaire lacustre (silex calcédonieux)
affleurement du Campanien (silex blond et gris-noir du Sénonien)
affleurement du Santonien (silex blond, gris-noir du Sénonien et calcédoine)
affleurement du Coniacien (silex blond, gris-noir du Sénonien et calcédoine)

Fig . 20 - Carte de localisation des principaux affleurements de matières premières lithiques autour du site (DAO: A. Turq, Musée national de Préhistoire).
Tabl. XII - Les matières premières lithiques utilisées.

\begin{tabular}{|c|r|c|c|c|c|c|r|c|}
\hline Couche & $\begin{array}{c}\text { Silex } \\
\text { local }\end{array}$ & $\begin{array}{c}\text { Silex } \\
\text { tertiaire }\end{array}$ & $\begin{array}{c}\text { Autre } \\
\text { silex }\end{array}$ & Jaspe & Quartz & Bergerac & Ind. & $\begin{array}{c}\% \\
\text { exotique }\end{array}$ \\
\hline 3A & 1585 & 24 & 0 & 2 & 13 & 1 & 18 & 2,4 \\
\hline 3B & 2850 & 33 & 1 & 0 & 1 & 0 & 30 & 1,2 \\
\hline 4A & 220 & 17 & 0 & 0 & 0 & 0 & 6 & 7,1 \\
\hline 4B & 51 & 1 & 0 & 0 & 0 & 0 & 0 & 2 \\
\hline 4C & 626 & 14 & 0 & 0 & 0 & 0 & 14 & 2,2 \\
\hline 5A & 1390 & 20 & 0 & 3 & 0 & 0 & 26 & 1,6 \\
\hline 5B & 816 & 14 & 0 & 1 & 9 & 2 & 19 & 3,1 \\
\hline 6A & 2343 & 48 & 1 & 1 & 9 & 2 & 43 & 2,5 \\
\hline 6B & 2383 & 42 & 1 & 4 & 7 & 1 & 57 & 2,3 \\
\hline 8 & 2240 & 13 & 4 & 1 & 0 & 0 & 337 & 0,8 \\
\hline Total & $\mathbf{1 4} 5 \mathbf{5 0 4}$ & $\mathbf{2 2 6}$ & $\mathbf{4}$ & $\mathbf{1 2}$ & $\mathbf{3 9}$ & $\mathbf{4}$ & $\mathbf{5 5 0}$ & $\mathbf{1 , 9}$ \\
\hline
\end{tabular}

de Farges, ceux de l'Énéa et de la Dordogne y compris en amont de son confluent avec ce dernier tributaire.

Moins abondants que les précédents, les silex du Santonien existent à la base de la partie supérieure de cette formation dans un calcaire crayeux blanchâtre peu épais (Capdeville, 1987, p. 14). On les retrouve en position dérivée dans les altérites recouvrant les zones planes ou dans les alluvions de l'Énéa. Le Campanien n’affleure qu'au nord de Sarlat sur les points culminants et peut livrer ponctuellement des silicifications.

Les silex calcédonieux peuvent avoir deux origines: des géodes formées dans les assises crétacées et une meuliérisation de calcaire tertiaire. Les premières existent ponctuellement dans la vallée de l'Énéa, les secondes sont sur la rive gauche de la Dordogne, au massif de la plaine de Bor et sur celui plus en amont de la forêt, dans les altérites et les dépôts de pente. Cette formation, drainée par les systèmes hydrographiques de la Germaine et du Céou, a largement alimenté des silicifications tertiaires aux alluvions de la Dordogne où elles se mélangent à celles issues du Massif central.

Les formations quaternaires sont des gîtes privilégiés particulièrement propices: quantité, qualité et régénérescence après chaque crue (mélange des alluvions et transport ainsi que reprise des éléments arrachés aux berges). Les ressources sont différentes entre la vallée de la Dordogne et celle de l'Énéa. Cette dernière ne transporte que des roches locales (calcaire, silex, grès) issues de leur bassin versant alors que la première charrie, en plus, des roches locales issues de son bassin amont (roches métamorphiques, plutoniques, quartz, quartzites), mais aussi des silicifications: jaspes de l'Hettangien, silex jurassique et tertiaire. 


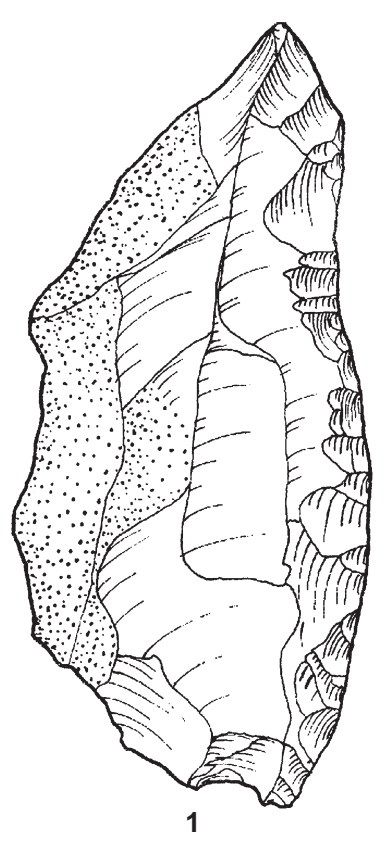

0 $5 \mathrm{~cm}$
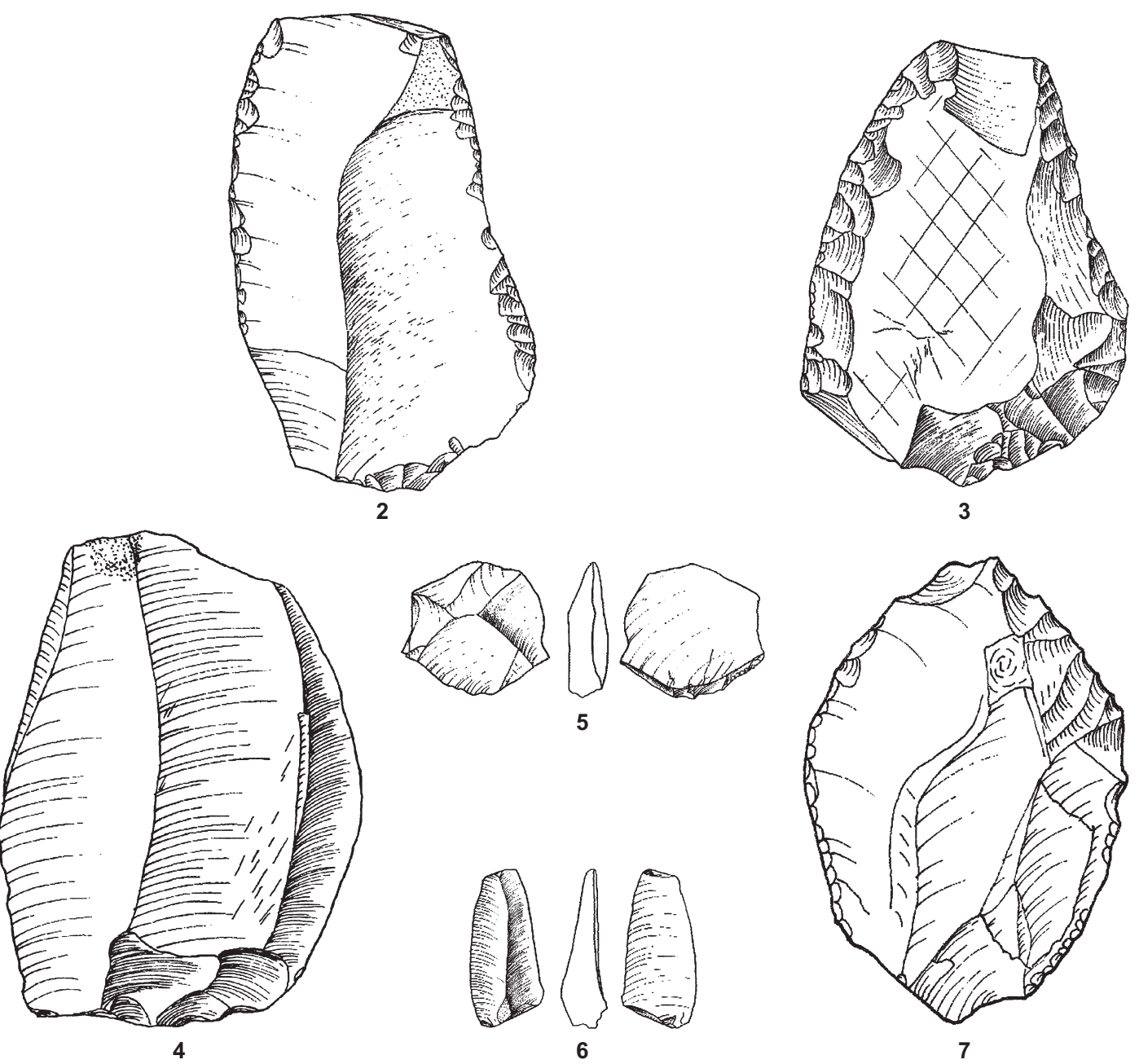

Fig. 21 - Couche 8: 1, racloir simple convexe; 2 et 3, racloir double convexe; 4 et 5, éclat Levallois; 6, lame; 7, racloir double convergent sur support Levallois (dessin: T. Tsenova).

En général, il est typique pour les sites européens du Paléolithique moyen que la majorité de l'assemblage lithique soit composée de matières premières disponibles dans l'environnement immédiat. C'est le cas au Pech de l'Azé IV avec une moyenne de $98 \%$ de l'assemblage en matière première locale (tabl. XII). Cela reste tout à fait cohérent pour toutes les couches, à l'exception du niveau 8 où le matériel local atteint $99,2 \%$. Comme indiqué plus haut, cela semble refléter l'importation sur le site de supports bifaciaux/outils préfabriqués plutôt que de nucléus/nodules de matière première exogène. La matière première exotique la plus commune est la calcédoine, mais le silex de Bergerac, d'une qualité particulièrement supérieure, disponible à plus de $50 \mathrm{~km}$ de distance, représente un très faible pourcentage dans certains assemblages.

\section{LA COUCHE 8}

Comme pour les couches 6 et 5 , la couche 8 a un indice Levallois modérément élevé et un indice de racloir relativement fort (dominé par les racloirs simples, rectilignes et convexes). C'est probablement, comme le pensait déjà F. Bordes pour les niveaux correspondants ( $\mathrm{Y}$ et $\mathrm{X}$ ), un Moustérien typique (fig. 21, tabl. XIII à XIX). Cependant, cette couche a des caractéristiques uniques. D'une part, elle a le plus faible pourcentage en matières premières exotiques avec $0,8 \%$ (la moyenne pour les autres couches est de $1,9 \%$, tabl. XVI). Les objets en matériaux exotiques ont été introduits dans le site sous forme d'éclats ou d'outils. Cela reflète les différentes utilisations du site ou les modes globaux d'occupation du territoire au cours de cette période. 


\begin{tabular}{|c|c|c|c|c|c|c|c|}
\hline Couche & $\begin{array}{c}\text { Indice Levallois } \\
\text { typologique }\end{array}$ & $\begin{array}{l}\text { Indice } \\
\text { racloir }\end{array}$ & $\begin{array}{c}\text { Indice acheuléen } \\
\text { unifacial }\end{array}$ & $\begin{array}{c}\text { Groupe I } \\
\text { Levallois }\end{array}$ & $\begin{array}{c}\text { Groupe II } \\
\text { Moustérien }\end{array}$ & $\begin{array}{c}\text { Groupe III } \\
\text { Paléolithique supérieur }\end{array}$ & $\begin{array}{c}\text { Groupe IV } \\
\text { denticulé }\end{array}$ \\
\hline 3A & 10,27 & 5,32 & 0,02 & 10,27 & 21,29 & 4,94 & 17,11 \\
\hline 3B & 25,42 & 6,92 & 0,00 & 25,42 & 10,84 & 3,74 & 13,46 \\
\hline 4A & 27,45 & 39,22 & 0,00 & 27,45 & 45,10 & 1,96 & 3,92 \\
\hline 4B & 18,18 & 54,55 & 0,00 & 18,18 & 18,18 & 0,00 & 0,00 \\
\hline 4C & 20,63 & 47,62 & 0,00 & 20,63 & 53,17 & 1,59 & 6,35 \\
\hline 5A & 35,88 & 24,05 & 0,01 & 35,88 & 28,63 & 3,05 & 1,15 \\
\hline 5B & 13,88 & 12,65 & 0,00 & 13,88 & 13,47 & 2,86 & 1,63 \\
\hline 6A & 43,30 & 11,41 & 0,00 & 43,30 & 13,59 & 2,17 & 5,07 \\
\hline 6B & 34,48 & 3,63 & 0,00 & 34,48 & 7,99 & 2,18 & 4,90 \\
\hline 7 & 4,18 & 3,36 & 0,00 & 4,18 & 4,59 & 0,98 & 2,68 \\
\hline 8 & 37,73 & 23,18 & 0,00 & 37,73 & 27,27 & 2,27 & 2,27 \\
\hline
\end{tabular}

Tabl. XIII - Principaux indices et groupes typologiques et technologiques (méthode F. Bordes).

Tabl. XIV - Compte des nucléus: $\boldsymbol{a}$, en nombre; $\boldsymbol{b}$, en pourcentage.

\begin{tabular}{|c|c|c|c|c|c|c|r|r|r|}
\hline Couche & Informe & Une surface & Levallois & Discoïde & Kombewa & Biface & Autre & Non étudiable & Total \\
\hline 3A & 8 & 11 & 1 & 0 & 3 & 0 & 11 & 14 & $\mathbf{4 8}$ \\
\hline 3B & 8 & 54 & 1 & 2 & 6 & 0 & 4 & 14 & $\mathbf{8 9}$ \\
\hline 4A & 0 & 3 & 0 & 0 & 0 & 0 & 0 & 1 & $\mathbf{4}$ \\
\hline 4B & 0 & 0 & 0 & 0 & 0 & 0 & 1 & 0 & $\mathbf{1}$ \\
\hline 4C & 2 & 3 & 1 & 0 & 2 & 0 & 4 & 7 & $\mathbf{1 9}$ \\
\hline 5A & 5 & 21 & 0 & 0 & 4 & 0 & 2 & 16 & $\mathbf{4 8}$ \\
\hline 5B & 11 & 14 & 2 & 0 & 4 & 0 & 7 & 5 & $\mathbf{4 3}$ \\
\hline 6A & 10 & 98 & 1 & 1 & 18 & 0 & 12 & 21 & $\mathbf{1 6 1}$ \\
\hline 6B & 28 & 136 & 24 & 3 & 16 & 2 & 28 & 26 & $\mathbf{2 6 3}$ \\
\hline $\mathbf{7}$ & 33 & 99 & 10 & 6 & 29 & 0 & 33 & 43 & $\mathbf{2 5 3}$ \\
\hline $\mathbf{8}$ & 5 & 19 & 2 & 1 & 3 & 0 & 3 & 8 & $\mathbf{4 1}$ \\
\hline Total & $\mathbf{1 1 0}$ & $\mathbf{4 5 8}$ & $\mathbf{4 2}$ & $\mathbf{1 3}$ & $\mathbf{8 5}$ & $\mathbf{2}$ & $\mathbf{1 0 5}$ & $\mathbf{1 5 5}$ & $\mathbf{9 7 0}$ \\
\hline
\end{tabular}

\begin{tabular}{|c|c|c|c|c|c|c|c|c|}
\hline Couche & Informe & Une surface & Levallois & Discoïde & Kombewa & Biface & Autre & Total \\
\hline 3A & 23,5 & 32,4 & 2,9 & 0 & 8,8 & 0,0 & 32,4 & $\mathbf{1 0 0}$ \\
\hline 3B & 10,7 & 72,0 & 1,3 & 2,7 & 8,0 & 0,0 & 5,3 & $\mathbf{1 0 0}$ \\
\hline 4A & 0,0 & 100,0 & 0,0 & 0,0 & 0,0 & 0,0 & 0,0 & $\mathbf{1 0 0}$ \\
\hline 4B & 0,0 & 0,0 & 0,0 & 0,0 & 0,0 & 0,0 & 100,0 & $\mathbf{1 0 0}$ \\
\hline 4C & 16,7 & 25,0 & 8,3 & 0,0 & 16,7 & 0,0 & 33,3 & $\mathbf{1 0 0}$ \\
\hline 5A & 15,6 & 65,6 & 0,0 & 0,0 & 12,5 & 0,0 & 6,3 & $\mathbf{1 0 0}$ \\
\hline 5B & 28,9 & 36,8 & 5,3 & 0,0 & 10,5 & 0,0 & 18,4 & $\mathbf{1 0 0}$ \\
\hline 6A & 7,1 & 70,0 & 0,7 & 0,7 & 12,9 & 0,0 & 8,6 & $\mathbf{1 0 0}$ \\
\hline 6B & 11,8 & 57,4 & 10,1 & 1,3 & 6,8 & 0,8 & 11,8 & $\mathbf{1 0 0}$ \\
\hline $\mathbf{7}$ & 15,7 & 47,1 & 4,8 & 2,9 & 13,8 & 0,0 & 15,7 & $\mathbf{1 0 0}$ \\
\hline $\mathbf{8}$ & 15,2 & 57,6 & 6,1 & 3,0 & 9,1 & 0,0 & 9,1 & $\mathbf{1 0 0}$ \\
\hline
\end{tabular}

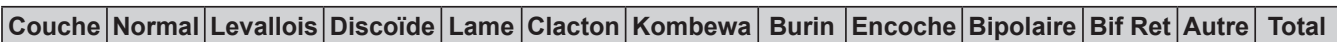

\begin{tabular}{|c|r|r|r|r|r|r|r|r|r|r|r|r|}
\hline 3A & 989 & 27 & 9 & 8 & 11 & 1 & 8 & 1 & 0 & 9 & 36 & $\mathbf{1 0 9 9}$ \\
\hline 3B & 1647 & 132 & 8 & 13 & 15 & 5 & 4 & 4 & 0 & 15 & 33 & $\mathbf{1 ~ 8 7 6}$ \\
\hline 4A & 96 & 10 & 3 & 0 & 1 & 0 & 0 & 2 & 0 & 16 & 6 & $\mathbf{1 3 4}$ \\
\hline 4B & 28 & 3 & 0 & 0 & 0 & 0 & 0 & 0 & 0 & 1 & 0 & $\mathbf{3 2}$ \\
\hline 4C & 340 & 32 & 4 & 7 & 2 & 2 & 0 & 6 & 1 & 11 & 8 & $\mathbf{4 1 3}$ \\
\hline 5A & 759 & 104 & 5 & 21 & 0 & 4 & 1 & 0 & 0 & 11 & 12 & $\mathbf{9 1 7}$ \\
\hline 5B & 326 & 46 & 0 & 0 & 0 & 2 & 0 & 0 & 2 & 6 & 1 & $\mathbf{3 8 3}$ \\
\hline 6A & 1093 & 240 & 5 & 14 & 4 & 9 & 2 & 0 & 0 & 10 & 23 & $\mathbf{1 4 0 0}$ \\
\hline 6B & 1093 & 223 & 10 & 7 & 5 & 4 & 2 & 0 & 2 & 8 & 31 & $\mathbf{1 3 8 5}$ \\
\hline $\mathbf{7}$ & 1940 & 89 & 12 & 10 & 6 & 12 & 2 & 1 & 0 & 3 & 19 & $\mathbf{2} \mathbf{0 9 4}$ \\
\hline $\mathbf{8}$ & 1137 & 175 & 3 & 22 & 1 & 0 & 2 & 2 & 2 & 10 & 7 & $\mathbf{1 3 6 1}$ \\
\hline Total & $\mathbf{9 4 4 8}$ & $\mathbf{1 0 8 1}$ & $\mathbf{5 9}$ & $\mathbf{1 0 2}$ & $\mathbf{4 5}$ & $\mathbf{3 9}$ & $\mathbf{2 1}$ & $\mathbf{1 6}$ & $\mathbf{7}$ & $\mathbf{1 0 0}$ & $\mathbf{1 7 6}$ & $\mathbf{1 1 0 9 4}$ \\
\hline
\end{tabular}

Tabl. XV - Compte des types d'éclats. 
Tabl. XVI - Compte typologique des différentes couches selon la liste-type de F. Bordes. Le matériel est issu des fouilles de H. Dibble et S. MacPherron.

\begin{tabular}{|c|c|c|c|c|c|c|c|c|c|c|c|c|c|c|}
\hline \multirow{2}{*}{ Type } & \multicolumn{13}{|c|}{ Couche } & \multirow{2}{*}{ ta } \\
\hline & 1 & 2 & $3 A$ & \begin{tabular}{|l|} 
\\
\end{tabular} & 4A & 4B & $4 C$ & 5A & $5 B$ & $6 \mathrm{~A}$ & $6 \mathrm{~B}$ & 7 & 8 & \\
\hline 1 & 10 & 6 & 16 & \begin{tabular}{l|l}
6 & 96 \\
\end{tabular} & 11 & 1 & 15 & 64 & 24 & 169 & \begin{tabular}{l|l}
9 & 126 \\
\end{tabular} & 59 & 122 & 720 \\
\hline 2 & 4 & 2 & 9 & \begin{tabular}{l|l|}
9 & 38 \\
\end{tabular} & 3 & 1 & 9 & 27 & 8 & \begin{tabular}{|l|l|}
3 & 70 \\
\end{tabular} & \begin{tabular}{l|l}
0 & 62 \\
\end{tabular} & 19 & 40 & 292 \\
\hline 3 & 3 & & 2 & 2 & & & 2 & 3 & 2 & & 2 & 1 & 3 & 20 \\
\hline 4 & & & & & & & & & & & & 2 & . & 3 \\
\hline 5 & 9 & 6 & 42 & \begin{tabular}{l|l}
2 & 21 \\
\end{tabular} & 3 & & 6 & 9 & 2 & 12 & \begin{tabular}{l|l}
2 & 23 \\
\end{tabular} & 25 & 13 & 171 \\
\hline 6 & & & & & & & 1 & 1 & & & 1 & & 5 & 8 \\
\hline 7 & & & & & & & & 2 & & & & & & 2 \\
\hline 9 & & & 7 & 5 & 4 & 1 & 8 & 12 & 10 & 16 & 5 & 19 & 20 & 107 \\
\hline 10 & 3 & & 4 & \begin{tabular}{l|l}
4 & 16 \\
\end{tabular} & 9 & 3 & 26 & 31 & 11 & 34 & 9 & 18 & 44 & 208 \\
\hline 11 & & & 1 & 4 & & & 2 & 1 & 1 & & & 4 & 5 & 18 \\
\hline 12 & & & & & & & & 1 & & 1 & 1 & 2 & & 4 \\
\hline 13 & & & & 3 & & & 2 & 2 & 1 & & & & 3 & 11 \\
\hline 14 & & & & & & & & & & & & 1 & 1 & 2 \\
\hline 15 & & & & 1 & 1 & & 4 & 1 & 2 & 1 & 1 & 2 & 7 & 19 \\
\hline 17 & & & & & & & & 1 & & & & 2 & 2 & 5 \\
\hline 18 & & & & & & & & 1 & & & & 2 & 3 & 6 \\
\hline 19 & & & & 2 & & 1 & 5 & 6 & 1 & 1 & 1 & 5 & 11 & 32 \\
\hline 20 & & & & & & & & & & 1 & 1 & & & 1 \\
\hline 21 & & & & & & 1 & 6 & 2 & 1 & 1 & 1 & & & 11 \\
\hline 22 & & 1 & & 2 & & & 2 & 3 & 1 & & & 2 & & 11 \\
\hline 23 & & & 1 & 1 & 5 & & 3 & 1 & & 1 & 1 & 3 & 3 & 18 \\
\hline 24 & & & & & & & & & & & & & 2 & 2 \\
\hline 25 & & & 1 & 1 & & & 1 & 1 & 1 & 5 & 3 & 3 & 1 & 17 \\
\hline 26 & & & & 2 & & & & & 1 & 1 & 2 & 2 & & 8 \\
\hline 27 & & & & & 1 & & 1 & & 1 & 1 & 1 & & & 4 \\
\hline 29 & & & & & & & & & & & 1 & & & 1 \\
\hline 30 & & & 1 & 2 & 1 & & & 1 & & 2 & 3 & 4 & & 14 \\
\hline 31 & & & & & & & & & 2 & 2 & 2 & 1 & 1 & 6 \\
\hline 32 & & 1 & 3 & 6 & & & 2 & 1 & 2 & 1 & 1 & 4 & 5 & 26 \\
\hline 33 & & 1 & & & & & & 1 & & 1 & 1 & 2 & 1 & 7 \\
\hline 34 & & & 2 & 3 & & & & 2 & 1 & & 2 & 3 & & 13 \\
\hline 35 & & & & 3 & & & & 1 & & 1 & 1 & & & 6 \\
\hline 36 & 1 & 2 & 5 & 1 & & & & 1 & & & & & 1 & 11 \\
\hline 37 & 2 & & 1 & 1 & & & & 1 & & 2 & 2 & 1 & & 10 \\
\hline 38 & 10 & 3 & 36 & \begin{tabular}{l|l}
6 & 60 \\
\end{tabular} & 5 & 1 & 11 & 45 & 20 & 52 & \begin{tabular}{l|l}
2 & 42 \\
\end{tabular} & 38 & 46 & 369 \\
\hline 39 & & & & 1 & & & & 1 & & & 1 & & & 3 \\
\hline 40 & 2 & & 1 & 4 & & & & & 2 & 3 & 2 & 4 & 2 & 20 \\
\hline 42 & 10 & 9 & 43 & \begin{tabular}{l|l}
3 & 73 \\
\end{tabular} & 1 & & 2 & 13 & 7 & 42 & \begin{tabular}{l|l}
2 & 30
\end{tabular} & 49 & 15 & 294 \\
\hline 43 & 16 & 2 & 45 & \begin{tabular}{l|l}
5 & 72 \\
\end{tabular} & 2 & & 8 & 3 & 4 & 28 & \begin{tabular}{l|l}
8 & 27 \\
\end{tabular} & 52 & 10 & 269 \\
\hline 44 & & & 1 & 1 & & & 1 & & & & 2 & 2 & & 7 \\
\hline 45 & & 2 & & & & & & 2 & & 3 & 1 & 2 & 1 & 11 \\
\hline 48 & 19 & 14 & 35 & \begin{tabular}{l|l}
5 & 96 \\
\end{tabular} & 4 & 1 & 5 & 18 & 136 & 96 & \begin{tabular}{l|l}
6 & 188 \\
\end{tabular} & \begin{tabular}{l|l|l}
3 & 1600 \\
\end{tabular} & 71 & 2283 \\
\hline 50 & & & & 1 & & & & & & & & & & 1 \\
\hline 51 & & & & 2 & & & 1 & & & & & 2 & & 5 \\
\hline 54 & & 1 & 2 & \begin{tabular}{l|l}
2 & 12 \\
\end{tabular} & 1 & 1 & 2 & 3 & 2 & 3 & 6 & 2 & 1 & 36 \\
\hline 56 & & & & 1 & & & & & & & 1 & & & 2 \\
\hline 57 & & & & & & & & & & & & 1 & & 1 \\
\hline 58 & & & & & & & & & & & 1 & & & 1 \\
\hline 59 & 1 & & & 1 & & & & & & & & & & 2 \\
\hline 61 & & 1 & & & & & 1 & & & & & & & 2 \\
\hline 62 & 2 & & 5 & 1 & & & & & 2 & 2 & 6 & & & 18 \\
\hline 64 & 9 & & 7 & 9 & 1 & & 1 & 7 & 4 & 51 & \begin{tabular}{l|l}
1 & 58 \\
\end{tabular} & 38 & 14 & 199 \\
\hline otal & 101 & 51 & 270 & \begin{tabular}{l|l}
0 & 544
\end{tabular} & 52 & 11 & 127 & 269 & 249 & 603 & \begin{tabular}{l|l}
3 & 609
\end{tabular} & \begin{tabular}{l|l}
1975 \\
\end{tabular} & 454 & 5315 \\
\hline
\end{tabular}

Tabl. XVII - Rapport support/nucléus et outil/nucléus.

\begin{tabular}{|c|c|c|c|c|c|c|c|c|c|c|}
\hline \multicolumn{10}{|c|}{ Couche } \\
\hline 3A & 3B & 4A & 4B & 4C & 5A & 5B & 6A & 6B & 7 & 8 \\
\hline
\end{tabular}

\begin{tabular}{|l|l|l|l|l|l|l|l|l|l|l|l|l|}
\hline Total & 1638 & 2938 & 240 & 49 & 650 & 1452 & 718 & 2497 & 2490 & 4207 & 2590 \\
\hline
\end{tabular} \begin{tabular}{|l|l|l|l|l|l|l|l|l|l|l|l|}
\hline Support /nucléus & 22,0 & 20,7 & 33,0 & 15,5 & 20,4 & 19,0 & 8,5 & 7,8 & 4,7 & 7,6 & 32,1 \\
\hline Outis/clats & 0,10 & 0,08 & 0,17 & 0,15 & 0,14 & 0,07 & 0,12 & 0,10 & 0,07 & 0,07 & 0,07 \\
\hline
\end{tabular} \begin{tabular}{lllllllllll|l|l|l|l|}
\hline Outils/éclats & 0,10 & 0,08 & 0,17 & 0,15 & 0,14 & 0,07 & 0,12 & 0,10 & 0,07 & 0,07 & 0,07 \\
\hline
\end{tabular}

Tabl. XVIII - Moyenne pondérale de certains types d'outils ou d'objets.

\begin{tabular}{|c|c|c|c|c|}
\hline Couche & Racloirs & Encoches & Autres outils & Éclats \\
\hline 3A & 15,0 & 12,4 & 12,5 & 7,7 \\
\hline 3B & 10,0 & 11,5 & 8,2 & 6,9 \\
\hline 4A & 36,0 & 4,8 & 10,8 & 9,4 \\
\hline 4B & 23,0 & 12,0 & 8,5 & 10,0 \\
\hline 4C & 26,3 & 19,2 & 11,3 & 7,3 \\
\hline 5A & 16,4 & 10,2 & 9,1 & 7,4 \\
\hline 5B & 20,6 & 14,0 & 8,5 & 9,5 \\
\hline 6A & 23,2 & 16,3 & 7,9 & 9,8 \\
\hline 6B & 12,4 & 9,8 & 7,6 & 7,4 \\
\hline 7 & 12,9 & 12,2 & 8,3 & 9,1 \\
\hline $\mathbf{8}$ & 11,9 & 10,1 & 7,1 & 6,2 \\
\hline
\end{tabular}

Tabl. XIX - Altération des objets lithiques: $\boldsymbol{a}$, en nombre; $\boldsymbol{b}$, en pourcentage.

\begin{tabular}{|c|r|r|r|r|r|r|r|r|}
\hline Couche & Frais & $\begin{array}{c}\text { Légère } \\
\text { patine }\end{array}$ & Moucheté & $\begin{array}{c}\text { Forte } \\
\text { patine }\end{array}$ & Blanc & Brûlé & Autre & Total \\
\hline 3A & 626 & 311 & 160 & 293 & 75 & 37 & 6 & $\mathbf{1 5 0 8}$ \\
\hline 3B & 1218 & 590 & 232 & 544 & 136 & 31 & 16 & $\mathbf{2 7 6 7}$ \\
\hline 4A & 787 & 59 & 16 & 36 & 28 & 3 & 1 & $\mathbf{2 2 1}$ \\
\hline 4B & 11 & 16 & 3 & 16 & 2 & 0 & 0 & $\mathbf{4 8}$ \\
\hline 4C & 200 & 140 & 91 & 131 & 51 & 8 & 2 & $\mathbf{6 2 3}$ \\
\hline 5A & 475 & 290 & 315 & 204 & 63 & 29 & 4 & $\mathbf{1 3 8 0}$ \\
\hline 5B & 210 & 164 & 79 & 144 & 54 & 13 & 4 & $\mathbf{6 6 8}$ \\
\hline 6A & 717 & 483 & 297 & 394 & 187 & 190 & 2 & $\mathbf{2 2 7 0}$ \\
\hline 6B & 757 & 448 & 215 & 405 & 202 & 251 & 24 & $\mathbf{2 3 0 2}$ \\
\hline $\mathbf{7}$ & 746 & 905 & 338 & 801 & 397 & 466 & 32 & $\mathbf{3 6 8 5}$ \\
\hline $\mathbf{8}$ & 348 & 491 & 144 & 353 & 318 & 773 & 20 & $\mathbf{2 4 4 7}$ \\
\hline Total & $\mathbf{5 3 8 6}$ & $\mathbf{3 8 9 7}$ & $\mathbf{1 8 9 0}$ & $\mathbf{3 3 2 1}$ & $\mathbf{1 5 1 3}$ & $\mathbf{1 8 0 1}$ & $\mathbf{1 1 1}$ & $\mathbf{1 7 9 1 9}$ \\
\hline
\end{tabular}

\begin{tabular}{|c|c|c|c|c|c|c|c|c|c|}
\hline Couche & Frais & $\begin{array}{l}\text { Légère } \\
\text { patine }\end{array}$ & $\begin{array}{l}\text { Forte } \\
\text { patine }\end{array}$ & Patiné & Moucheté & Blanc & Brûlé & Autre & Total \\
\hline $3 A$ & 41,5 & 20,6 & 19,4 & 40,0 & 10,6 & 5,0 & 2,5 & 0,4 & 100 \\
\hline 3B & 44,0 & 21,3 & 19,7 & 41,0 & 8,4 & 4,9 & 1,1 & 0,6 & 100 \\
\hline $4 \mathrm{~A}$ & 35,3 & 26,7 & 16,3 & 43,0 & 7,2 & 12,7 & 1,4 & 0,5 & 100 \\
\hline 4B & 22,9 & 33,3 & 33,3 & 66,7 & 6,3 & 4,2 & 0,0 & 0,0 & 100 \\
\hline $4 \mathrm{C}$ & 32,1 & 22,5 & 21,0 & 43,5 & 14,6 & 8,2 & 1,3 & 0,3 & 100 \\
\hline $5 A$ & 34,4 & 21,0 & 14,8 & 35,8 & 22,8 & 4,6 & 2,1 & 0,3 & 100 \\
\hline $5 B$ & 31,4 & 24,6 & 21,6 & 46,2 & 11,8 & 8,1 & 1,9 & 0,6 & 100 \\
\hline $6 \mathrm{~A}$ & 31,6 & 21,3 & 17,4 & 38,7 & 13,1 & 8,2 & 8,4 & 0,1 & 100 \\
\hline $6 B$ & 32,9 & 19,5 & 17,6 & 37,1 & 9,3 & 8,8 & 10,9 & 1,0 & 100 \\
\hline 7 & 20,2 & 24,6 & 21,7 & 46,3 & 9,2 & 10,8 & 12,6 & 0,9 & 100 \\
\hline 8 & 14,2 & 20,1 & 14,4 & 34,5 & 5,9 & 13,0 & 31,6 & 0,8 & 100 \\
\hline
\end{tabular}



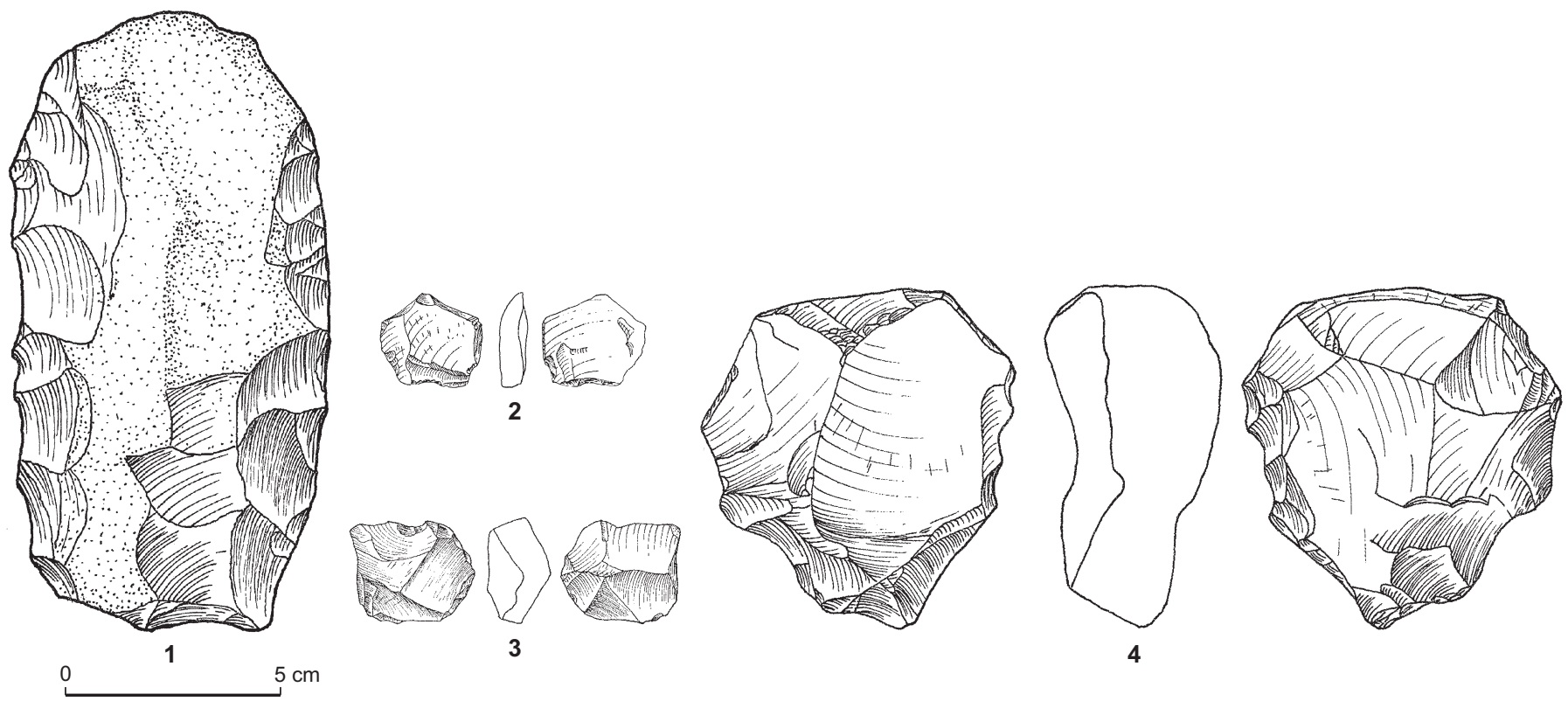

Fig. 22 - Couche 7: 1, racloir double convexe; 2, éclat à retouche alterne; 3 et 4 fragment de nucléus (dessin: T. Tsenova).

Tabl. XX - Compte total des vestiges lithiques et osseux, volume de sédiment, ratio entre silex et os et densité de silex et d'os par litre de sédiments.

\begin{tabular}{|l|c|c|c|c|c|c|c|c|c|c|c|}
\cline { 2 - 13 } \multicolumn{1}{c|}{} & \multicolumn{10}{c|}{ Couche } \\
\cline { 2 - 13 } \multicolumn{1}{c|}{} & 3A & 3B & 4A & 4B & 4C & 5A & 5B & 6A & 6B & 7 & 8 \\
\hline Total silex & 1638 & 2938 & 240 & 49 & 650 & 1452 & 718 & 2497 & 2490 & 4207 & 2590 \\
\hline Total os & 746 & 956 & 811 & 412 & 3828 & 2637 & 1033 & 4917 & 5342 & 340 & 1731 \\
\hline Volume des sédiments (litre) & 1148 & 791 & 2443 & 742 & 735 & 707 & 1435 & 2163 & 2534 & 791 & 1862 \\
\hline Silex/Os & 2,2 & 3,07 & 0,30 & 0,19 & 0,17 & 0,55 & 0,70 & 0,51 & 0,47 & 12,4 & 1,50 \\
\hline Silex/litre de sédiment & 1,43 & 3,71 & 0,10 & 0,07 & 0,88 & 2,05 & 0,50 & 1,15 & 0,98 & 5,32 & 1,39 \\
\hline Os/litre de sédiment & 0,65 & 1,21 & 0,33 & 0,56 & 5,21 & 3,73 & 0,72 & 2,27 & 2,11 & 0,43 & 0,93 \\
\hline
\end{tabular}

De plus, le pourcentage d'outils est le plus bas de toute la séquence et le nombre de supports par nucléus s'élève à environ 32, seul celui de la couche 4A est légèrement supérieur avec 33 supports par nucléus (tabl. XVII).

\section{LA COUCHE 7}

La couche 7 a livré la plus grande quantité de matériel lithique, aussi bien en nombre que par rapport au volume de sédiments (fig. 22, tabl. XX). Il s'agit probablement d'un lobe de solifluxion, ce qui explique l'aspect roulé et émoussé. En fait, $83 \%$ des pièces sont des «éclats à retouche alterne et abrupte» (type 48, tabl. XVI). Comme les artefacts sont fortement endommagés, toute étude typologique ou technologique significative de cet assemblage lithique est impossible.

\section{LA COUCHE 6}

La couche 6 a un indice Levallois relativement élevé avec 43,3\% (fig. 23-26). L'indice de racloir est fort et dominé par les latéraux rectilignes et convexes. Il y a également une augmentation notable du nombre d'encoches et de denticulés.

L'élément le plus important de cette industrie lithique est l'attention portée à la production de petits éclats (Dibble, McPherron, 2006 et 2007) et par conséquent, la présence de très petits nucléus Levallois et un nombre élevé de produits Kombewa. Ces éléments déjà observés par F. Bordes dans les couches correspondantes (J3A-J3C) l'avait conduit à qualifier ce Moustérien typique d'Asinipodien ajoutant ainsi le Pech de l'Azé IV à la longue liste des sites éponymes du Périgord. 


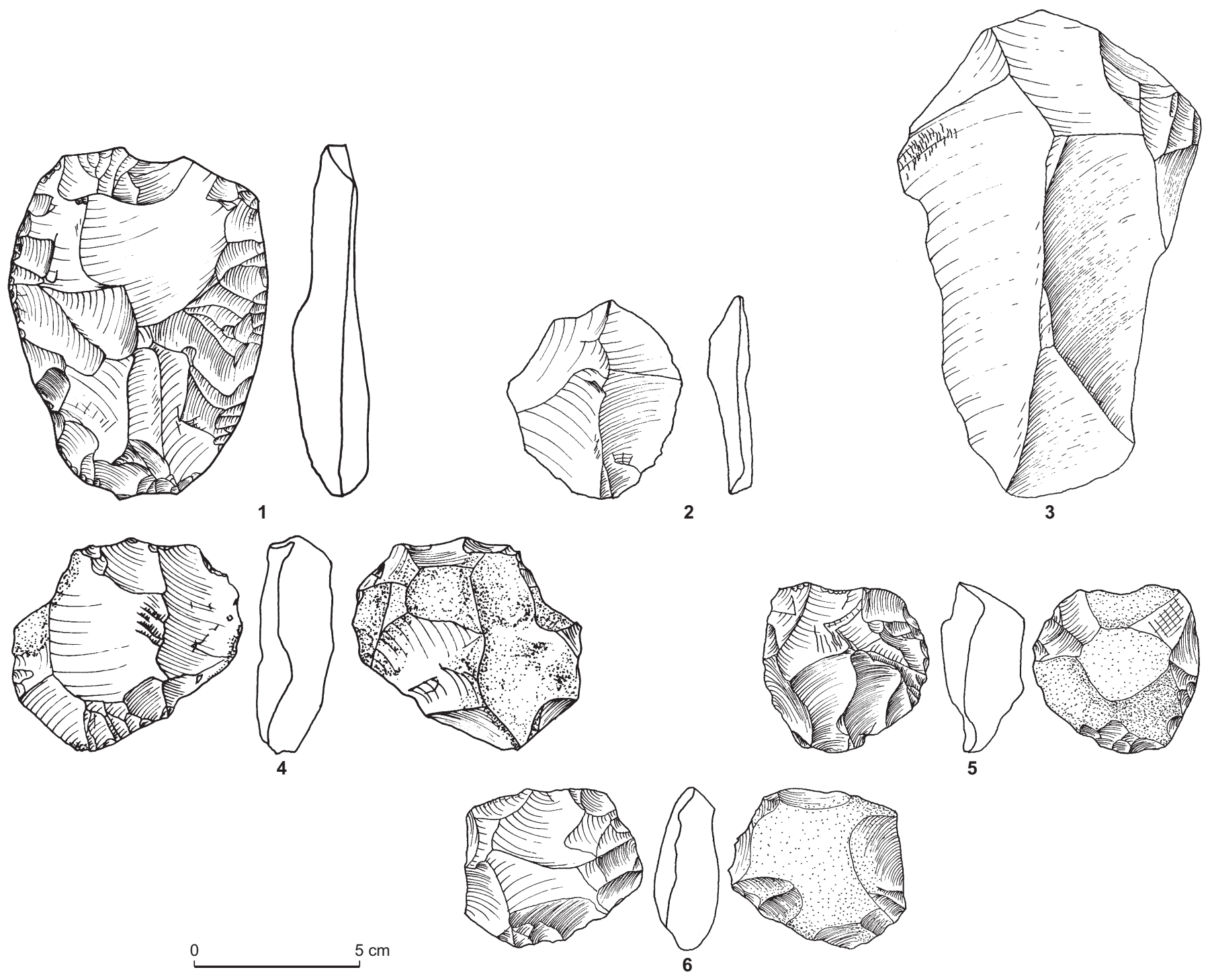

Fig. 23 - Couche 6A: 1, racloir double; 2 et 3, éclat Levallois; 4, 5 et 6 nucléus Levallois (dessin: T. Tsenova).

\section{LA GOUGHE 5}

Comme la couche précédente, celle-ci a un fort indice Levallois et présente une augmentation significative de produits Levallois, une tendance amorcée dans les niveaux sous-jacents (à l'exclusion de la couche 7) (fig. 27 et 28). F. Bordes a attribué les niveaux correspondants (J1 à J3) au Moustérien typique, ce qui est corroboré dans nos données par un indice relativement élevé en racloirs (dominé par les types simples) et une faible fréquence des encoches et denticulés.

\section{LA COUCHE 4}

Elle a livré moins d'objets lithiques (fig. 29-31). En termes d'artefacts et de volume de sédiments fouillés, elle arrive en second après le niveau 6B. Moins de 1000 objets lithiques ont été recueillis dans un peu moins de $2,5 \mathrm{~m}^{3}$. Il est tout à fait évident que les occupations au cours de cette période ont été plus sporadiques, avec des hiatus visibles entre des périodes aux concentrations plus denses en artefacts. 
La couche 4 est subdivisée en trois sous-unités selon la nature des sédiments. Toutes sont riches en racloirs, mais à des niveaux différents. Le niveau le plus élevé, 4A, présente bien plus un aspect de Moustérien de type Quina, avec des formes plus épaisses et transverses, que Levallois. La proportion d'outils par rapport au nombre d'éclats est significativement plus élevée, comme le choix des plus grands supports pour être transformés en racloirs (tabl. XX). Il est clair que dans ces niveaux (voir supra, p. 26), l'utilisation de techniques de fouilles plus précises a permis d'isoler les différentes lentilles d'une manière qui n'était pas possible pour F. Bordes, ce qui explique sans doute pourquoi il n'a pas été en mesure d'identifier un horizon Quina clair sur le site (McPherron et al., 2005).

\section{LA COUCHE 3}

La couche 3 n'est pas facilement attribuable à un faciès moustérien spécifique (fig. 32-34). F. Bordes a découvert un certain nombre de bifaces dans les niveaux correspondants et a attribué les industries au Moustérien de tradition acheuléenne. Nous avons retrouvé un biface rudimentaire dans le niveau 3B, et la présence de burins et de couteaux à dos est plus élevée que toute autre couche, ce qui nous conduit provisoirement à la conclusion de F. Bordes. Toutefois, les autres types d'outils caractéristiques de ce faciès, comme les couteaux à dos typiques et atypiques, ne sont pas fortement représentés, ni en 3A, ni en 3B, alors que les couteaux à dos naturel le sont relativement. Les couches 3A et 3B ont également un nombre élevé de pointes pseudo-Levallois. Ces deux types d'éclats semblent avoir été un centre d'intérêt de la production lithique pendant ces occupations (Sandgathe, 2005). La couche 3 présente également le pourcentage le plus élevé en encoches et denticulés, dont certains sont exceptionnellement réguliers. En effet, on peut dire que la couche 3 est semblable à un MTA pauvre en biface.

Il est intéressant de noter que les assemblages du Pech de l'Azé IV sont très différents dans leur composition globale des niveaux MTA du Pech de l'Azé I voisin (Soressi, 2002; Soressi et al., 2008). Bien qu'il existe quelques éclats bifaciaux fins au Pech de l'Azé IV, leur abondance est nettement inférieure à celle observée au Pech I, de même que les bifaces sont moins abondants et moins bien faits. Cette variabilité entre les sites et le nombre relativement faible des outils typiques du MTA peuvent être expliqués par le fait qu'au moment de l'occupation, la grotte était presque remplie et la zone fouillée principalement située à l'aplomb de
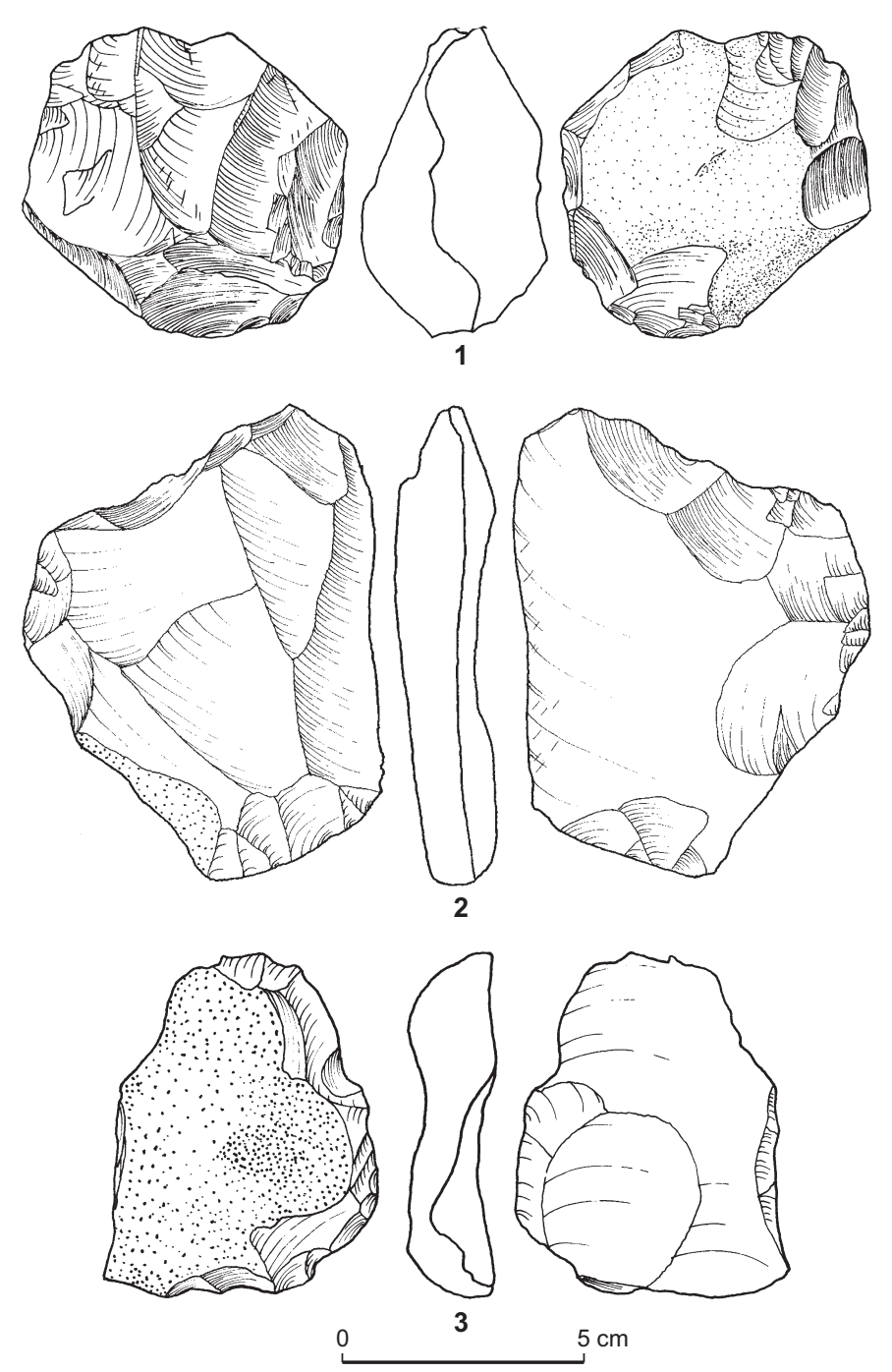

Fig. 24 - Couche 6A: 1, nucléus à une surface de débitage; 2, pièce tronquée-facettée; 3, nucléus Kombewa (dessin: T. Tsenova).

la voûte, en particulier dans la couche 3A, peut-être à la périphérie de la zone principale d'occupation. En l'absence de fouilles plus amples, il est difficile de tester cette hypothèse.

\section{LES COUCHES 1 ET 2}

Ces couches, qui coiffent la séquence du site, sont largement composées de sédiments pléistocènes redéposés au cours de l'Holocène. Elles comprennent également une matière organique importante résultant des processus de développement du sol actuel. Les composantes lithiques et fauniques représentent un mélange d'éléments paléolithiques perturbés et de quelques artefacts récents (un très 

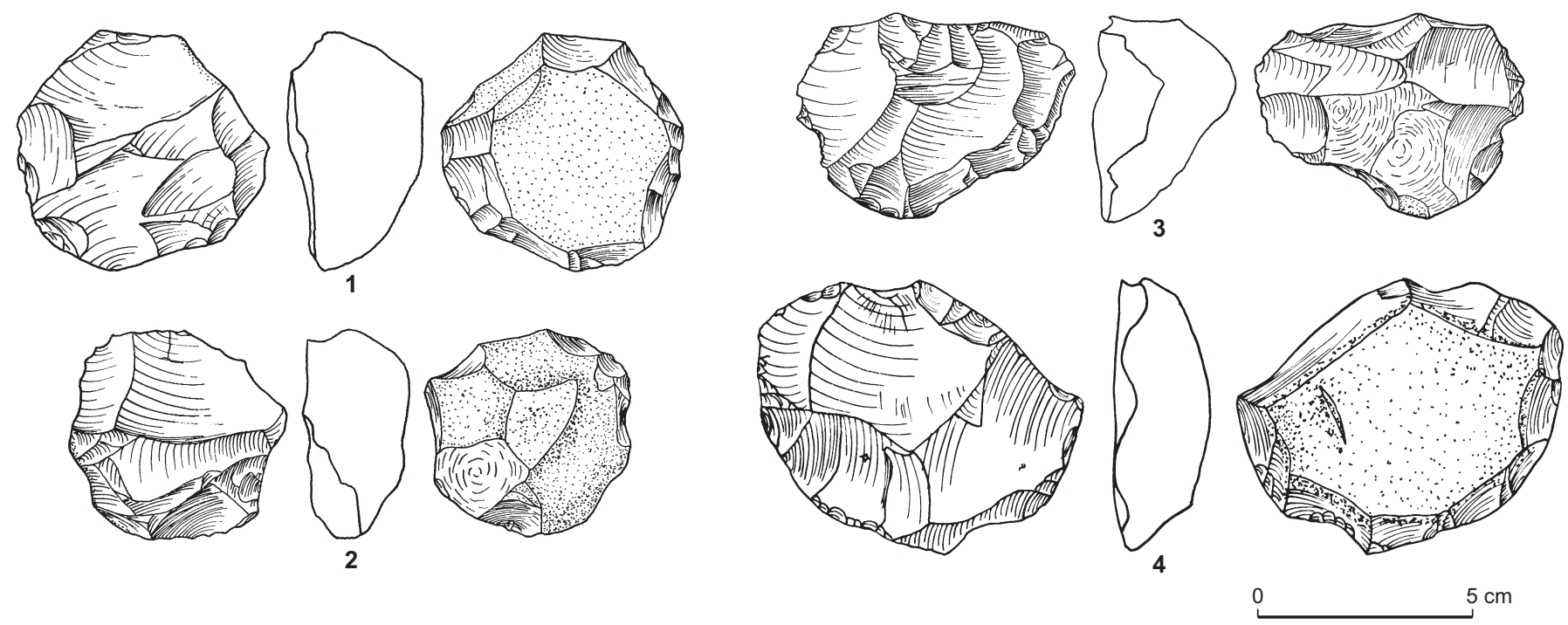

Fig. 25 - Couche 6B: nucléus à une surface de débitage (dessin: T. Tsenova).
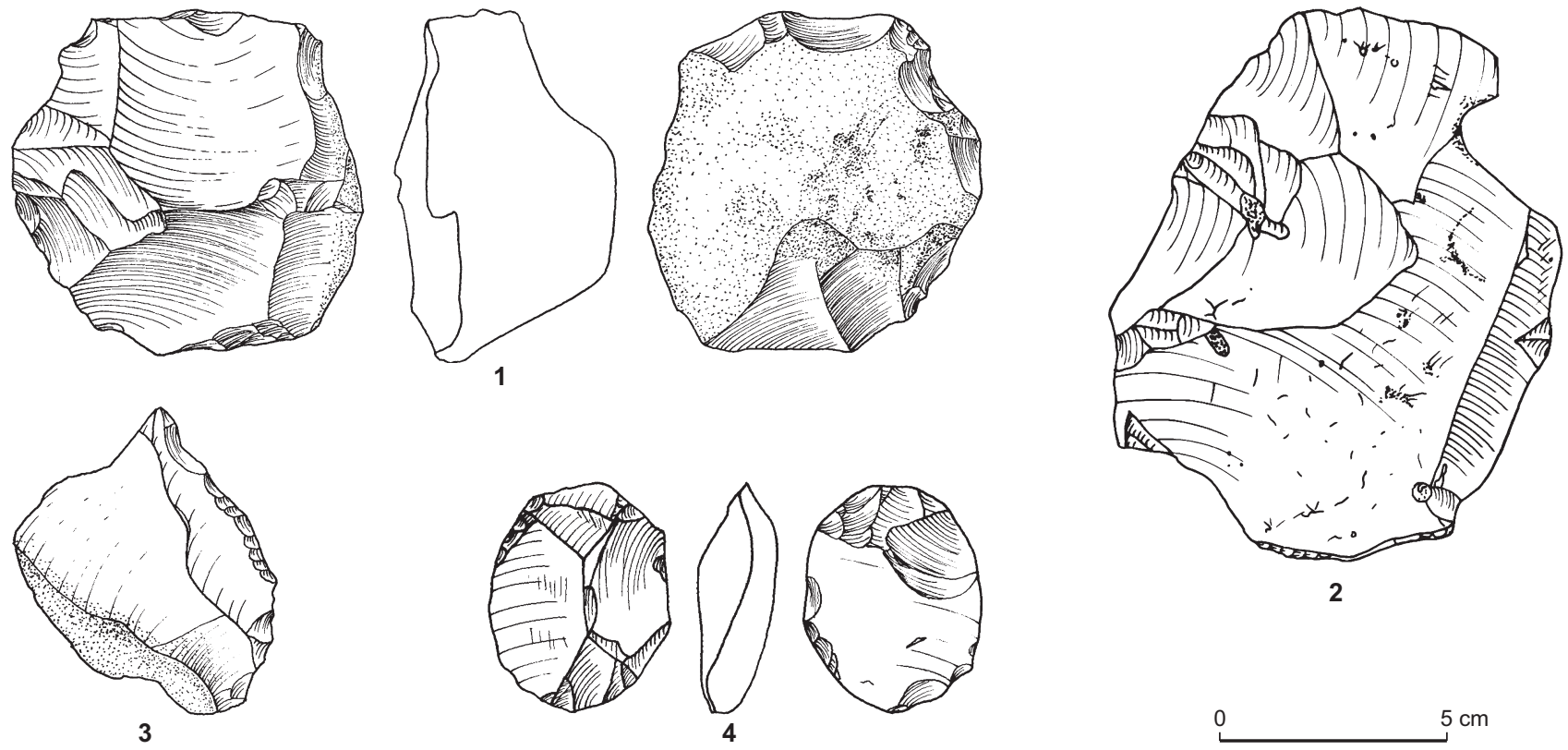

Fig. 26 - Couche 6B: 1, nucléus Levallois; 2, éclat Levallois; 3, bec à retouche alterne; 4, pièce tronquée facettée (dessin: T. Tsenova).

petit nombre d'objets historiques, de la période médiévale ou postérieure). La couche 1 est divisée en quatre sousunités selon la nature des sédiments, tandis que la couche 2 est une petite dépression formée par l'action de l'eau dans une zone limitée. Le faible nombre d'objets archéologiques et le degré de perturbation de la couche 1 signifient que les données apportées par cette dernière ne sont pas particulièrement utiles.

\section{QUELQUES COMPARAISONS ENTRE NIVEAUX}

En terme de technologie de production d'éclats basiques, il y a une nette tendance à la baisse de l'utilisation de la technique Levallois et de l'indice de facettage, souvent associés à travers la séquence (fig. 35a). Les indices Levallois sont plus élevés dans les couches de l'Asinipodien (6A et $6 \mathrm{~B})$. 

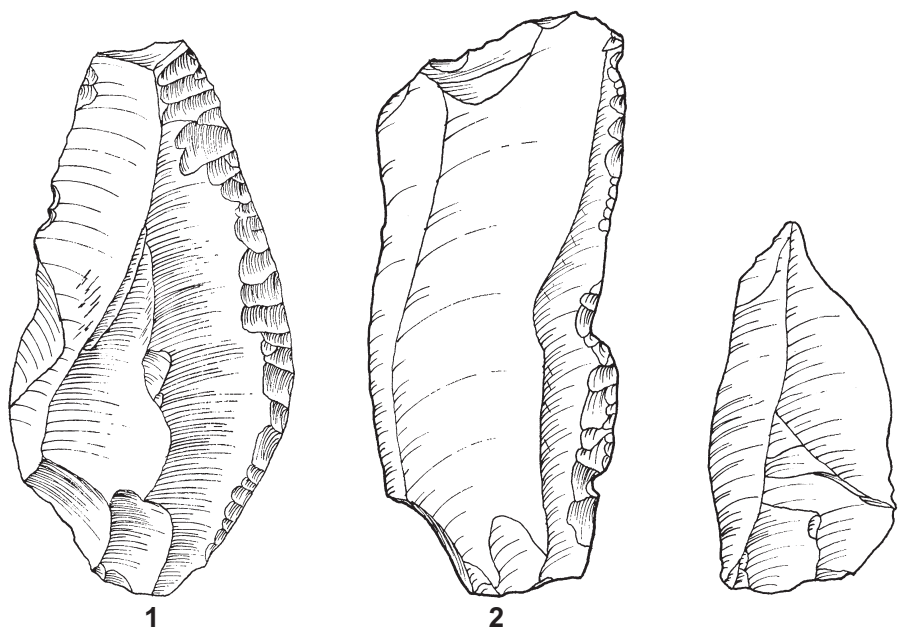

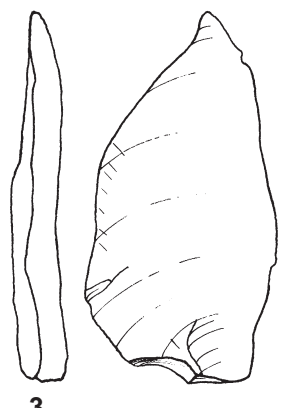

3

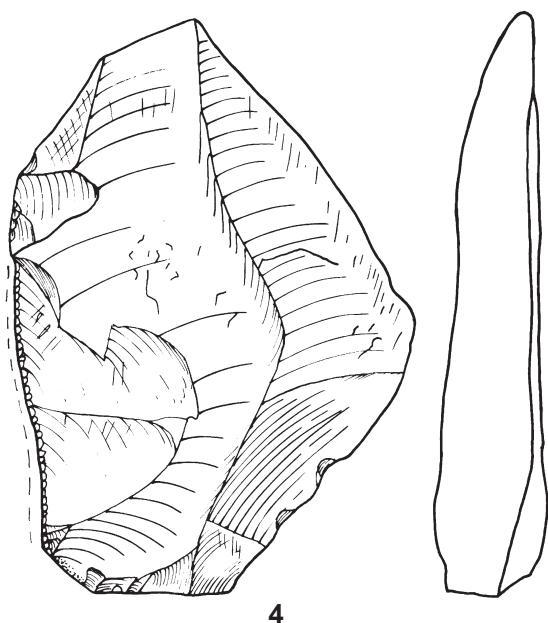

4
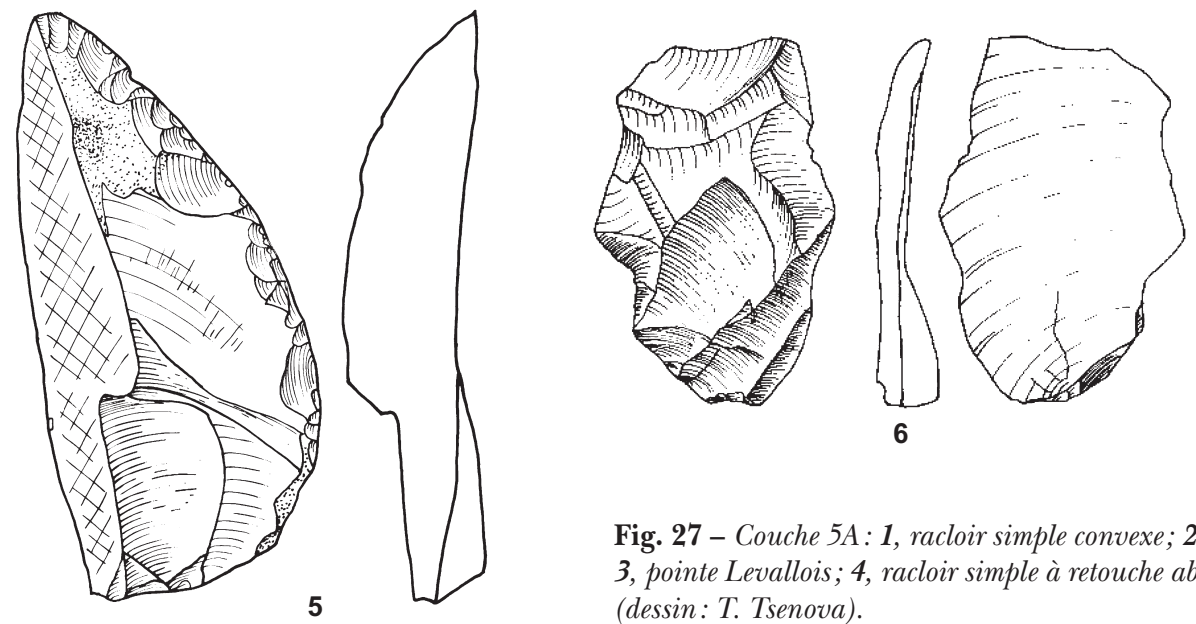

$5 \mathrm{~cm}$

Fig. 27 - Couche 5A: 1, racloir simple convexe; 2, racloir simple convexe sur éclat Levallois; 3, pointe Levallois; 4 , racloir simple à retouche abrupte; 5 , racloir simple convexe; 6, éclat (dessin: T. Tsenova).

Cela apparaît souvent dans les sites moustériens français (Dibble, 1988, 1991, 1995 et 1997; Rolland, Dibble, 1990; Dibble, Rolland, 1992; Mellars, 1996; Roth, Dibble, 1998), dû soit à une diminution des matières premières locales, soit à des changements dans l'utilisation du site. Toutefois, s'il s'agit de ce dernier cas, il est surprenant qu'il y ait si peu d'exemples de changements brusques ou de fluctuations dans un sens ou dans l'autre. Ces mêmes arguments pourraient être utilisés contre une explication «culturelle» de cette variabilité.

Il existe aussi un net changement dans l'utilisation de la technique Kombewa et la production de pièces tronquées et facettées (fig. 35b). Ces deux dernières ont été interprétées comme le reflet de la production délibérée de petits éclats, le plus souvent non retouchés (Dibble, McPherron, 2006 et $2007)^{8}$. Il existe un lien évident entre elles, qui atteint son

8. La production de petits éclats est pour certains chercheurs une caractéristique des supports du Paléolithique moyen (Bourguignon apogée dans la couche 6 où elles sont associées à de petits nucléus et éclats Levallois spécifiques de l'Asinipodien. Pour ce qui est de la technique Kombewa, sa diminution progressive de la couche $6 \mathrm{~A}$ à $3 \mathrm{~A}$ est marquée par une absence complète dans les couches $4 \mathrm{~A}$ et 4B.

La production de racloirs varie de façon significative dans la séquence, et atteint son apogée lors des occupations sporadiques des couches 4A-4C (fig. 36). L'utilisation de la technique Levallois augmente au fur et à mesure que l'on descend dans la séquence, et la production de racloirs diminue. En fait, durant l'Asinipodien du niveau 6B, la production de racloirs est à des taux faibles, identiques à ceux des niveaux les plus hauts dans la stratigraphie. D'autre

et al., 2004). Ces éclats sont rarement retouchés et sont produits selon des méthodes différentes dans les industries Levallois, Quina et discoïde. 

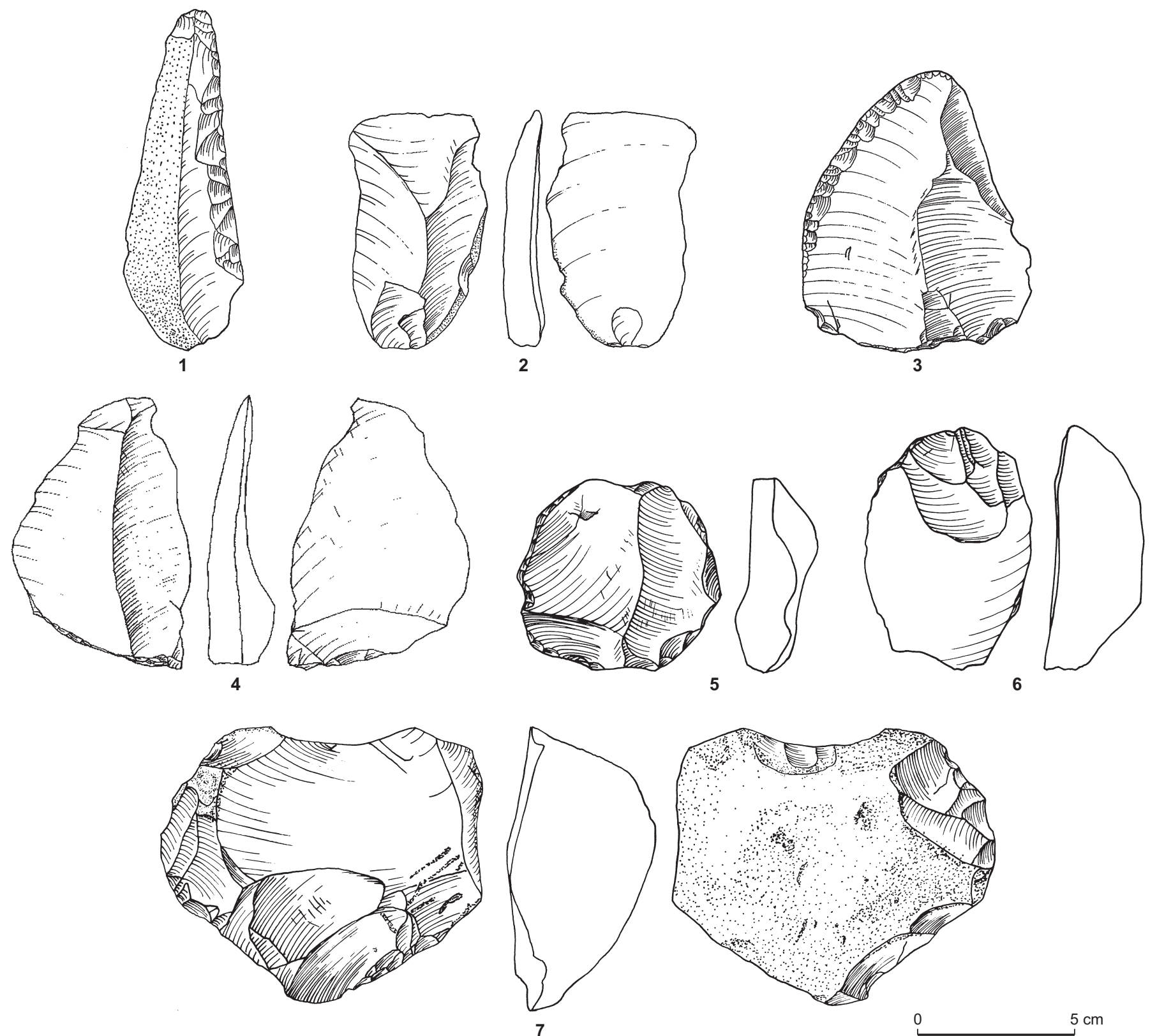

$5 \mathrm{~cm}$

Fig. 28 - Couche 5B: 1, racloir simple concave sur éclat à dos naturel; 2, éclat Levallois; 3, troncature; 4, racloir simple convexe; 5, nucléus à une surface de débitage; 6, nucléus sur éclat; 7, nucléus Levallois (dessin: T. Tsenova).

part, le niveau 8 , qui constitue la première occupation du site, a un indice Levallois un peu faible et un indice de racloir légèrement élevé.

Il y a plusieurs aspects de l'assemblage lithique qui se rapprochent d'autres processus observés sur le site. Deux sont des aspects comportementaux importants durant l'occupation du site. Tout d'abord, il y a une relation claire et directe entre le nombre de restes fauniques récupérés et le pourcentage de racloirs, atteignant tous les deux un pic dans les niveaux 4 (fig. 37). Le second aspect a trait à l'utilisation du feu, en particulier dans les couches inférieures, où le silex et le calcaire brûlés sont présents à des fréquences beaucoup plus élevées. L'association entre les foyers visibles et la chauffe du matériel lithique, conséquence secondaire, montre que l'absence de foyers dans les niveaux supérieurs n'est pas le reflet de la préservation (fig. 38). 

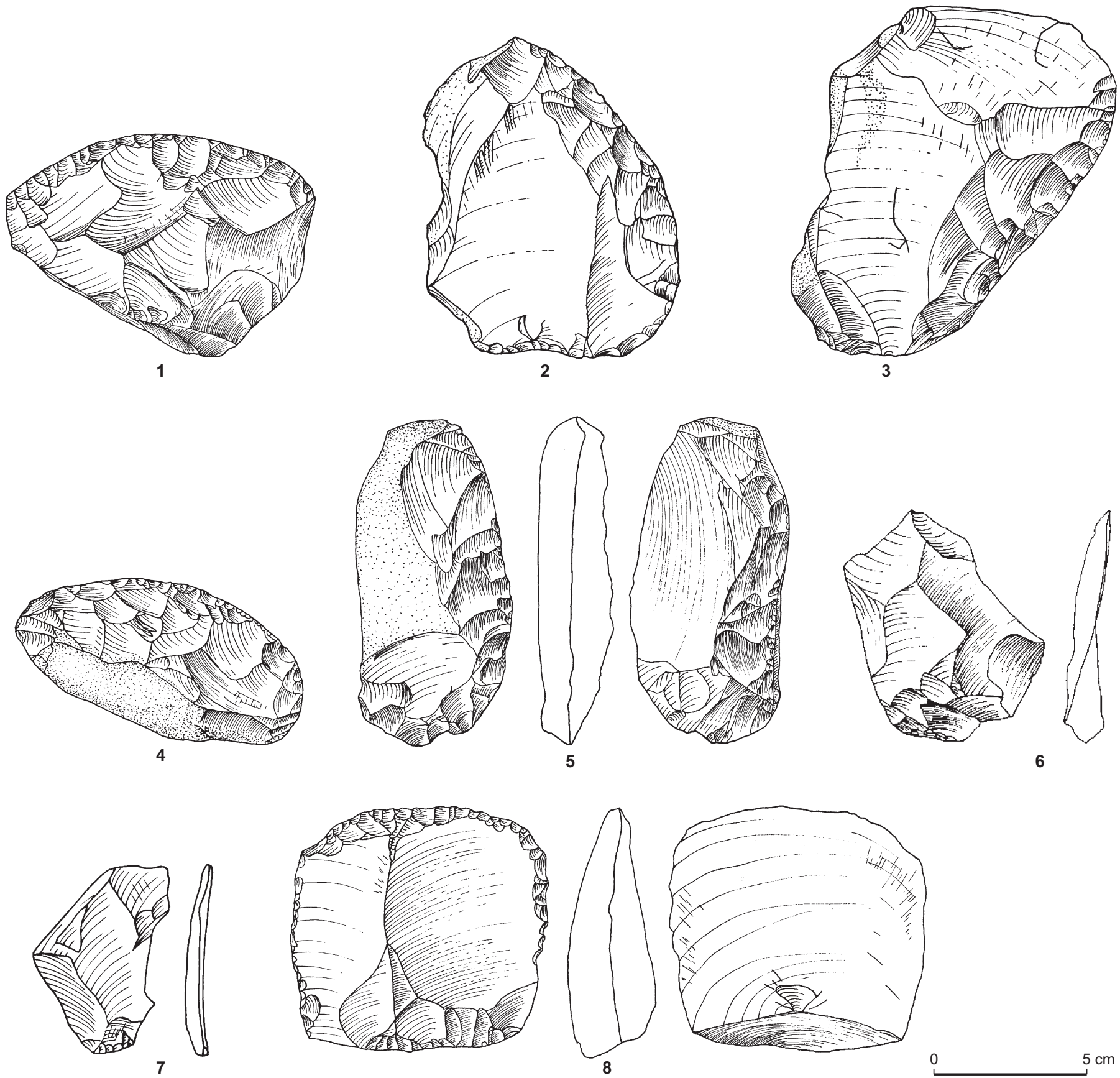
$5 \mathrm{~cm}$

Fig. 29 - Couche 4A: 1, 4 et 8, racloir transversal convexe; 2, racloir simple convexe; 4, racloir simple droit; 5, racloir à dos aminci; 6 et 7 , éclat (dessin: T. Tsenova). 

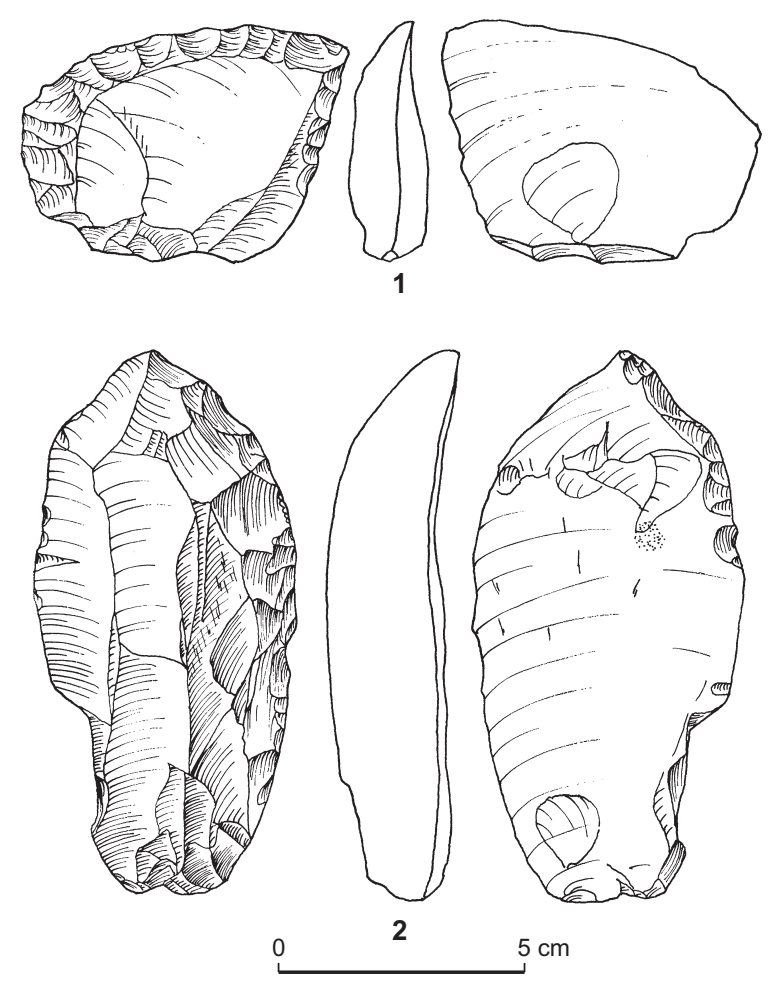

Fig. 30 - Couche 4B: 1, racloir déjeté; 2, racloir simple convexe (dessin: T. Tsenova).
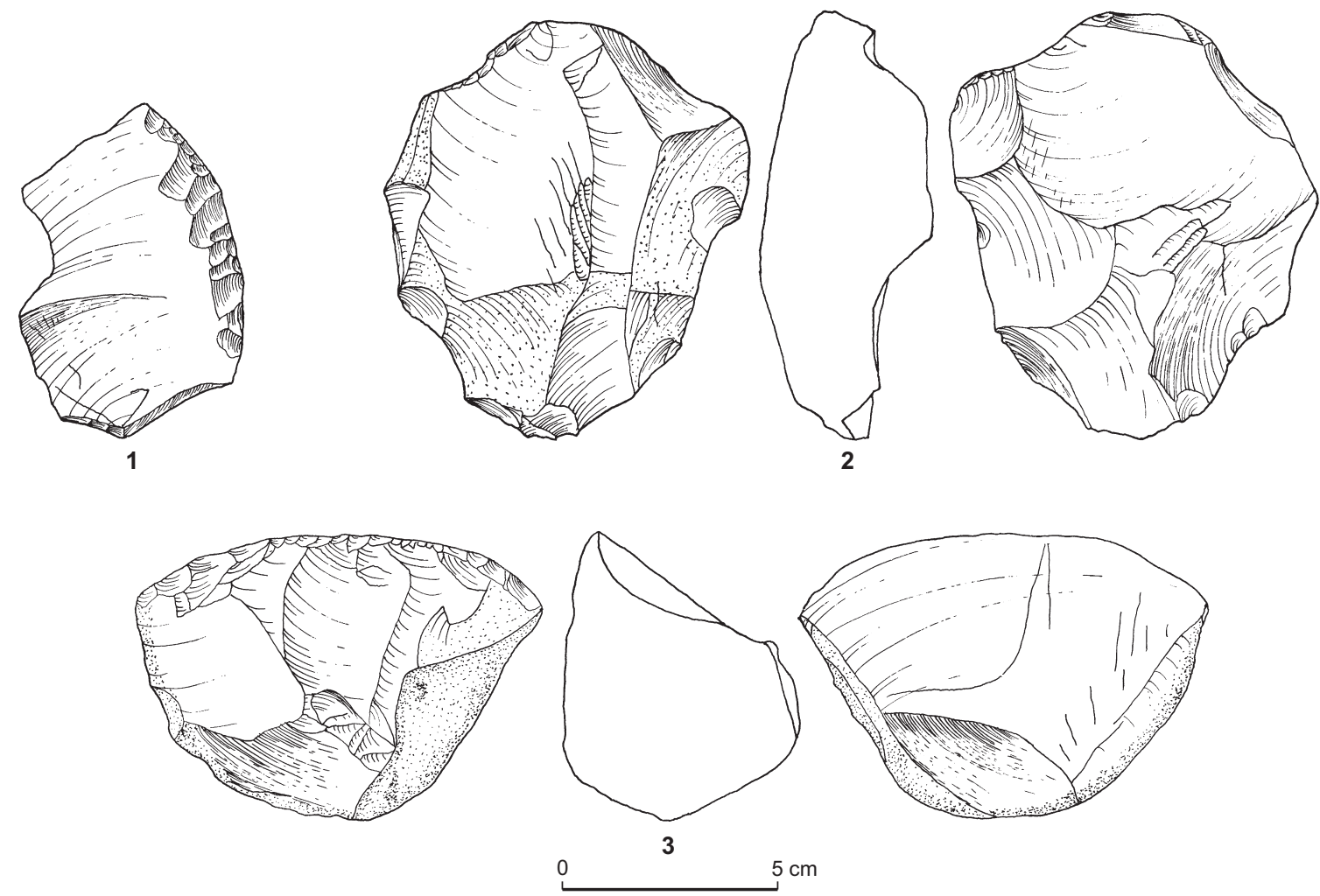

Fig. 31 - Couche 4C: 1, racloir sur face plane; 2, racloir transversal convexe; 3, nucléus Levallois (dessin: T. Tsenova). 
Il existe certains caractères évidents dans les assemblages du Pech de l'Azé IV suivant des schémas qui ont été remarqués ailleurs. Il y a par exemple une sélection claire des plus grandes pièces pour la retouche, ce qui a été signalé dans un certain nombre de sites moustériens dans les environs. Cependant, il est intéressant de noter que la production claire et délibérée de petits éclats dans les niveaux asinipodiens montre que, dans l'ensemble, la taille de l'objet n'était pas une caractéristique nécessaire des pièces jugées utiles.

\section{LES SURFACES D'OCGUPATION (5A1 ET 6A1)}

Hormis les structures de combustion, une des découvertes les plus intéressantes de la fouille fut la mise au jour de deux surfaces d'occupation scellées par deux épisodes d'effondrement du toit de la grotte (tabl. V). Ces surfaces d'occupation ont été découvertes lors de l'enlèvement d'environ deux mètres de blocs d'effondrement dans les carrés G14, H14, G13, H13, G12 et H12. À première vue, cet épisode semble correspondre à un événement unique et massif (fig. 11). Cependant, un examen plus attentif de la coupe révèle des objets archéologiques entre les blocs. S'agissait-il d'objets infiltrés dans des fissures ou appartenaient-ils à des couches intactes?

Le démontage de l'effondrement s'est fait principalement en forant une série de trous dans la roche et en utilisant des coins-éclateurs afin de les briser sans endommager le contexte sédimentaire environnant. Plusieurs de ces blocs mesuraient un mètre de largeur et étaient assez épais pour nécessiter de les éclater en suivant leur litage naturel. Quand nous avons enlevé une partie d'un grand bloc plat dans le niveau 5A (et dans le niveau 6A), nous avons découvert une couche très mince ( 1 à $2 \mathrm{~cm}$ ) de sédiments avec des objets archéologiques pris en étau entre le bloc enlevé et le bloc sous-jacent (fig. 39). Notre interprétation initiale était que ces objets, os et matériaux lithiques, devaient avoir été amenés par l'action de l'eau, après la chute des blocs. Deux arguments suggèrent que cela n'a pas été le cas. D'abord, si l'eau avait effectivement déplacé les objets, nous devrions trouver de nombreux objets de petite taille. Or, l'absence claire de petits objets contraste avec les objets dégagés dans la couche 5A. En second lieu, et peut-être de façon plus convaincante, dans chacune de ces deux surfaces d'occupation, nous avons trouvé un outil lithique éclaté en plusieurs morceaux (fig. 40). Apparemment, ces objets reposaient sur une grande dalle issue de l'effondrement du toit lorsqu'une nouvelle section s'est effondrée sur eux.
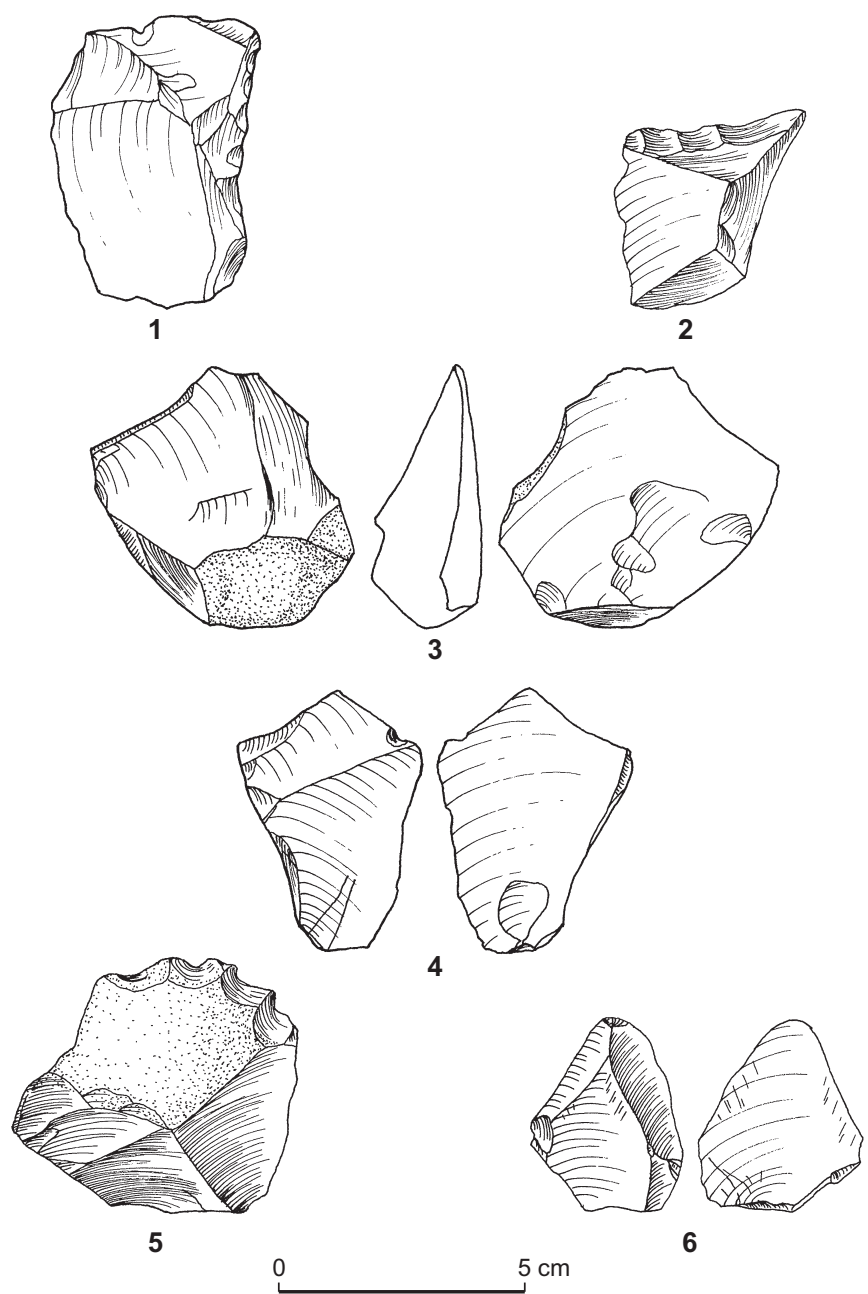

Fig. 32 - Couche 3A: 1, couteau à dos abattu typique; 2, éclat; 3, burin typique; 4, pointe pseudo-Levallois; 5, denticulé; 6, pointe Levallois (dessin: T. Tsenova).

La poursuite du dégagement des blocs nous a permis de mettre au jour un autre niveau identique au sein de la couche 6A: l'occupation 6A1. Ainsi, il semble qu'il y ait eu dans cette partie du site au moins trois épisodes d'effondrement du toit de la grotte. Une fois qu'une première partie est tombée, l'occupation de la grotte s'est poursuivie sur et derrière ces premiers blocs, jusqu'à ce que le toit s'effondre de nouveau. Contrairement à la partie nord du site, ces blocs d'effondrement, dans les carrés G12-H14, ont rendu toute sédimentation impossible et ont scellé, et ainsi protégé ces couches (niveaux 5A1 et 6A1). Nous ne pouvons pas estimer le temps représenté par chacune de ces couches, mais elles demeurent la structure archéologique la plus proche d'un «sol d'habitation" que nous pouvons attendre sur un site tel 

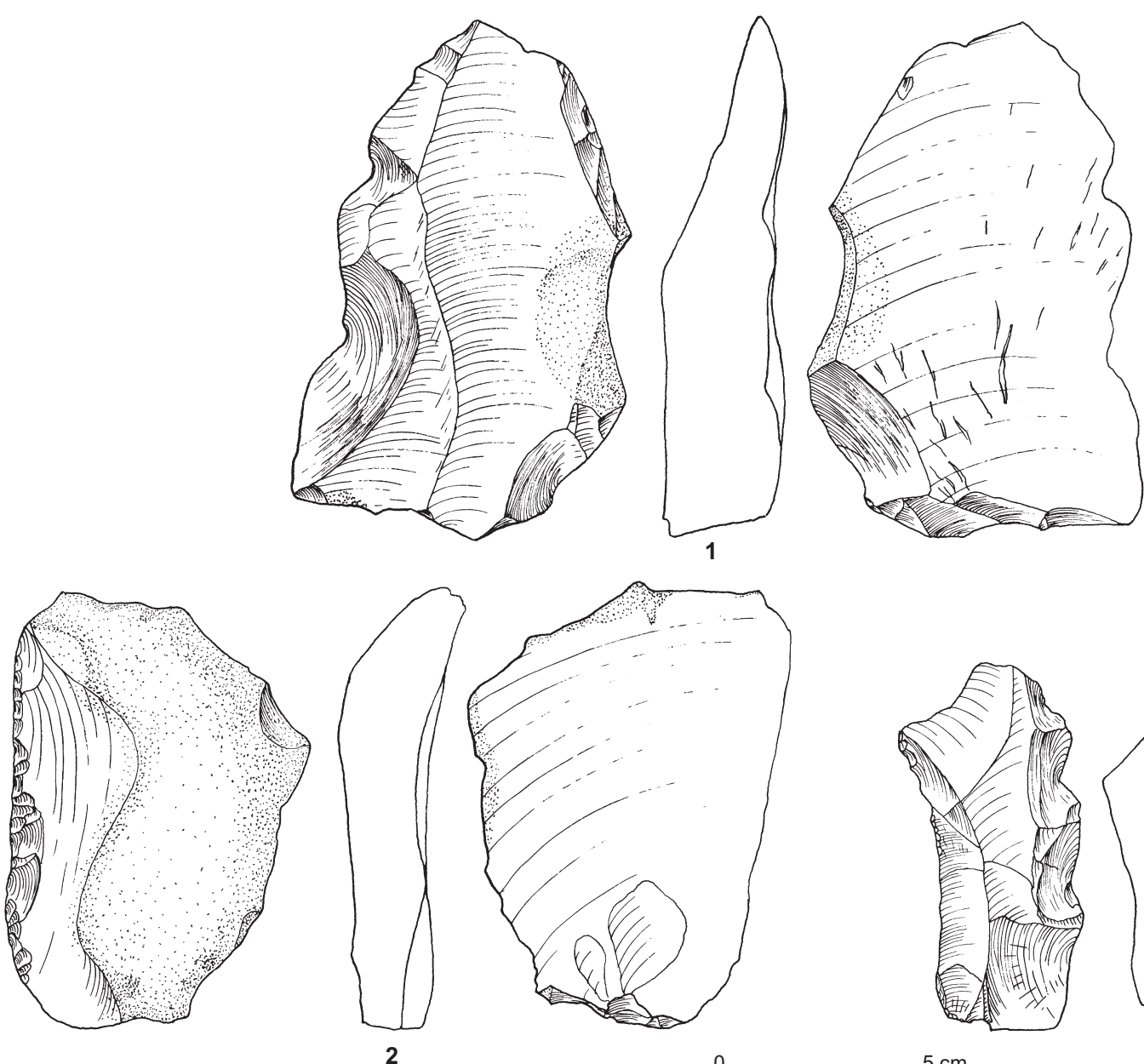

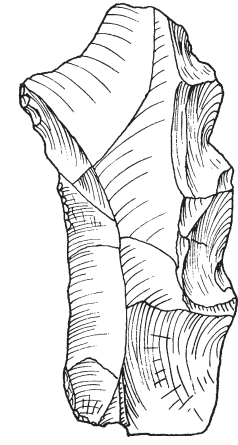

$5 \mathrm{~cm}$

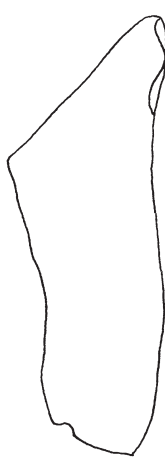

3

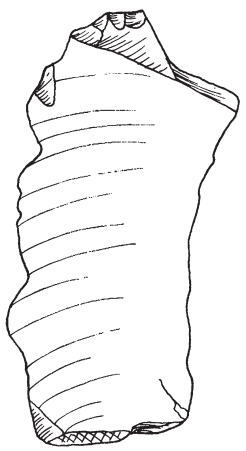

Fig. 33 - Couche 3A: 1, encoche; 2, racloir simple droit; 3, denticulé (dessin: T. Tsenova).

que le Pech de l'Azé IV. En tant que telles, elles devraient constituer une comparaison intéressante avec le reste du site où il n'est possible de localiser des phases d'occupation qu'au niveau d'une couche géologique, ou parfois d'une lentille de matériel à l'intérieur d'une couche. Un premier inventaire du contenu de chacun de ces niveaux est détaillé dans le tableau XXI. Des analyses plus détaillées sont en cours.

\section{LES FOYERS DE LA COUGHE 8}

Les zones de combustion stratifiées visibles dans les couches $\mathrm{X}, \mathrm{Y}$ et $\mathrm{Z}$ sont une autre découverte significative (fig. 10). Dans sa note préliminaire, F. Bordes (1975) mentionne à plusieurs reprises l'existence de niveaux de combustion stratifiés. Ils se définissent comme des ensembles sédimentaires de couleur noirâtre dans lesquels il est possible d'identifier des sous-ensembles lités et superposés. Comme expliqué ci-dessus, ces niveaux ne semblent pas être remaniés et représentent probablement des moments anthropogéniques, interprétés en tant que foyers, d'extension toutefois limitée. Considérant l'importance documentaire de ces zones de combustion dans un contexte moustérien, il avait été décidé de n'entreprendre leur exposition minutieuse dans la zone de la banquette (carrés D12, D13, E12, E13, F12 et F13) qu'à partir du moment où il était matériellement possible de les dégager sur une large surface. Cette précaution a permis une exposition maximale ainsi qu'une lisibilité verticale et horizontale accrue de ces structures. 

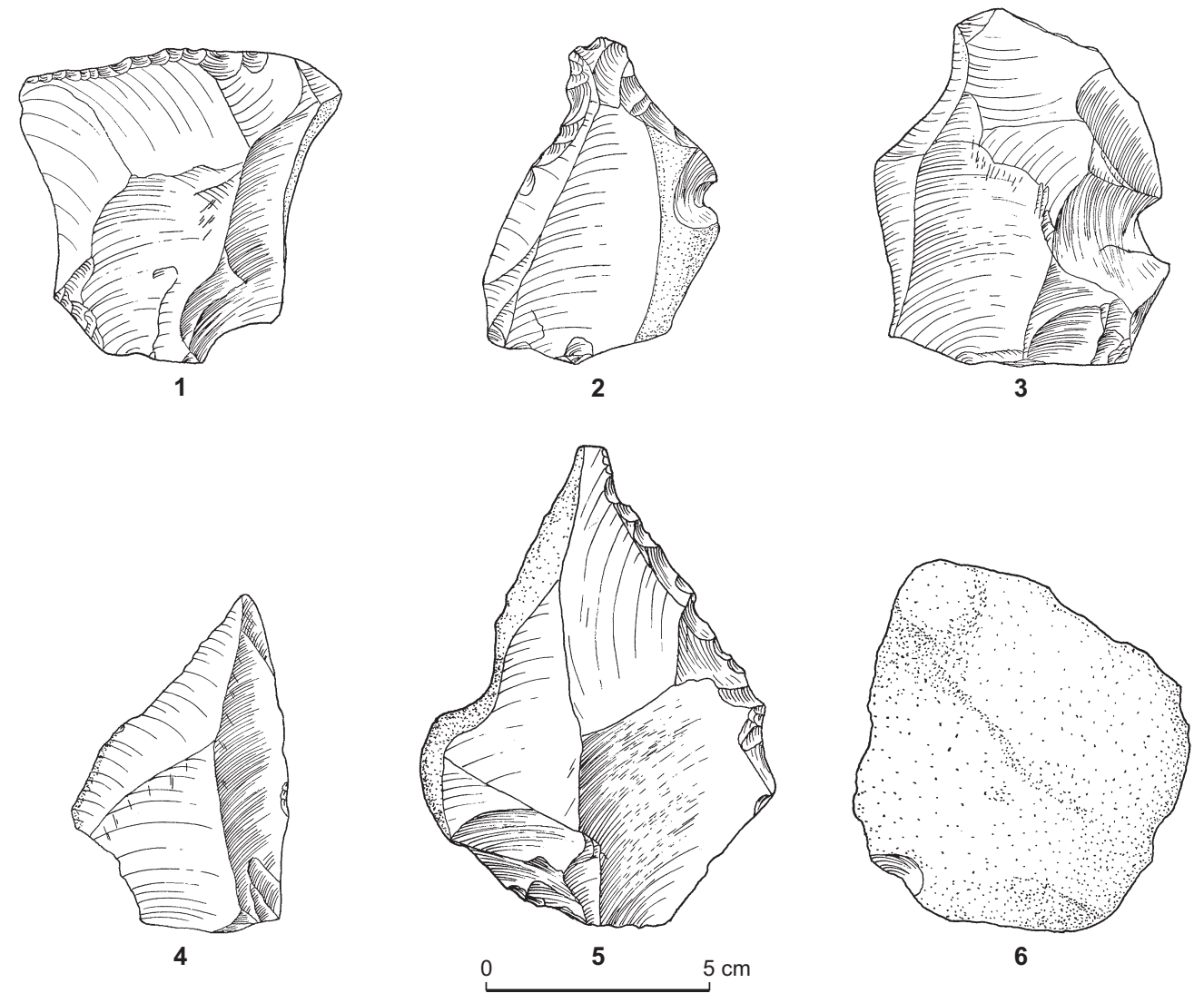

Fig. 34 - Couche 3B: 1, racloir transversal convexe; 2, couteau à dos abattu atypique; 3, éclat Levallois;

4, pointe Levallois; 5, denticulé; 6, éclat d'entame (dessin: T. Tsenova).
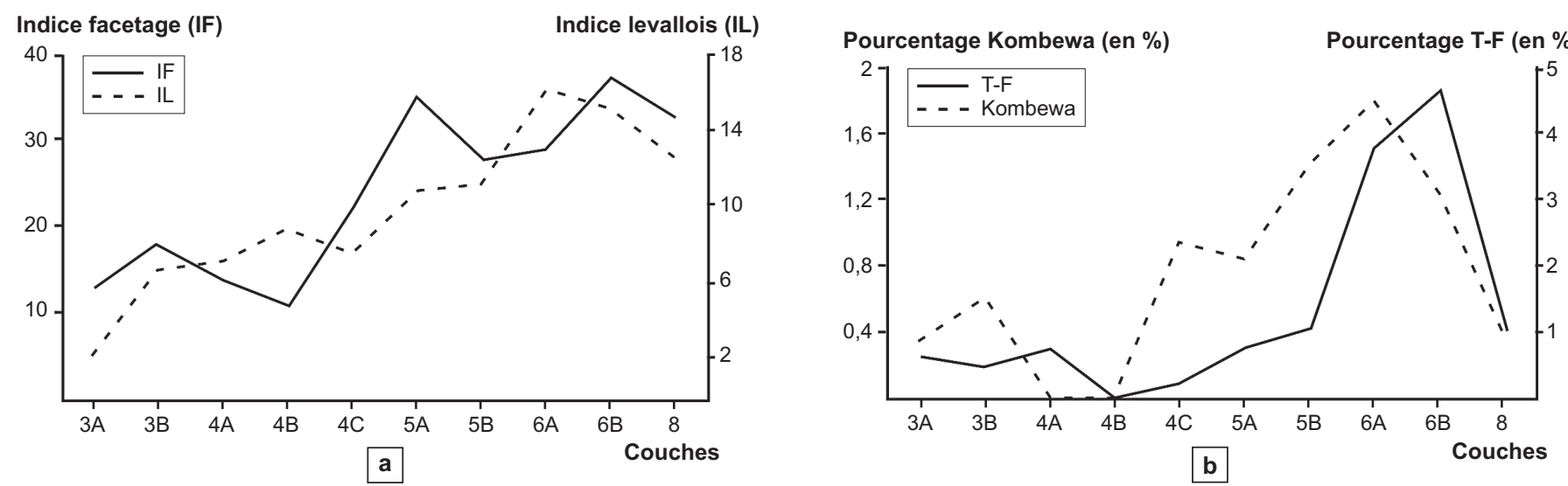

Fig. 35 - a, variation de l'indice de facetage et de l'indice Levallois dans la séquence; $\boldsymbol{b}$, variation du pourcentage d'éclats Kombewa et des pièces tronquées facettées dans la séquence (DAO: H. Dibble, Université de Pennsylvanie). 

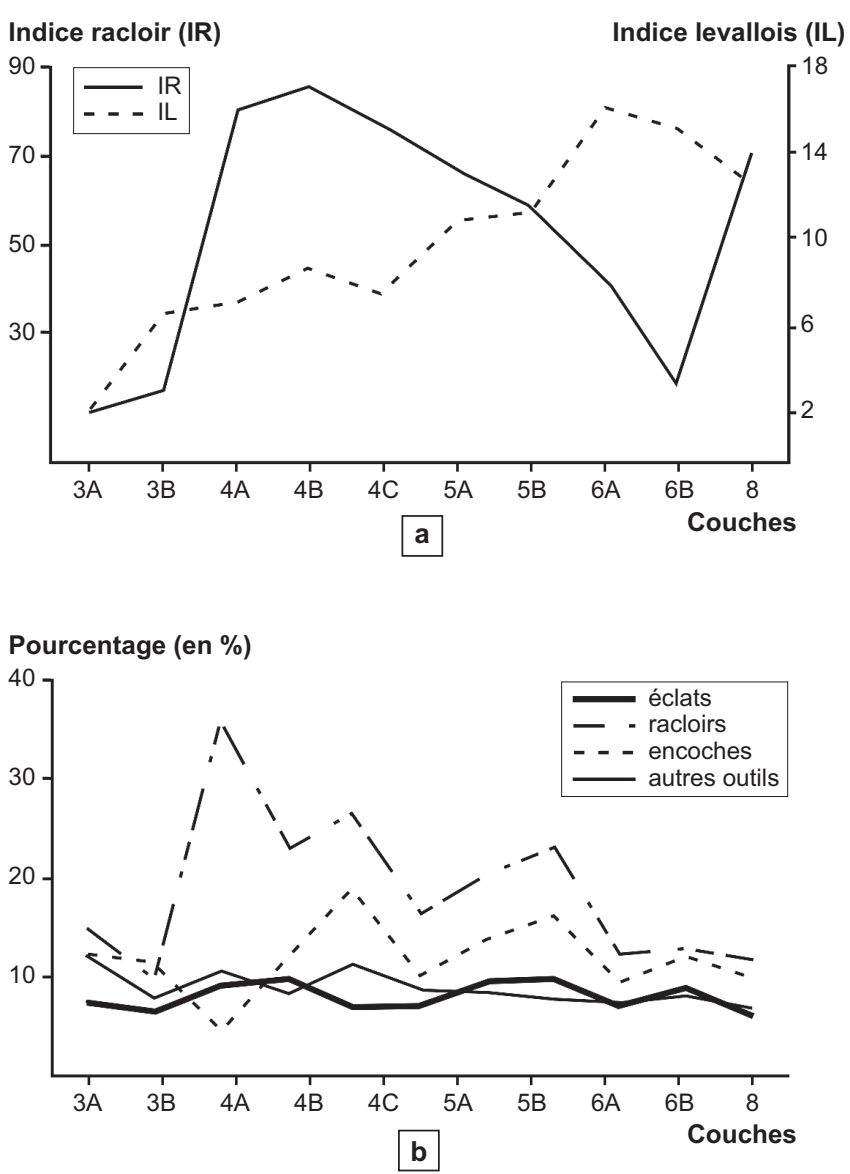

Fig. 36 - a, variation de l'indice de racloir et de l'indice Levallois; $\boldsymbol{b}$, variation du pourcentage de racloirs, encoches, autres outils et éclats dans la séquence (DAO: H. Dibble, Université de Pennsylvanie).

\section{MÉTHODOLOGIE}

En se basant sur les expériences acquises des membres de l'équipe dans des contextes archéologiques similaires, la mise en place d'une approche particulière des niveaux de base avait été décidée à la fin de la campagne 2001. Cette approche était motivée par la volonté d'appréhender ces structures de combustion avec le maximum de visibilité possible afin de pouvoir les identifier et les documenter de façon optimale. Dès lors, nous avons élaboré une méthodologie spécifique pour ces éventuelles aires de combustion en tenant compte des contraintes du gisement et du projet. Nous avons donc décidé d'isoler chacune des structures de combustion et de leur attribuer une numérotation et une abréviation qui vont être reprises dans le texte qui suit. Ainsi la structure de combustion 13 deviendra SC\#13.

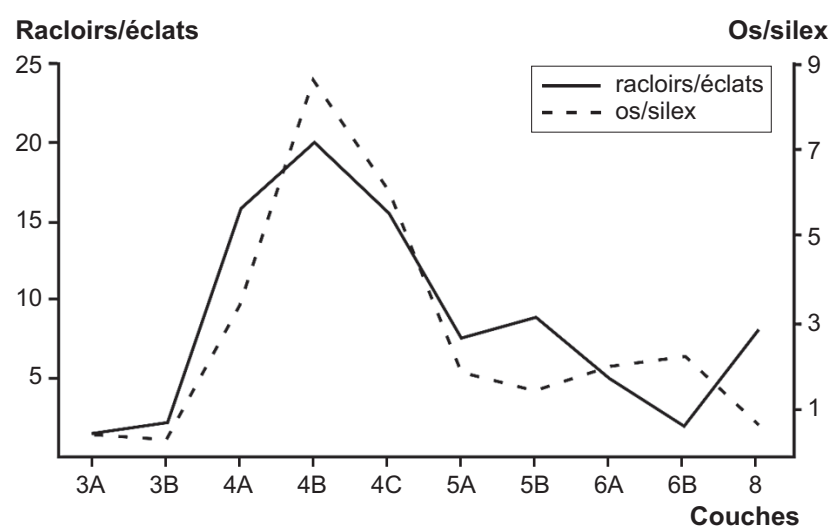

Fig. 37 - Variation du rapport racloirs/éclats et os/silex dans la séquence (DAO: H. Dibble, Université de Pennsylvanie).

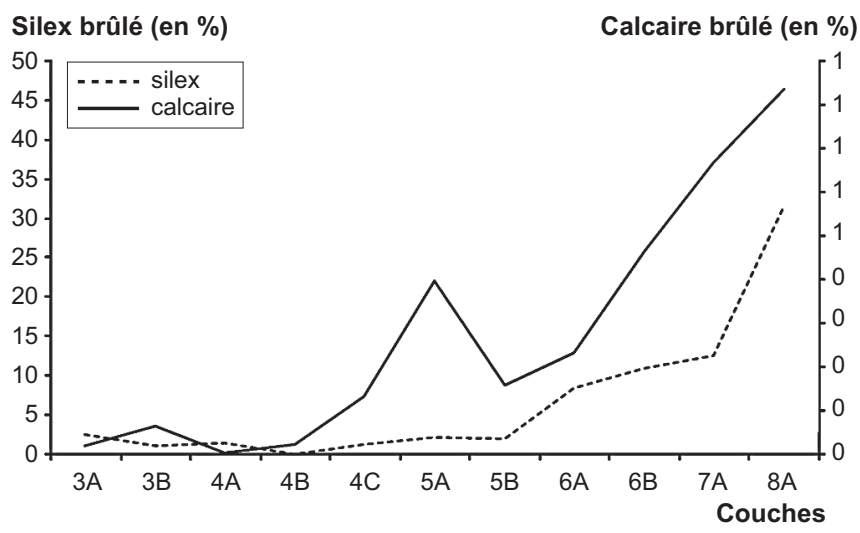

Fig. 38 - Variation du pourcentage de silex et de fragments de calcaire brûlés (DAO: H. Dibble, Université de Pennsylvanie).

Ces structures se définissent, lorsqu'elles sont le plus clairement visibles, par une zone isolée (par contraste avec la matrice) composée d'un sédiment argilo-sableux assez dense, d'aspect gras et de couleur noirâtre. Certaines de ces structures, surtout à la base de la couche 8-SC\#13, possédaient une composition comparable à celle identifiée pour certains foyers de la grotte XVI (Dordogne) avec une succession de sédiments sableux rouges à la base, d'une lentille noire et d'une zone orangée au sommet (Rigaud et al., 1995). En revanche, la majorité des structures au Pech de l'Azé IV sont plus désorganisées et cette succession n'existe que pour quelques-unes (SC\#7 et SC\#12). Celles-ci ressemblent davantage aux foyers décrits à Saint-Germaindes-Vaux (Manche) où des agglomérations cendreuses aux contours souvent flous ont été identifiées (Cliquet et al., 1989, p. 29). Même si la composition du sédiment varie 


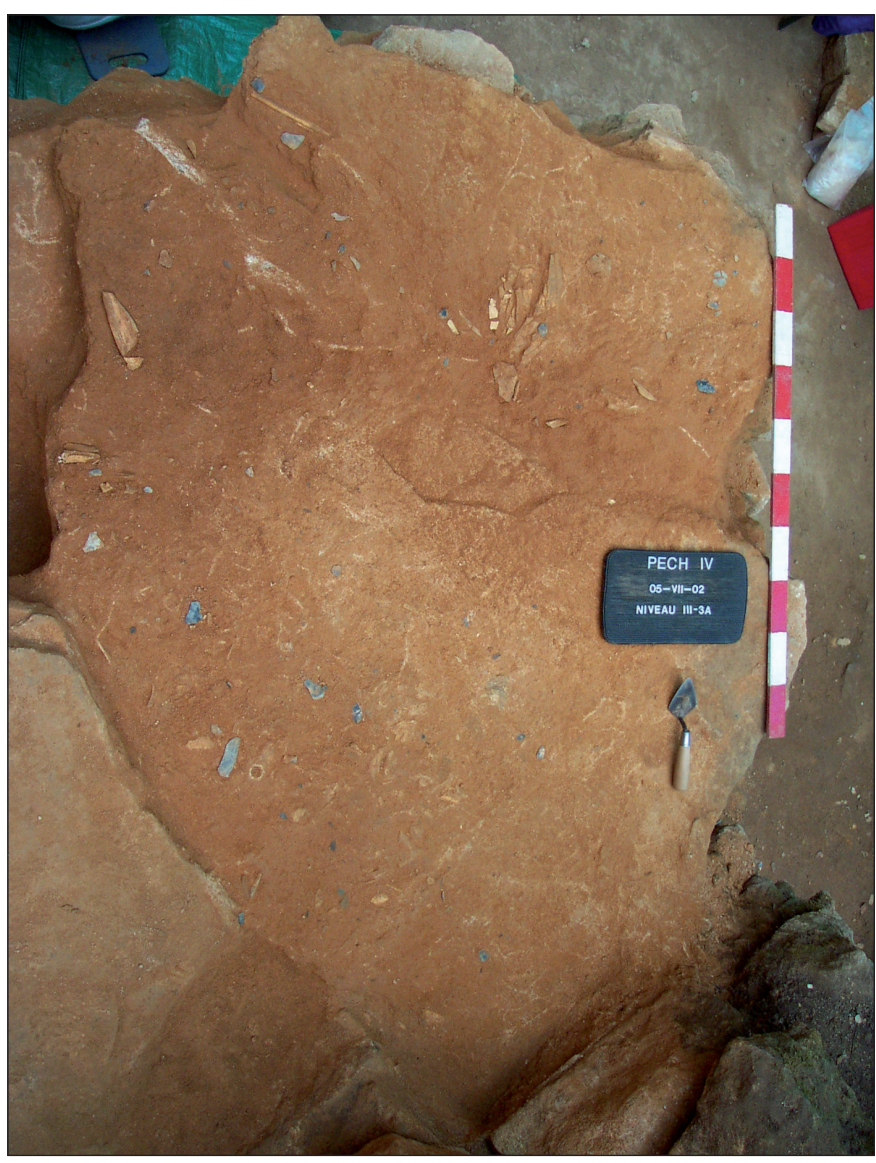

Fig. 39 - Niveau d'occupation 5A1 : surface après enlèvement des blocs d'effondrement (cliché: H. Dibble, Université de Pennsylvanie).

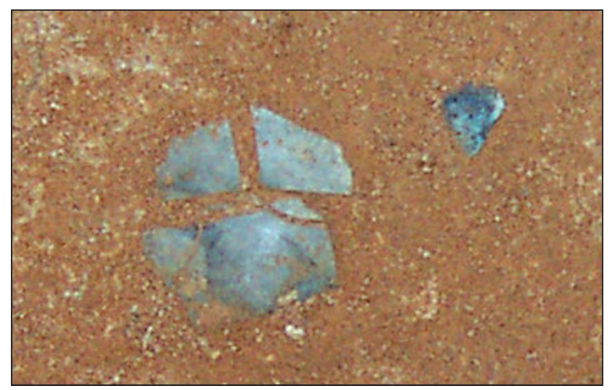

Fig. 40 - Niveau d'occupation 5A1: objet lithique cassé en place par blocs d'effondrement (cliché:

B. Maureille, Pacea, Université de Bordeaux-I).

quelque peu, elles contiennent pour la plupart des os brûlés et des objets lithiques, brûlés ou non, de taille assez variée. Elles sont le plus souvent de formes vaguement circulaires et mesurent une dizaine de centimètres de diamètre jusqu'à un mètre pour les plus grandes. L'interprétation fonction-
Tabl. XXI - a, contenu du niveau 5A-1; $\boldsymbol{b}$, contenu du niveau 6A-1.

\begin{tabular}{|c|c|c|c|}
\hline Couche & Type d'artéfact & Classe de vestige & Décompte \\
\hline \multirow{4}{*}{} & os & & 13 \\
\cline { 2 - 4 } & silex & & 3 \\
\cline { 2 - 4 } & silex & éclat entier & 3 \\
\cline { 2 - 4 } & silex & outil entier & 3 \\
\cline { 2 - 4 } & silex & fragment distal d'éclat & 2 \\
\cline { 2 - 4 } & silex & fragment mésial d'éclat & 1 \\
\cline { 2 - 4 } & silex & fragment proximal d'éclat & 1 \\
\cline { 2 - 4 } & silex & débris & 2 \\
\hline
\end{tabular}

a

\begin{tabular}{|c|c|c|c|}
\hline Couche & Type d'artéfact & Classe de vestige & Décompte \\
\hline \multirow{1}{*}{} & minéral & & 1 \\
\cline { 2 - 4 } & os & & 50 \\
\cline { 2 - 4 } & silex & & 3 \\
\cline { 2 - 4 } & silex & éclat entier & 10 \\
\cline { 2 - 4 } & silex & outil entier & 3 \\
\cline { 2 - 4 } & silex & nucléus & 1 \\
\cline { 2 - 4 } & silex & fragment de nucléus & 3 \\
\cline { 2 - 4 } & silex & fragment distal d'éclat & 9 \\
\cline { 2 - 4 } & silex & fragment distal d'outil & 1 \\
\cline { 2 - 4 } & silex & fragment mésial d'éclat & 2 \\
\cline { 2 - 4 } & silex & fragment mésial d'outil & 1 \\
\cline { 2 - 4 } & silex & minéral & 1 \\
\cline { 2 - 4 } & silex & fragment proximal d'éclat & 8 \\
\cline { 2 - 4 } & silex & débris & 4 \\
\cline { 2 - 4 } & silex & non taillé & 1 \\
\hline
\end{tabular}

b

nelle de ces structures reste problématique et il n'est pas possible, dans l'état de la fouille, d'avancer une quelconque hypothèse.

Pendant la campagne 2002, l'ensemble des carrés possédant des structures de combustion (carrés D12-F13) ont été dégagés en utilisant la méthode de décapage horizontal. Celle-ci consiste à dégager une surface, plus ou moins étendue, en suivant un niveau déterminé. Elle permet ainsi d'évaluer l'extension spatiale d'éventuelles structures archéologiques de façon minutieuse, et d'adapter les méthodes et techniques de fouille en fonction. Par sa visibilité horizontale et son adaptabilité, le choix de cette méthode a permis d'identifier des ensembles sédimentaires et de possibles aires de combustion. Nous avons limité le nombre de surfaces de décapage ouvertes à un même moment de la fouille. Si elle offre une grande visibilité horizontale, cette méthode doit être nécessairement complétée par un contrôle de la dimension verticale de la fouille. Sans cela, il reste souvent difficile d'identifier des structures ou de suivre leur progression. En ce sens, nous avons limité dès 
que possible les surfaces de décapage à des sous-carrés de $50 \mathrm{~cm}$ sur $50 \mathrm{~cm}$ ou moins, selon un schéma en damier pour l'ensemble de la banquette inférieure (carrés D12-F13).

Il nous a fallu en outre développer une méthode particulière pour identifier et nommer les éventuelles structures de combustion. En 2001, les informations accessibles étaient limitées à la description que F. Bordes avait faite des niveaux X, Y et Z (Bordes, 1975), aux analyses micromorphologiques préliminaires effectuées par P. Goldberg et aux coupes stratigraphiques révélées après l'abattement des murs de protection ${ }^{9}$. En se basant sur ces données, nous savions que nous aurions à identifier des structures de combustion à l'intérieur d'une matrice sablo-argileuse de couleur sombre à très sombre. Dès lors, l'enjeu était de pouvoir identifier de façon objective de subtiles structures archéologiques noyées dans une matrice de couleur similaire. Notre connaissance de structures approchantes observées en contexte moustérien nous a permis de formuler un cahier des charges assez précis. En revanche, les rares exemples de structures de combustion dans d'autres sites moustériens ne correspondaient pas aux conditions exactes que nous avions au Pech de l'Azé IV. Ainsi, il restait difficile de différencier de façon nette et définitive des zones sédimentaires au Pech de l'Azé IV.

Nous avons tenté différentes techniques pour enregistrer les coordonnées des foyers. L'utilisation du théodolite laser ne permettait pas de changer les coordonnées des limites des foyers au fur et à mesure du décapage de façon souple et aisée. Nous avons donc opté pour un compromis entre relevé topographique et utilisation de la station laser. Des relevés topographiques des différentes zones sédimentaires, et donc des structures de combustion, ont été réalisés à intervalles réguliers afin de rendre compte de leur évolution aussi bien horizontale (en plan) que verticale.

Ainsi, durant le décapage de chaque sous-carré, un relevé de la surface a été effectué toutes les deux heures. Ces plans, ou schémas topographiques, contiennent les coordonnées de toutes les zones sédimentaires identifiées et obtenues à partir du théodolite laser et une série de données décrivant les changements observés par le(s) fouilleur(s).

Les relevés de toutes les nouvelles coupes stratigraphiques ont été effectués sous la supervision du ou des fouilleur(s) concerné(s), du responsable de terrain et de P. Goldberg, afin de combiner toutes les observations fines

9. À la fin des fouilles de F. Bordes et pour protéger la base des coupes, des murs en béton avaient été construits. Leur destruction, lors de la campagne de fouille 2001, a nécessité plusieurs journées de travail. et de fournir un effort d'interprétation préliminaire des ensembles sédimentaires.

La documentation photographique réalisée tout au long des décapages et les photographies prises quotidiennement par le responsable de terrain ont permis de déterminer minutieusement des sous-zones dans les différentes couches.

La séparation de tous les sédiments issus de la structure a été faite ainsi que leur conditionnement dans des sacs à l'abri de la lumière pour réaliser une analyse anthracologique.

Les prélèvements micromorphologiques ont été effectués à plusieurs endroits de la zone de la banquette par P. Goldberg.

Chaque structure de combustion a été définie par son aspect (couleur, estimation à l'œil nu du sédiment, granulométrie, etc.) Ainsi, il y a création dès lors qu'un seul de ces critères varie de façon significative. Si cette méthode reste pour le moins subjective, elle permet un certain niveau de prudence et de la souplesse. En séparant chaque zone de la sorte, il nous sera possible de proposer, après un examen plus attentif de l'ensemble des données et plus particulièrement des données micromorphologiques, des hypothèses de corrélation.

\section{RÉSULTATS}

Un ensemble de 16 structures de combustion a été mis au jour (tabl. XXII). La majorité a été identifiée dans la partie sud de la banquette et les plus développées dans l'extrémité sud. La partie nord était en effet constituée d'un ensemble concrétionné qui a oblitéré les possibles structures de combustion s'y trouvant. Une seule, SC\#3, a été identifiée au sommet de la couche 8.

Trois types (sommet, intermédiaire et base) ont été créés pour donner une idée plus concrète de la localisation des différentes structures de combustion à l'intérieur de la couche 8 . Les coupes stratigraphiques tendent à définir trois moments assez distincts qui correspondent dans une certaine mesure aux trois niveaux (X, Y et $\mathrm{Z}$ ) définis par F. Bordes. Cependant, si on les considère de façon individuelle, il n'est pas possible d'identifier une organisation aussi claire. En effet, il semble que les différentes structures de combustion se superposent et se chevauchent tout au long de la séquence de manière similaire aux structures identifiées à la grotte de Kébara (Meignen et al., 1989, p. 144; Meignen et al., 2007). Cela explique partiellement notre difficulté à identifier les structures de façon précise et de suivre leur progression aussi bien horizontale que 
Tabl. XXII - Description des structures de combustion observées sur le terrain.

\begin{tabular}{|c|c|c|c|c|}
\hline Numéro & $\begin{array}{l}\text { Position } \\
\text { en coupe }\end{array}$ & $\begin{array}{c}\text { Extension horizontale approximative } \\
\text { (nord/sud, est/ouest) }\end{array}$ & Carré & Commentaire/Hypothèse \\
\hline 1 & sommet & $50 \mathrm{~cm}$ par $70 \mathrm{~cm}$ & F13/E13 & \\
\hline 2 & sommet & jusqu'à $150 \mathrm{~cm}$ de longueur & F12 /F13 & tronquée par coupe sud; extension certaine vers le sud \\
\hline 3 & sommet & $40 \mathrm{~cm}$ par $30 \mathrm{~cm}$ & $\mathrm{D} 12$ & dans zone concrétionnée avec remontée du bedrock \\
\hline 4 & intermédiaire & $20 \mathrm{~cm}$ par $20 \mathrm{~cm}$ & F13 & \\
\hline 5 & intermédiaire & non cartographiée & E13 & \\
\hline 6 & sommet & $20 \mathrm{~cm}$ par $10 \mathrm{~cm}$ & F13 & possible extension latérale de $\mathrm{BF} \# 2$ \\
\hline 7 & base & $20 \mathrm{~cm}$ par $40 \mathrm{~cm}$ & F13 & tronquée par coupe sud \\
\hline 8 & sommet & $60 \mathrm{~cm}$ par $25 \mathrm{~cm}$ & E12 & \\
\hline 9 & sommet & non cartographiée & E13 & \\
\hline 10 & sommet & $5 \mathrm{~cm}$ par $10 \mathrm{~cm}$ & F12 & \\
\hline 11 & sommet & non cartographiée & E13 & \\
\hline 12 & base & $90 \mathrm{~cm}$ par $40 \mathrm{~cm}$ & F12 & possible corrélation avec BF\# 13 ; tronquée par coupe sud \\
\hline 13 & base & $50 \mathrm{~cm}$ par $60 \mathrm{~cm}$ & $\mathrm{~F} 12$ & $\begin{array}{l}\text { possible extension latérale de BF\# } 12 \text {; tronquée par coupe sud et coupe ouest ; avec } \\
\text { sable rougi entre plaquettes du bedrock à sa base ; plaquettes du bedrock rubéfiées }\end{array}$ \\
\hline 14 & moyenne & $50 \mathrm{~cm}$ par $30 \mathrm{~cm}$ & F12/E12 & avec sable rougi à sa base \\
\hline 15 & intermédiaire & $10 \mathrm{~cm}$ par $10 \mathrm{~cm}$ & $\mathrm{E} 12$ & très localisée \\
\hline 16 & intermédiaire & $40 \mathrm{~cm}$ par $10 \mathrm{~cm}$ & & tronquée par coupe ouest \\
\hline
\end{tabular}

verticale. De plus, les décapages successifs ont révélé que la partie nord de la couche 8 au niveau de la banquette se composait d'une large zone concrétionnée et indurée s'élargissant même vers la paroi. Ce fort concrétionnement a totalement oblitéré les structures de combustion dans cette partie nord à l'exception de SC\# 3. L'extension horizontale des structures dans la partie sud de la banquette semble clairement indiquer que des structures de combustion ont probablement existé vers la paroi. Malheureusement, il semble qu'elles ont été complètement tronquées et effacées par le concrétionnement. En revanche, en se basant sur les observations faites à partir de la coupe ouest dans l'entrée du site ainsi que la nouvelle coupe sud, les structures de combustion paraissent non seulement plus développées mais encore plus organisées dans les carrés F12 et F13 ainsi que les carrés G14/13, H14/13, F14/13. Elles sont en effet bien mieux conservées dans cette partie sud où l'écoulement ou le ruissellement semblent ne pas avoir affecté autant l'intégrité de la couche 8 .

\section{RESTES HUMAINS}

Jusqu'à présent, le site du Pech de l'Azé IV n'était pas connu pour avoir livré des restes humains. Pourtant, deux vestiges ont mérité notre attention et permettent ainsi au site d'intégrer les gisements périgourdins dont la fouille a mis au jour des restes humains moustériens et qui apportent des connaissances nouvelles sur l'histoire du peuplement de cette région.

\section{UNE INCISIVE DÉCIDUALE ISSUE DES FOUILLES DE F. BORDES}

Le premier vestige correspond à une incisive centrale déciduale supérieure gauche (fig. 41). Elle a été mise au jour par F. Bordes et porte la mention P.A.IV, D12 1444, écrite sur la racine de la pièce.

Sur une petite étiquette abîmée accompagnant la dent, nous pouvons encore lire «P. Azé IV D12 1444 C F4». Il est très probable que $\mathrm{C} F 4$ nous renvoie à la couche $\mathrm{F} 4$, ce qui correspondrait, concernant la corrélation stratigraphique entre les fouilles F. Bordes et celles menées par la présente équipe, au niveau 3B (Dibble et al., 2005).

Cette dent est en excellent état de conservation. La couronne et la racine sont quasiment intactes. On peut noter une légère attrition du bord libre de la couronne (stade 2 selon Smith, 1984) qui est légèrement concave, ce qui nous assure que cette dent était fonctionnelle. L'apex est toujours ouvert, elle n'avait donc pas totalement achevé sa calcification racinaire correspondant au stade Rc (Moorrees et al., 1963). On peut supposer qu'elle a appartenu à un enfant de plus d'un an et de moins de deux ans, selon le schéma de la calcification et de l'éruption dentaire selon Ubelaker (1984). En effet, en dehors d'un traumatisme maxillo-facial très violent, une telle dent ne peut avoir été perdue pour des raisons naturelles et il est très probable que cet enfant soit décédé sur le site.

La face vestibulaire est convexe verticalement et horizontalement. Dans le second plan, cette courbure est très 


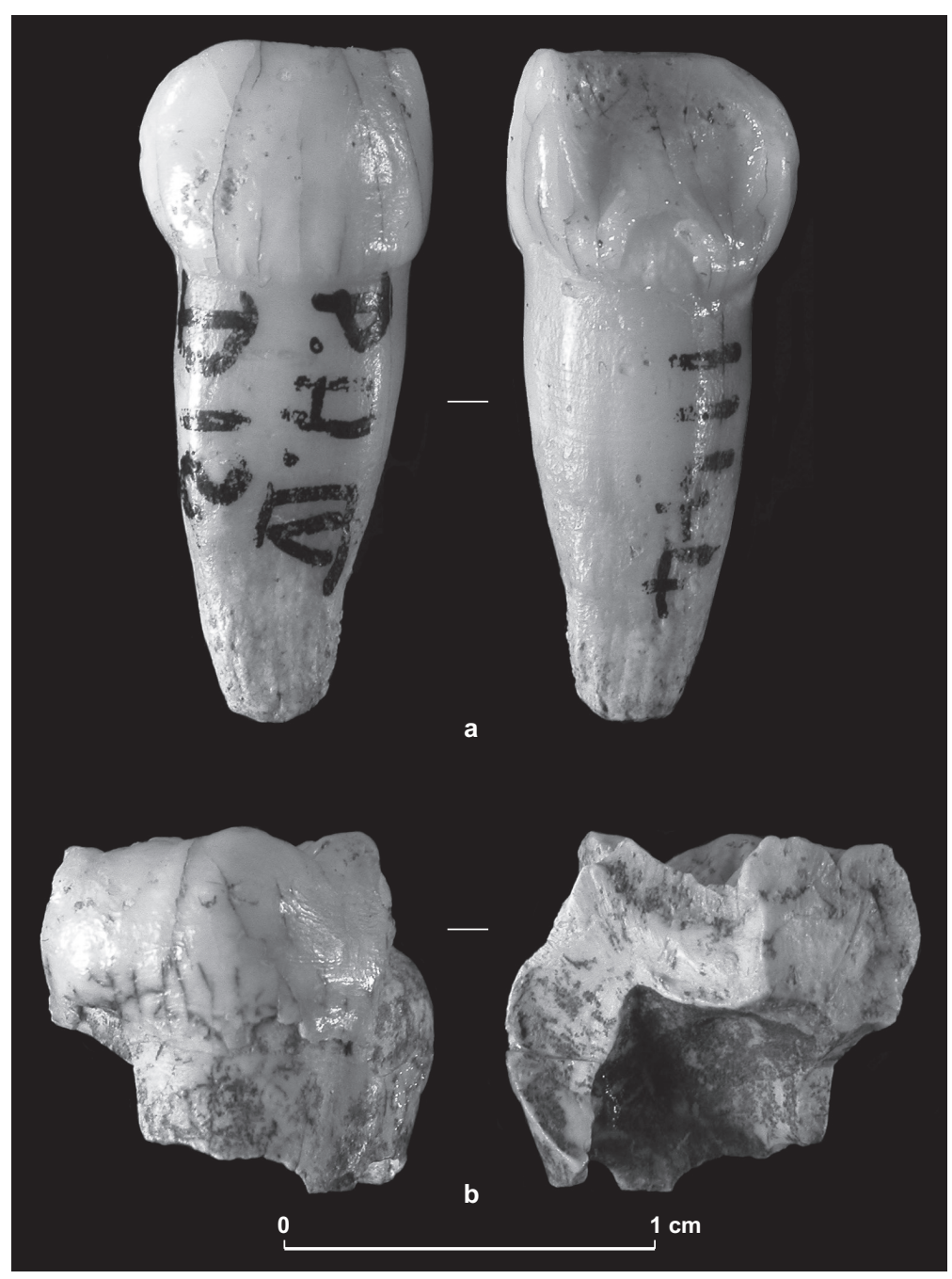

Fig. 41 - Une incisive déciduale découverte par F. Bordes et un fragment de molaire (cliché: B. Maureille, Pacea, Université de Bordeaux-I).

marquée à mi-hauteur de la couronne. La face distale l'est également dans un plan vertical et montre une petite facette interproximale de contact. La face mésiale est nettement moins courbe que la précédente et paraît donc plus verticale. Elle semble aussi moins haute et montre une facette interproximale de contact. La face linguale présente un net tuberculum lingual bien saillant. Il est arrondi, non divisé et un peu dévié mésialement. Un tout petit morceau d'émail manque au niveau le plus saillant de ce tubercule, la dent ayant été préalablement consolidée. Un relief triangulaire s'enracine sur ce tubercule et le prolonge vers le bord libre de la couronne. Il est nettement séparé du bord distal par un sillon, et l'est un peu moins nettement du bord mésial. Ces deux bords forment deux reliefs bien marqués qui dessinent une pelle très nette. Du côté mésial, le repli est moins marqué et saillant que du côté distal où il paraît plus épais. Par rapport à d'autres Néandertaliens, la dent de la grotte des Cèdres par exemple, la pelle est atténuée (Vandermeersch, 1995). Sur la dent $n^{\circ} 32$ de la grotte d'Arcy-sur-Cure (Bailey, Hublin, 2006), on ne peut apprécier ce trait alors que sur celles de l'enfant du Pech de l’Azé I (Legoux, 1970), la morphologie de la pelle est très proche de celle de la dent du Pech IV.

La racine est bien conservée. Elle est très large sous le collet et cette largeur diminue régulièrement vers l'apex. Sa section s'inscrit globalement dans une ellipse. Contrairement à l'incisive centrale néandertalienne de la grotte des Cèdres (Vandermeersch, 1995), en vue mésiale ou distale, 
l'angulation du tiers apicale de la racine vers la face vestibulaire est peu marquée. B. Vandermeersch avait suspecté un traumatisme pour expliquer la forte angulation de la racine. Il n'en est rien, cette angulation est un trait morphologique normal des incisives déciduales centrales qui existe souvent sur les dents actuelles. En revanche, le tiers apical de la dent paraît un peu tourmenté.

Nous n'avons pas encore réalisé de radiographie de la pièce, en revanche, elle a été microscannée au Max Plank Institut de Leipzig. Cela nous a permis d'observer directement la morphologie d'un canal radiculaire au niveau de la section de la racine et les proportions relatives des différents constituants de la dent (émail, dentine, pulpe par le volume de la chambre pulpaire). Sur cette incisive, on ne note aucune pathologie ni indicateur de stress non spécifique.

Selon nous, par l'ensemble de ses caractéristiques morphologiques, cette dent s'intègre mieux au sein de la variabilité néandertalienne que dans celle des hommes modernes du Paléolithique supérieur ou des hommes actuels.

En l'état, la dent mesure 17,95 mm de haut. Cette valeur n'a d'autre intérêt que de fournir un élément permettant d'apprécier la conservation du vestige dentaire dans le futur si des analyses isotopiques ou paléobiochimiques étaient réalisées à partir d'un prélèvement de racine. Le diamètre mésio-distal est de 7,70 $\mathrm{mm}$ et le diamètre vestibulo-lingual de 5,70 $\mathrm{mm}$. Nous pouvons considérer qu'aucun de ces diamètres n'est affecté par l'attrition dentaire, ils ne sont donc pas sous-estimés. Ils sont plus importants que la moyenne des enfants actuels et proches de la limite supérieure à $95 \%$ de la variabilité actuelle (tabl. XXIII). Par rapport à la variabilité des dents des Néandertaliens würmiens, on peut souligner que le diamètre mésio-distal de l'incisive de la grotte du Noisetier est légèrement supérieur à la moyenne et le diamètre vestibulo-lingual n'en est que légèrement inférieur. Ces deux diamètres sont logiquement inférieurs à la moyenne des incisives déciduales supérieures de la lignée néandertalienne. La dent fossile a donc des dimensions coronaires importantes relativement à la variabilité actuelle et moyennes relativement à celle des Néandertaliens du Moustérien récent.

L'indice coronaire de l'incisive de la grotte du Noisetier est de 74, alors que le module de robustesse (ou aire de la couronne) est au minimum de 43,89. La première valeur est très légèrement inférieure à la moyenne actuelle $(n=47$, moyenne $=77,6, \mathrm{~s}=7,98)$. Le second paramètre est nettement plus important que la limite supérieure à $95 \%$ $(39,4)$ ou à $99 \%(42,43)$ de la variabilité actuelle $(n=47$, moyenne $=30,26, \mathrm{~s}=4,53$ ). L'incisive déciduale de la grotte du Noisetier est donc plutôt volumineuse avec une longueur importante relativement à sa largeur. Ces traits métriques confirment son appartenance à la lignée néandertalienne.

Par ses caractéristiques morphologiques (Patte, 1955; Bailey, Hublin, 2006) et métriques (Maureille, 2001), il ne fait aucun doute que cette incisive déciduale s'intègre mieux au sein de la variabilité des dents néandertaliennes que des dents actuelles (Hillson, 2002; Lautrou, 1997).

À ce stade de l'étude, le NMI des sujets moustériens est donc de 1: un très jeune enfant de 1 à 2 ans provenant du niveau $3 \mathrm{~B}$.

\section{UN FRAGMENT DE GOURONNE DE MOLAIRE}

Cette pièce a été découverte le 29 juin 2002 durant les opérations de tamisage du sédiment fin provenant du seau 71 (fig. 42). Il correspondait à la fouille du carré F11 et du niveau 5A (Dibble et al., 2005). Le numéro de la pièce est 4490 .

Ce fragment de molaire est une petite partie d'une surface occlusale présentant une complexité importante des reliefs avec de nombreux sillons et des crêtes formant, pour certains, la partie d'un pan d'une cuspide. Outre le morceau de couronne, la partie attenante de la racine sur toute sa hauteur, est également conservée. La morphologie de

Tabl. XXIII - Effectif, moyenne, écart-type, limite supérieure et inférieure à $95 \%$ de la variabilité de la population estimée chez les hommes actuels, les Néandertaliens würmiens et la lignée néandertalienne quant au diamètre mésiodistal ou longueur de la couronne (MD) et au diamètre vestibulo-lingual ou largeur de la couronne (VL) de la dent.

\begin{tabular}{|l|c|c|c|c|c|c|}
\cline { 3 - 7 } \multicolumn{2}{c|}{} & Effectif & Moyenne & Écart-type & Limite supérieure 95 \% & Limite inférieure 95 \% \\
\hline \multirow{2}{*}{ Hommes actuels } & $\mathrm{MD}$ & 335 & 6,5 & 0,406 & 7,34 & 5,74 \\
\cline { 2 - 7 } & $\mathrm{VL}$ & 337 & 5,0 & 0,417 & 5,88 & 4,24 \\
\hline \multirow{2}{*}{$\begin{array}{l}\text { Néandertaliens } \\
\text { Würmiens }\end{array}$} & $\mathrm{MD}$ & 21 & 7,59 & 0,5 & 8,64 & 6,54 \\
\cline { 2 - 7 } & $\mathrm{VL}$ & 21 & 5,95 & 0,26 & 6,49 & 5,42 \\
\hline \multirow{2}{*}{$\begin{array}{l}\text { Lignée } \\
\text { néandertalienne }\end{array}$} & $\mathrm{MD}$ & 25 & 7,69 & 0,54 & 8,80 & 6,57 \\
\cline { 2 - 7 } & $\mathrm{VL}$ & 25 & 6,08 & 0,38 & 6,88 & 5,29 \\
\hline
\end{tabular}




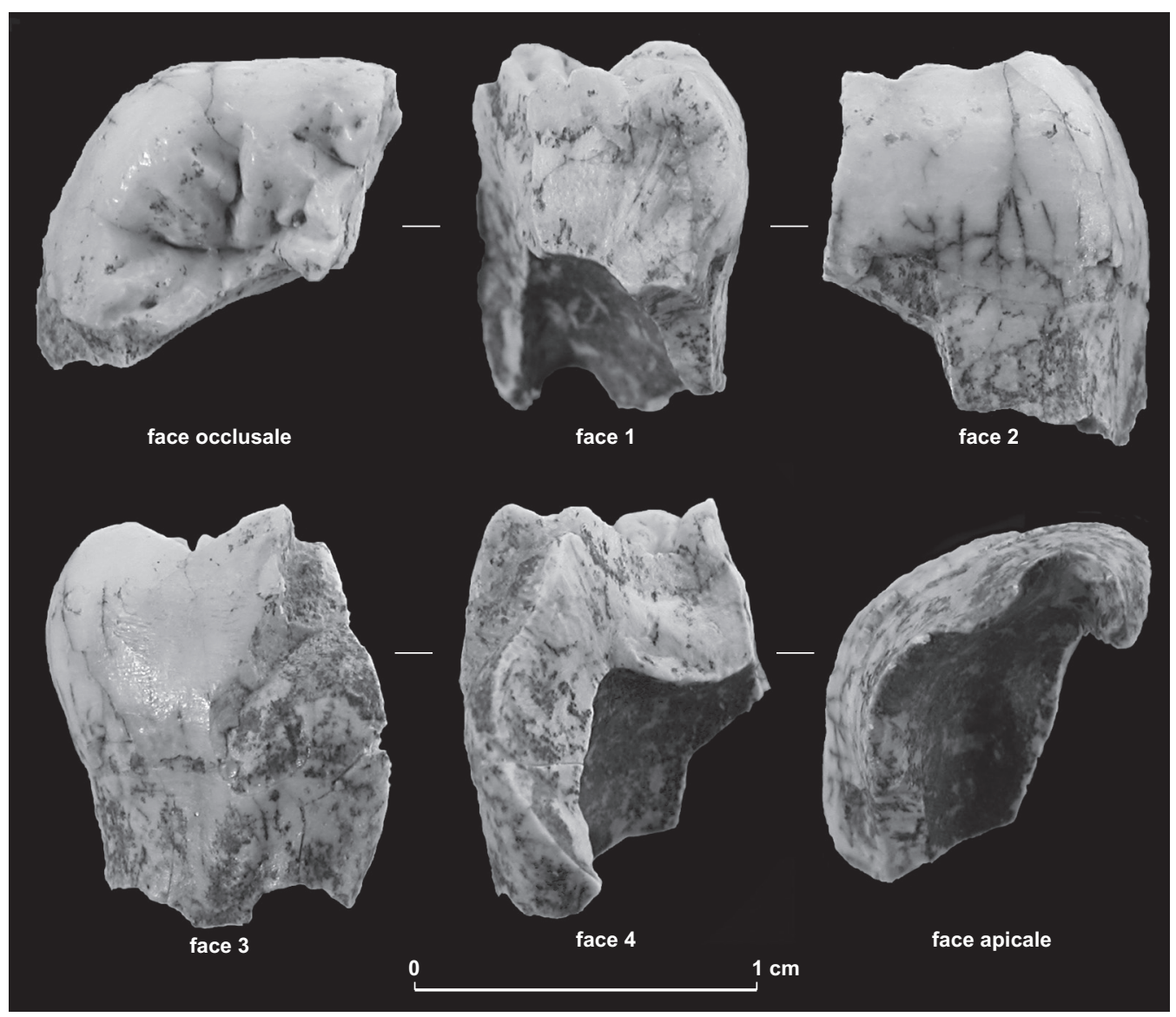

Fig. 42 - Un fragment de couronne de molaire (cliché: B. Maureille, Pacea, Université de Bordeaux-I).

cette dernière et de son bord libre nous assurent qu'il s'agit d'un morceau de germe de molaire. La racine est donc calcifiée sur 2,5 mm de hauteur (ou stade E, Demirjian et al., 1973). On ne note pas de corne déprimant le plafond de la chambre pulpaire. Cette morphologie orienterait plutôt la diagnose de ce fragment vers une dent supérieure. Dans ce cas, en fonction du développement en hauteur de la cuspide, de la courbure du bord libre, il pourrait correspondre à la cuspide disto-lingual (ou hypocône) d'une molaire supérieure droite. En fonction du développement de la racine, le degré de calcification varierait de 4 à 5 ans pour une première molaire, 8 à 9 ans pour une seconde molaire et au moins 15 ans pour une troisième. Naturellement, cette dent n'a jamais été fonctionnelle.

On ne peut souligner aucun trait particulier dans la partie conservée de la face occlusale. L'hypocône reste assez bien individualisé. Cela plaiderait plutôt pour une première ou une seconde molaire. La comparaison avec des dents néandertaliennes peu usées nous a permis de souligner cer- taines similitudes de ce fragment avec la première molaire supérieure droite du Moustier 1.

Les parties conservées de la dent ne nous permettent pas de tirer des conclusions quant à sa variabilité métrique. Notons toutefois, au niveau de sections, une dentine assez épaisse sous un émail mesurant presque $1 \mathrm{~mm}$ de haut au niveau des reliefs des crêtes.

Le site du Pech de l'Azé IV a livré à chaque campagne de fouilles un reste dentaire. Le premier est une incisive déciduale centrale supérieure d'un enfant de 1 à 2 ans. Le second est un fragment de couronne de germe de molaire supérieure peut-être plus probablement une première molaire ou une seconde qu'une troisième. Elle pourrait appartenir à un enfant de 4 à 5 ans (première hypothèse) ou de 8 à 9 ans (seconde hypothèse).

L'incisive par ses traits présente toutes les caractéristiques d'une dent néandertalienne. La morphologie du fragment de molaire ne permet pas de préciser la diagnose taxinomique du spécimen. On peut toutefois souligner ses 
similitudes avec la première molaire supérieure droite de l'adolescent du Moustier 1.

Les deux vestiges provenant de niveaux différents, le NMI est donc de 2 dans ce gisement. Aucune de ces dents ne montre d'atteintes pathologiques et ne peuvent avoir été perdues naturellement.

\section{CONCLUSIONS ET PERSPECTIVES}

Les nouvelles fouilles au Pech de l'Azé IV et les premières analyses ont apporté des informations inédites et importantes, sans toutefois bouleverser les données publiées par F. Bordes.

La nature même de la structure d'accueil a été précisée. Le site était une grotte plutôt qu'un abri sous roche et au moment de l'occupation, il ressemblait beaucoup au Pech de l'Azé I. La nouvelle lecture stratigraphique a confirmé dans les grandes lignes les observations faites par F. Bordes, mais les méthodes utilisées ont permis de préciser plusieurs points: la formation du site avec la mise en évidence des lacunes stratigraphiques et des phénomènes d'érosion et de ruissellement. L'étude des processus postdépositionnels, prenant en compte les données issues de la géologie et de l'archéologie, montre que les dépôts étaient globalement très bien conservés avec peu ou pas de modifications.

Pour ce qui est de l'âge des dépôts, les dates ESR placent notre niveau 3B (F4 de F. Bordes) approximativement entre $49 \mathrm{ka}$ et $56 \mathrm{ka}$ et la base (couche 8) vers $99 \mathrm{ka}$, ce qui va dans le même sens que les propositions faites antérieurement.

Les dépôts de la base de la séquence, particulièrement remarquables, ont livré plusieurs exemples d'utilisation du feu par les Néandertaliens.

Par ailleurs, les données géologiques et archéologiques (analyse détaillée des diagrammes de projection, composition des séries, altération) nous autorisent à proposer une corrélation des deux séquences et à dire que les études des séries lithiques rassemblées lors des récentes fouilles sont très comparables à celles issues des travaux de F. Bordes. Cela montre que notre nouvelle stratigraphie correspond à la sienne, ce qui revient à dire que:

- les deux collections peuvent être étudiées ensemble dans leur totalité;

- nous avons maintenant une meilleure connaissance de l'Asinipodien, une industrie lithique originale définie pour la première fois au Pech de l'Azé IV, qui démontre la volonté de produire de petits éclats au Paléolithique moyen;

- dans le domaine de la stratigraphie chronoculturelle du site, les fouilles ont révélé la présence de Moustérien de type Quina dans la couche 4 en une position habituelle dans la région, à savoir au-dessus d'ensembles Levallois et en dessous des ensembles à débitage mixte Levallois-discoïde.

De nouveaux et nombreux travaux sur les collections lithiques, fauniques et sur les dates numériques sont encore en cours. Le croisement de l'ensemble des données permettra de mieux comprendre les différentes occupations du site et plus généralement les comportements des Néandertaliens.

$$
\text { * } *
$$

Nous remercions les fouilleurs Taryn Ortoli, Nancy Odegaard, l'équipe de l'Arizona State Museum pour nous avoir donné accès au FTIR, les différents organismes et fondations qui ont contribué au financement de l'opération: National Science Foundation, Leakey Foundation, l'University of Pennsylvania, Museum of Archaeology and Anthropology, l'University of Pennsylvania Research Foundation, le Service régional de l'archéologie d'Aquitaine et le Conseil général de la Dordogne. Une mention particulière va à Tsenka Tsenova qui a réalisé l'ensemble des dessins du matériel lithique, à M. Lenoir et à D. de SonnevilleBordes qui nous a confié l'incisive qui «dormait» dans les collections et les archives de F. Bordes. 


\title{
BIBLIOGRAPHIE
}

\author{
ABRÉVIATIONS \\ APRAIF Association pour la recherche archéologique en Île-de-France. \\ BSPF Bulletin de la Société préhistorique française. \\ CNRS Centre national de recherche scientifique. \\ SPF Société préhistorique française.
}

\section{ANONYME}

1908: "Grottes du Pey-de-l'Ase et de CombeGrenal: lettres de l'abbé Audierne à de Mourcin", Bulletin de la société historique et archéologique du Périgord, 35, p. 121-131.

\section{AUDIERNE A.}

1863: De l'origine et de l'enfance des arts en Périgord: de lâge de la Pierre dans cette province avant la découverte des métaux, Périgueux, Imprimerie Dupont et Cie, 56 p.

BAILEY S., HUBLIN J.-J.

2006: "Dental Remains from the Grotte du Renne at Arcy-sur-Cure (Yonne) ", Journal of Human Evolution, 50, 5, p. 485-508.

BENN D. I.

1994: "Fabric Shape and the Interpretation of Sedimentary Fabric Data", Journal of Sedimentary Research, 64, p. 910-915.

\section{Bertran P., Hetu B., TeXier J.-P.,} VAN STEIN H.

1997: «Fabric Characteristics of Subaerial Slope Deposits », Sedimentology, 44, p. 1-16.

\section{BERTRAN P., TEXIER J.-P.}

1995: «Fabric Analysis: Application to Palaeolithic Sites ", Journal of Archeological Sciences, 22,4 , p. 521-535.

\section{BORDES F}

1954: «Les gisements du Pech de l'Azé (Dordogne) -I- Le Moustérien de tradition acheuléenne ", L'Anthropologie, 58 5-6, p. 401-432.

1955: «Les gisements du Pech de l'Azé (Dordogne) : le Moustérien de tradition acheuléene (suite), avec une note paléontologique de J. Bouchud», L'Anthropologie, 59, $1-2$, p. 1-38.

1972: A Tale of Two Caves, New York, Harper and Row, $169 \mathrm{p}$.

1975: «Le gisement de Pech de l'Azé IV: note préliminaire », $B S P F, 72$, p. 293-308.

1978: "Typological Variability in the Mousterian Layers at Pech de l'Azé I, II and IV", Journal of Anthropological Research, 34, 2, p. 181-193.

1981: «Vingt-cinq ans après: le complexe moustérien revisité », $B S P F, 78,3$, p. 77-87.

\section{BORDES F., BOURGON M.}

1950: «Le gisement du Pech de l'Azé-Nord: prise de date et observations préliminaires », $B S P F, 47,6-8$, p. 381-383.

1951: «Le gisement du Pech de l'Azé-Nord, campagnes 1950-1951: les couches inférieures à Rhinoceros mercki ", $B S P F, 48$, $11-12$, p. $520-538$.

\section{BOURGON M.}

1957: Les Industries moustériennes et prémoustériennes du Périgord, Paris, Masson (coll. Archives de l'Institut de paléontologie humaine, 27), $142 \mathrm{p}$.

Bourguignon L., FAIVRe J.-P., TURQ A.

2004: «Ramification des chaînes opératoires: une spécificité du Moustérien?", Paléo, 15 , p. $37-48$.

BOWMAN S., LOOSEMORE R., SIEVEKING G. DE G., BORDES F.

1982: «Preliminary Dates for Pech de l'Azé IV", Pact: revue du groupe européen d'études pour les techniques physiques, chimiques et mathématiques appliquées à l'archéologie, 6, p. 362-369.

BRAillard L., GUelat M., ReNTzel P.

2004: "Effects of Bears on Rockshelter Sediments at Tanay Sur-les-Creux, Southwestern Switzerland", Geoarchaeology, 19 4, p. 343-367.

BULlOCK P., FEDEROFF N., JONGERIUS A., STROOPS G., TURSINA T., BABEL U.

1985: Handbook for Soil Thin Section Description, Wolverhampton, Waine Research Publications, $152 \mathrm{p}$

\section{CAPDEVILLE J.-P.}

1987: Carte géologique de Sarlat (1/50 000) et notice, Orléans, BRGM, 28 p.

\section{CaPitan L., Peyrony D.}

1909: «Deux squelettes humains au milieu de foyers de l'époque moustérienne», Revue de l'école d'anthropologie, 19, p. 402-409.

\section{CHEYNier A.}

1936: Jouannet, grand-père de la Préhistoire, Brive, Imprimerie Chastrusse Praudel \& Cie, 102 p.

Cliquet D., DUMONT J.-L, DUPONT J.-P., FOSSE G., JOUIS M., VAN LIET-LANOE B., MANTE C., MOREL J., QUILlaRd J., THIÉBAUT S., VILGRAIN G.

1989: «Approche d'une étude comparative des matières organiques de foyers préhistoriques et de foyers expérimentaux: l'exemple du gisement moustérien de Saint-Germain-des-Vaux (Manche) ", in OLIVE M., TABORIN Y. (DIR.), Nature et fonction des foyers préhistoriques, Actes du colloque international de Nemours, 12-14 mai 1987, APRAIF (coll. Mémoires du musée de Préhistoire d'Île-de-France, 2), p. 29-36.

\section{DELPORTE H., DAVID F.}

1965: «L'évolution des industries moustériennes à la Rochette, commune de SaintLéon-sur-Vézère (Dordogne) », BSPF, 62 , 1, p. $48-62$

\section{DEMARS P.-Y.}

1982: L'Utilisation du silex au Paléolithique supérieur: choix, approvisionnement, circulation, l'exemple du bassin de Brive, Paris, CNRS Éditions (coll. Cahiers du Quaternaire, 5), $253 \mathrm{p}$.

\section{DEMIRJIAN A., GOLDSTEIN H.,} TANNER J. M.

1973: "A New System of Dental Age Assessment», Human Biology, 45, 2, p. 211-227.

DIBBLE H.

1987: «Direct Measurement of Artifact Provenience with an Electronic Theodolite", Journal of Field Archaeology, 14, p. 249-254. 
1988: «Typological Aspects of Reduction and Intensity of Utilization of Lithic Resources in the French Mousterian", in Dibble H., MonTET-White A. (DIR.), Upper Pleistocene Prehistory of Western Eurasia, Philadelphie, University Museum Monograph, 54, University Museum Symposium Series, p. 181-197.

1991: «Local Raw Material Exploitation and its Effects on Lower and Middle Paleolithic Assemblage Variability», in Montet-White A., Holen S. (DIR.), Raw Material Economies Among Prehistoric Hunter-Gatherers, Lawrence, University of Kansas Publications, Anthropology, 19, p. 33-48.

1995: «Biache Saint-Vaast, Level IIa: A comparison of analytical approaches", in Dibble H., BAR-Yosef O. (DIR.), The Definition and Interpretation of Levallois Variability, Madison, Prehistory Press, p. 96-116.

1997: «Platform Variability and Flake Morphology: a Comparison of Experimental and Archaeological Data and Implications for Interpreting Prehistoric Lithic Technological Strategies", Lithic Technology, 22, 2, p. 150-170.

Dibble H., BERna F., GOldberg P., MCPherRon S., MENTZER S., NIVEN L., RICHTER D., THÉRY-PARISOT I., SANDGATHE D., TURQ A.

2009: "A Preliminary Report on Pech de l'Azé IV, Layer 8 (Middle Paleolithic, France) ", Paleo-Anthropology, p. 182-219.

Dibble H., Chase P., MCPherron S., TUFFREAU A.

1997: "Testing the Reality of a Living Floor with Archaeological Data», American Antiquity, 62, 4, p. 629-651.

Dibble H., HoldawAY S., LENOIR M., MCPHERRON S., ROTH B., SANDERS-

GRAY H.

1995: "Techniques of Excavation and Analysis», in DIBBLE H., LENOIR M. (DIR.), The Middle Paleolithic Site of Combe-Capelle Bas (France), Philadelphia, University of Pennsylvania Museum, p. 27-40.

\section{DIBBLE H., LENOIR M.}

1995: The Middle Paleolithic Site of Combe-Capelle Bas (France), Philadelphia, University of Pennsylvania Museum, 366 p.

DibBLE H., MCPHERRON S.

1988: «On the Computerization of Archaeological Projects", Journal of Field Archaeology, 15, 4, p. 431-440.
1992: "Computers and Prehistoric Archaeology», Les Cahiers de la vallée de la Couze, 2-3, CIRPC, p. 69-74.

1996: Combe-Capelle on CD-ROM: A Multimedia Companion to The Middle Paleolithic Site of Combe-Capelle Bas (France), Philadelphia, University of Pennsylvania Museum.

1997: "The Making of Combe-Capelle on CD-ROM », Journal of Field Archaeology, 24, 1, p. 59-66.

2001: «Pech de l'Azé IV: bilan scientifique $2000 »$, Service régional d'archéologie Aquitaine, Paris, Ministère de la Culture et de la Communication, p. 11-12.

2006: «The Missing Mousterian», Current Anthropology, 47, 5, p. 777-803.

2007: «Truncated-Faceted Pieces: Hafting Modification, Retouch, or Cores?" in MCPHERRON S. (DIR.), Tools versus Cores Alternative Approaches to Stone Tool Analysis, Newcastle, Cambridge Scholars Publishing, p. 75-90.

\section{Dibble H., McPherron S., Chase P.,} FARRAND W., DEBÉNATH A.

2006: "Taphonomy and the Concept of Paleolithic Cultures: The Case of the Tayacian from Fontéchevade», PaleoAnthropology, p. 1-21.

\section{DibBle H., ROLLAND N.}

1992: «On Assemblage Variability in the Middle Paleolithic of Western Europe: History, Perspectives and a New synthesis», in DibBle H., Mellars P. (DIR.), The Middle Paleolithic: Adaptation, Behavior and Variability, Philadelphia, University Museum Monograph, 78, University Museum Symposium Series, p. 1-28.

Dibble H., RACZEK T. P., MCPHERron S.

2005: «Excavator Bias at the Site of Pech de l'Azé IV (France)», Journal of Field Archaeology, 30, 3, p. 317-328.

\section{FENELON P.}

1951: Le Périgord: étude morphologique, Thèse, Paris, A. Lahure, 526 p.

1974: «Karsts de type tropical sous climat tempéré ", Mémoires et documents du CNRS -II- Phénomènes karstiques, Paris, CNRS Éditions (coll. Nouvelle Série, 15), p. 97-103.

\section{FEREMbach D., LEgouX P., FENART R.,} EMPEREUR-BUISSON R., VLCEK E.

1970: L'Enfant du Pech de l'Azé, Paris, Masson et Cie (coll. Archives de l'Institut de paléontologie humaine, 33), $180 \mathrm{p}$.

\section{GENESTE J.-M.}

1985: Analyse lithique d'industries moustériennes du Périgord: une approche technologique du comportement des groupes humains au Paléolithique moyen, Université de Bordeaux-I, Thèse de $3^{\mathrm{e}}$ cycle, $572 \mathrm{p}$.

\section{GOLDBERG P.}

1979: «Micromorphology of Pech de l'Azé II: sediments", Journal of Archaeological Sciences, 6, p. 17-47.

\section{GOLDBERG P., MACPHAIL R.}

2003: «Strategies and Techniques in Collecting Micromorphology Samples», Geoarchaeology, 18, 5, p. 571-578.

Goldberg P., Nash D., Petraglia M.

1993: Formation Processes in Archaeological Context, Madison, Prehistory Press (coll. Monographs in World Archaeology, 17), $188 \mathrm{p}$.

\section{GRÜN R., MELlaRs P., LAVILle H.}

1991: «ESR Chronology of a 100,000 year Archaeological Sequence at Pech de l'Azé II (France) », Antiquity, 65, p. 544-551.

GRÜN R., YAN G., MCGULlOCH M., MORTIMER G.

1999: «Detailed Mass Spectrometric U-series Analyses of Two Teeth from the Archaeological Site of Pech de l'Azé II: Implications for Uranium Migration and Dating», Journal of Archaeological Science, 26, 10, p. 1301-1310.

HILLS E. S.

1972: Elements of Structural Geology, Londres, Chapman \& Hall (Sciences Paperbacks)/ New York, John Wiley \& Sons, Inc., 502 p.

\section{HILLSON S.}

2002: Dental Anthropology, Cambridge, Cambridge University Press, 373 p.

KARKANAS P., BAR-YOSEF O., GOLDbERG P., WEINER S.

2000: «Diagenesis in Prehistoric Caves: the Use of Minerals that Form in Situ to Assess the Completeness of the Archaeological Record", Journal of Archaeological Science, 27, 10, p. 915-929.

KARKANAS P., KYPARISSI-APOSTOLIKA N., BAR-YOSEF O., WEINER S.

1999: «Mineral Assemblages in Theopetra, Greece: A Framework for Understanding Diagenesis in a Prehistoric Cave ", Journal of Archaeological Science, 26, 9, p. 1171-1180. 


\section{KONIK S.}

1999: Les Dépôts de pente du Périgord noir, distribution et mise en place: origine des matériaux et étapes de l'évolution des versants, Thèse de Doctorat, Université de Paris-1-PanthéonSorbonne, $473 \mathrm{p}$.

LAQUAY G.

1981: Recherches sur les faunes du Würm I en Périgord, Thèse de $3^{\mathrm{e}}$ cycle, Université de Bordeaux-I, 506 p.

LARTet E., CHRisty H.

1864: «Cavernes du Périgord", Revue archéologique, 1, p. 233-267.

LAUTrou A.

1997: Anatomie dentaire, Paris, Masson, 264 p.

\section{LAVILLE H.}

1973a: Climatologie et chronologie du Paléolithique en Périgord: études sédimentologiques et dépôts en grotte et sous abris, Thèse de Doctorat, Université de Bordeaux-I, 720 p.

1973b: «The Relative Position of Mousterian Industries in the Climatic Chronology of the Early Würm in the Périgord", World Archaeology, 4, 3, p. 323-329.

\section{LAVIlle H., RigaUd J.-P., SACKetT J.}

1980: Rock Shelters of the Perigord: Geological Stratigraphy and Archaeological Succession, New York, Academic Press, 371 p.

\section{LEGOUX P.}

1970: «Étude odontologique de l'enfant néandertalien du Pech de l'Azé», in FEREMBACH D., LEgOUX P., FENART R., EMPEREUR-BUISSON R., VLECK E (DIR.), L'Enfant du Pech de l'Azé, Paris, Masson et Cie (coll. Archives de l'Institut de paléontologie humaine, 33), p. 53-87.

\section{LENOBLE A., BERTRAN P.}

2004: «Fabric of Paleolithic Levels: Methods and Implications for Site Formation Processes ", Journal of Archaeological Science, 31, 4, p. 457-469.

\section{MAUREILle B.}

2001: Variabilité dans le genre Homo: les men surations des couronnes dentaires déciduales et permanentes, Synthèse de l'activité scientifique pour l'obtention de l'habilitation à diriger des recherches, Laboratoire d'Anthropologie, Université de Bordeaux-I, 168 p.

\section{MAUREIlle B., SORESSI M.}

2000: «À propos de la position chronostratigraphique de l'enfant du Pech de l’Azé I (commune de Carsac, Dordogne) : la résurrection du fantôme ", Paléo, 12, p. 339-352

\section{MCBrearty S., BiShOP L., PlUMmer T.,} DEWAR R., CONARD N.J.

1998: «Tools Underfoot: Human Trampling as an Agent of Lithic Artifact Edge Modification ", American Antiquity, 63, 1, p. 105129.

\section{MCPHERRON S.}

2005: «Artifact Orientations and Site Formation Processes from Total Station Proveniences", Journal of Archaeological Science, 32, p. 1003-1014.

\section{MCPHERRON S., DibBle H.}

1987: «Hardware and Software Complexity in Computerizing Archaeological Projects ", Advances in Computer Archaeology, 4, p. $25-40$.

2000: "The Lithic Assemblages of Pech de l'Azé IV (Dordogne, France) ", Préhistoire européenne, 15, p. 9-43.

2002: Using Computers in Archaeology: A Practical Guide, Boston, McGraw-Hill Mayfield, $254 \mathrm{p}$.

MCPHerRon S., Soressi M., Dibble H.

2001: «Deux nouveaux projets de recherche à Pech de l'Azé (Dordogne, France)", Préhistoire du Sud-Ouest, 8, p. 11-30.

MCPherron S., Dibble H., Goldberg P. 2005: «Z», Geoarchaeology, 20, 3, p. 243-262.

MEIGNen L., BAR-YoSEF O., GOLdBERG P.

1989: "Les structures de combustion moustériennes de la grotte de Kébara (Mont Carmel, Israël) », in OLIVE M., TABORINY. (DIR.), Nature et fonction des foyers préhistoriques, Actes du colloque international de Nemours, 12-14 mai 1987, APRAIF (coll. Mémoires du musée de Préhistoire d'Îlede-France, 2), p. 141-146.

2007: "The Hearths at Kebara Cave and their Role in Site Formation Processes ", in BARYosef O., MEIGNEN L. (DIR.), The Middle and Upper Paleolithic Archaeology of the Kebara Cave, Mt Carmel, Israel, Cambridge, Harvard University, Peabody Museum of Archaeology and Ethnology (American School of Prehistoric Research-Bulletin, $49)$, p. 91-122.

\section{MELLARS P.}

1969: "The Chronology of Mousterian Industries in the Périgord Region of South-West France", Proceedings of the Prehistoric Society, 35, p. 134-171.

1988: «The Chronology of the SouthWest French Mousterian: A Review of the Current Debate", in BINFORD L., RIGAUD J.-P. (DIR.), L'Homme de Neandertal -4- La technique, Actes du colloque international de Liège, 4-7 déc. 1986, Liège, Université de Liège (coll. ERAUL, 31)p. 97-120.

1996: The Neanderthal Legacy: An Archaeological Perspective from Western Europe, Princeton, Princeton University Press, 472 p.

\section{MELLARS P., GRÜN R.}

1991: «A Comparison of the Electron Spin Resonance and Thermoluminescence Dating Methods: the Results of ESR Dating at Le Moustier (France) », Cambridge Archaeological Journal, 1, 2, p. 269-276.

MoOrRees C. F. A., FANNing E. A., HUNT E. E.

1963: «Age Variation of Formation Stages for Ten Permanent Teeth", Journal of Dental Research, 42, 6, p. 1490-1502.

\section{Morala A.}

1983: «À propos des matières premières lithiques en Haut-Agenais », BSPF, 80, 6, p. 169.

\section{ORTOLI T.}

2004: A Geological Interpretation to an Archaeological Problem: Micromorphology and Site History at Pech de l'Azé IV, Dordogne Valley, France, MA Thesis, Boston University, Department of Archaeology.

PATTE É.

1955: Les Néandertaliens: anatomie, physiologie, comparaisons, Paris, Masson, 559 p.

1957: L'Enfant néanderthalien du Pech de l'Azé, Paris, Masson, 230 p.

\section{Platel J.-P.}

1989: Le Crétacé supérieur de la plate-forme septentrionale du bassin d'Aquitaine: stratigraphie et évolution géodynamique, Orléans, documents du BRGM, 164, 573 p.

RIGAUD J.-P., SIMEK J.-F., GE TH.

1995: «Mousterian Fires from Grotte XVI (Dordogne, France) », Antiquity, 69, 266, p. 902-912.

ROLlAND N., DibBle H.

1990: «A New Synthesis of Middle Paleolithic Variability", American Antiquity, 55, 3, p. $480-499$.

\section{ROTH B., DibBle H.}

1998: "The Production and Transport of Blanks and Tools at the French Middle Paleolithic Site of Combe-Capelle Bas", American Antiquity, 63, 1, p. 47-62. 
SALOMON J.-N., ASTRUC J.-G.

1992: «Exemple en zone tempérée d'un paléocryptokarst tropical exhumé (la cuvette du Sarladais, Dordogne)", in SALOMON J.-N., MAIRE R. (DIR.), Karsts et évolutions climatiques, Talence, Presses universitaires de Bordeaux, p. 432-447.

\section{SANDGATHE D. M.}

2005: An Analysis of the Levallois Reduction Strategy Using a Design Theory Framework, Thesis, Department of Archaeology, Simon Fraser University.

\section{SCHIEGL S., GOLDBERG P.,} PFRETZSCHNeR H.-U., CONARd N.J.

2003: «Paleolithic Burnt Bone Horizons from the Swabian Jura: Distinguishing between in situ Fireplaces and Dumping Areas", Geoarchaeology, 18, 5, p. 541-565.

SCHWARCZ H. P., BLACKWELL B.

1983: «230Th/234U Age of a Mousterian Site in France», Nature, 301, p. 236-237.

SÉRONIE-VIVIEN M.-R., SÉRONIE-VIVIEN M. 1987: Les Silex du Mésozoïque nord-aquitain: approche géologique de l'étude du silex pour servir à la recherche, Bordeaux (coll. Bulletin de la Société linnéenne de Bordeaux, 15), $135 \mathrm{p}$.

\section{SORESSI M.}

2002: Le Moustérien de tradition acheuléenne $d u$ sud-ouest de la France: discussion sur la signification du faciès à partir de l'étude comparée de quatre sites: Pech de l'Azé I, le Moustier, la Rochette et la grotte XVI, Thèse de Doctorat, Université de Bordeaux-I, $330 \mathrm{p}$.

\section{SORESSI M., ARMAND D., D'ERRICO F.,} PUBERT E., JONES H., RINK J., TEXIER J.-P., VIVENT D.

2002: «Pech de l'Azé I (Carsac): nouveaux travaux sur le Moustérien de tradition acheuléenne», $B S P F, 99,1$, p. 5-11.

\section{SMITH B. H.}

1984: «Patterns of Molar Wear in HunterGatherers and Agriculturalists", American Journal of Physical Anthropology, 63, p. 39-56.

SORESSI M., RENDU W., TEXIER J.-P., Claud E., DaUlny L., D'ERrico F., LaRoulandie V., MaUREILle B., Niclot M., SCHWORTZ S., TILliER A.-M.

2008: «Pech de l'Azé I (Dordogne, France): nouveau regard sur un gisement moustérien de tradition acheuléenne connue depuis le $\mathrm{xix}^{\mathrm{e}}$ siècle", in JAUBERT J., BORDES J.-G., ORTEGA I. (DIR.), Les Sociétés du Paléolithique dans le grand sudouest de la France: nouveaux gisements, nouveaux résultats, nouvelles méthodes, Actes des journées SPF, Université de Bordeaux-I, 24-25 nov. 2006, SPF (coll. Mémoires de la SPF, 47), p. 95-132.

\section{STOOPS G.}

2003: Guidelines for Analysis and Description of Soil and Regolith Thin Sections, Madison WI, Soil Science Society of America, 184 p.

\section{TEXIER J.-P.}

2000: «A propos des processus de formation des sites préhistoriques », Paléo, 12, p. 379386 .

2006: «Nouvelle lecture géologique du site paléolithique du Pech de l'Azé II (Dordogne, France) ", Paléo, 18, p. 217-235.

TURNER GH. G., NICHOL GH. R., SCOTT G. R.

1991: «Scoring Procedures for Key Morphological Traits of the Permanent Dentition: the Arizona State University Dental Anthropology System ", in KELLEY M. A., SPENSER LARSEN C. (DIR.), Advances in Dental Anthropology, New York, Wiley-Liss, p. 13-31.

\section{TURQ A.}

2000: Le Paléolithique inférieur et moyen entre les vallées de la Dordogne et du Lot (coll. Paléo, Suppl. 2), 456 p.
Turo A., ANTignac G., Roussel P.

1999: «Les silicifications coniaciennes du Sarladais et du Gourdonnais: inventaire et implications archéologiques ", Paléo, 11, p. $145-160$.

TURQ A., Dibble H., FAIVRe J.P., GOLDBERG P., MCPHERRON S.P., SANDGATHE D.

2008: «Le Moustérien du Périgord noir: quoi de neuf ?» in JAUBERT J., BORDES J.-G., Ortega I. (DIR.), Les Sociétés du Paléolithique dans le grand sud-ouest de la France: nouveaux gisements, nouveaux résultats, nouvelles méthodes, Actes des journées SPF, Université de Bordeaux-I, 24-25 nov. 2006, SPF (coll. Mémoires de la SPF, 47), p. 83-93.

UBELAKER D. $\mathbf{H}$.

1984: Human Skeletal Remains: Excavation, Analysis, Interpretation, Washington, Taraxacum, $119 \mathrm{p}$.

\section{VANDERMEERSCH B.}

1995: "L'homme: étude d'une incisive déciduale", in Defleur A., CREgutBONNOURE E. (DIR.), Le Gisement paléolithique moyen de la grotte des Cèdres (le Plan-d'Aups, Var), Paris, éd. de la Maison des sciences de l'homme (coll. DAF, 49), p. 49-52.

\section{VAUFREY R.}

1933: «Le Moustérien de tradition acheuléenne du Pech de l'Azé (Dordogne)", L'Anthropologie, 93, p. 125-127.

\section{WATSON G. S.}

1965: «Equatorial Distributions on a Sphere», Biometrika, 52, 1-2, p. 193-201.

\section{WOODCOCK N. H.}

1977: «Specification of Fabric Shapes Using an Eigen Value Method», Geological Society of America Bulletin, 88, 9, p. 1231-1236. 